\title{
Chiral Brønsted Acid Catalyzed Enantioselective Phosphonylation of Allylamine via Oxidative Dehydrogenation Coupling
}

Ming-Xing Cheng, $\uparrow$ Ran-Song Ma, $\uparrow$ Qiang Yang, $\uparrow$ Shang-Dong Yang*†t

$\dagger$ State Key Laboratory of Applied Organic Chemistry, Lanzhou University, Lanzhou 730000, P. R. China.

¥State Key Laboratory for Oxo Synthesis and Selective Oxidation, Lanzhou Institute of Chemical Physics, Chinese Academy of Sciences, Lanzhou, 730000, P. R. China.

\section{Supporting Information}

I . General Methods and Materials .........................................

II. Typical Procedures for the Synthesis of Chiral Phosphoric Acid .........S-2

III. Typical Procedures for the Synthesis of Substrates .......................S-3

IV. Detailed Reaction Conditions Optimization ................................S-3

V. General Procedure for the Enantioselective Synthesis of $\alpha$-amino Phosphonates .....................................................5-5

VI. Analytical Data of Products ..................................................5-5

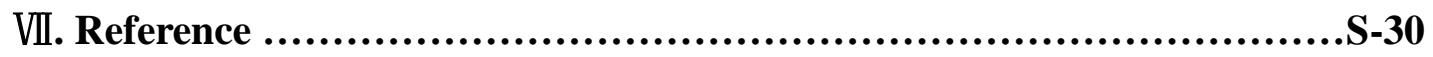

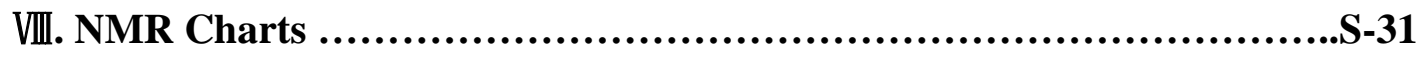




\section{I . General Methods and Materials}

${ }^{1} \mathrm{H}$ and ${ }^{13} \mathrm{C}$ NMR spectra were recorded on a Bruker advance III 400 spectrometer $(400 \mathrm{MHz}$ for ${ }^{1} \mathrm{H}$ and $100 \mathrm{MHz}$ for ${ }^{13} \mathrm{C}$ ) in $\mathrm{CDCl}_{3}$ with TMS as internal standard. Chemical shifts $(\delta)$ were measured in ppm relative to TMS $\delta=0$ for ${ }^{1} \mathrm{H}$, or to chloroform $\delta=77.0$ for ${ }^{13} \mathrm{C}$ as internal standard. ${ }^{31} \mathrm{P}$ NMR spectra and ${ }^{19} \mathrm{~F}$ NMR were recorded on the same instrument. Data are reportedas follows: Chemical shift, multiplicity $(\mathrm{s}=$ singlet, $\mathrm{d}=$ doublet, $\mathrm{t}=$ triplet, $\mathrm{q}=$ quartet, $\mathrm{m}$ $=$ multiplet), Coupling constants, J, are reported in hertz. Mass data were measured with Thermo Scientific DSQ II mass spectrometer. The starting materials were purchased from Aldrich, Acros Organics, J\&K Chemicals Adamas-beta or TCI and used without further purification. Solvents were dried and purified according to the procedure from "Purification of Laboratory Chemicals book". Thin-layer chromatography (TLC) was performed using 60 mesh silica gel plates visualized with short-wavelength UV light $(254 \mathrm{~nm})$. . Enantiomeric excess is determined by HPLC analysis (Agilent 1260).

\section{Typical Procedures for the Synthesis of Chiral Phosphoric Acid}

$1 \mathrm{a}-1 \mathrm{k}$ are prepared based on the procedure reported by Wipf et al. ${ }^{1 \mathrm{a}} 1 \mathrm{l}$ is prepared based on the procedure reported by Terada et al. ${ }^{1 \mathrm{~b}} \mathbf{1 0 - 1 p}$ are prepared based on the procedure reported by Toste et al. ${ }^{1 \mathrm{c}} \mathbf{1 m - 1} \mathbf{n}, \mathbf{1 q - 1}$ s were purchased from DAICEL.

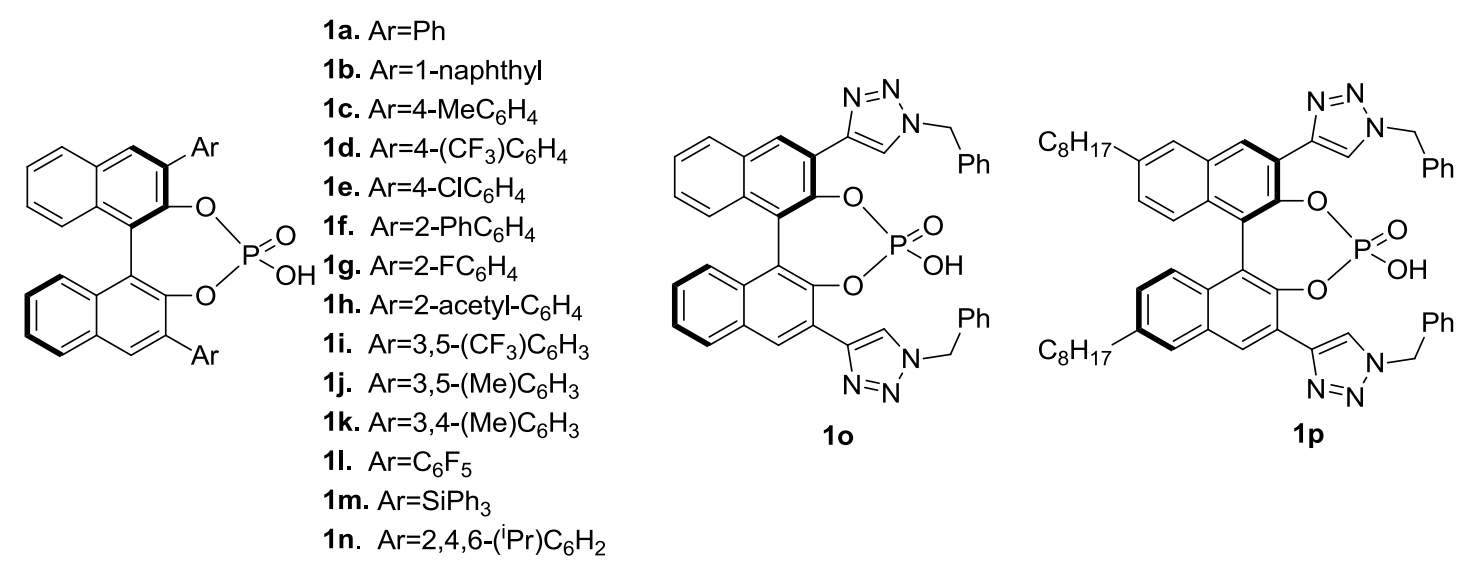<smiles>O=P(O)(Oc1cccc2c(-c3ccccc3)cccc12)Oc1c(-c2ccccc2)cc(-c2ccccc2)c2ccccc12</smiles>

$1 q$<smiles></smiles>

$1 \mathrm{r}$<smiles>O=P(O)(O)OC1=CC2=CC=CC(=CC=C3C=CC4=CC=CC2=C(c2ccccc2)C2=C5C=Cc6ccccc6C=C5CC(=C2OP(=O)(O)O3)OP(=O)(O)O4)C=C1</smiles>

$1 \mathrm{~s}$ 


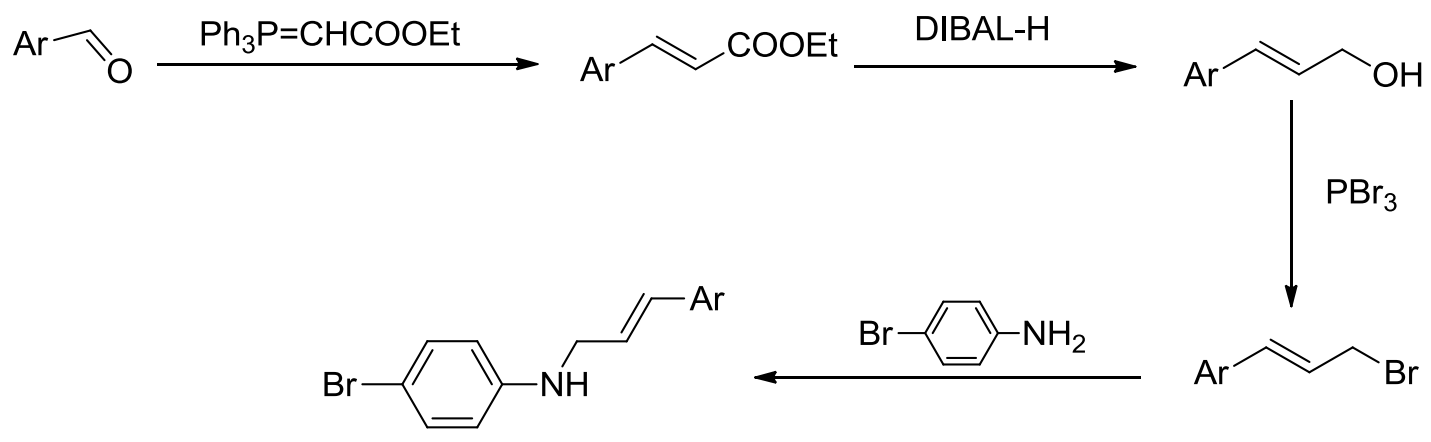

\section{Detailed Reaction Conditions Optimization}

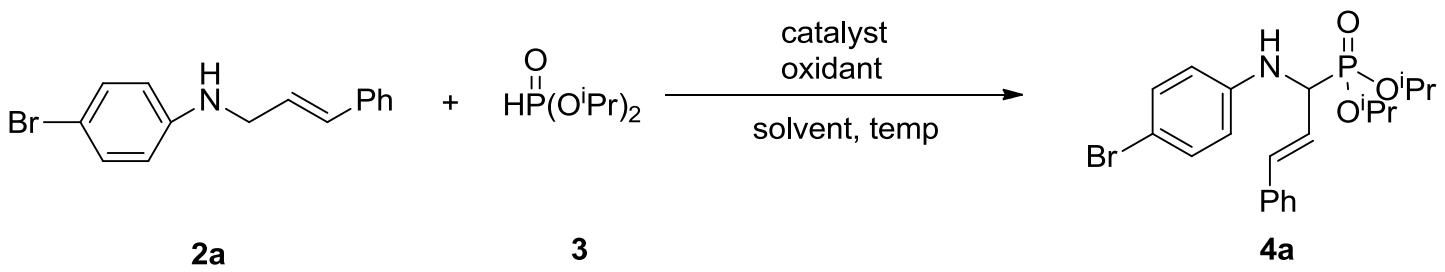

Table S1 Screening Different Catalysts ${ }^{\mathrm{a}}$

\begin{tabular}{|c|c|c|c|c|c|c|}
\hline Entry & Cat. (10 mol\%) & Oxidant (1.1 equiv) & $\mathrm{T}\left({ }^{\circ} \mathrm{C}\right)$ & Solvent & Yield $(\%)^{\mathrm{b}}$ & $\operatorname{Ee}(\%)^{\mathrm{c}}$ \\
\hline 1 & 1a & $\mathrm{Ag}_{2} \mathrm{CO}_{3}$ & 50 & Toluene & 27 & 42 \\
\hline 2 & $1 \mathrm{~b}$ & $\mathrm{Ag}_{2} \mathrm{CO}_{3}$ & 50 & Toluene & 31 & 63 \\
\hline 3 & $1 \mathrm{c}$ & $\mathrm{Ag}_{2} \mathrm{CO}_{3}$ & 50 & Toluene & 11 & 43 \\
\hline 4 & 1d & $\mathrm{Ag}_{2} \mathrm{CO}_{3}$ & 50 & Toluene & 31 & 53 \\
\hline 5 & $1 \mathrm{e}$ & $\mathrm{Ag}_{2} \mathrm{CO}_{3}$ & 50 & Toluene & 40 & 50 \\
\hline 6 & 1f & $\mathrm{Ag}_{2} \mathrm{CO}_{3}$ & 50 & Toluene & 27 & 58 \\
\hline 7 & $1 \mathrm{~g}$ & $\mathrm{Ag}_{2} \mathrm{CO}_{3}$ & 50 & Toluene & 24 & 58 \\
\hline 8 & $1 \mathrm{~h}$ & $\mathrm{Ag}_{2} \mathrm{CO}_{3}$ & 50 & Toluene & 20 & 64 \\
\hline 9 & $1 \mathbf{i}$ & $\mathrm{Ag}_{2} \mathrm{CO}_{3}$ & 50 & Toluene & 49 & 62 \\
\hline 10 & $1 \mathrm{j}$ & $\mathrm{Ag}_{2} \mathrm{CO}_{3}$ & 50 & Toluene & 55 & 57 \\
\hline 11 & $1 \mathrm{k}$ & $\mathrm{Ag}_{2} \mathrm{CO}_{3}$ & 50 & Toluene & 16 & 48 \\
\hline 12 & 11 & $\mathrm{Ag}_{2} \mathrm{CO}_{3}$ & 50 & Toluene & 21 & 82 \\
\hline 13 & $1 \mathrm{~m}$ & $\mathrm{Ag}_{2} \mathrm{CO}_{3}$ & 50 & Toluene & 20 & race \\
\hline 14 & 1n & $\mathrm{Ag}_{2} \mathrm{CO}_{3}$ & 50 & Toluene & trace & 10 \\
\hline 15 & 10 & $\mathrm{Ag}_{2} \mathrm{CO}_{3}$ & 50 & Toluene & N.R & \\
\hline 16 & $1 p$ & $\mathrm{Ag}_{2} \mathrm{CO}_{3}$ & 50 & Toluene & N.R & \\
\hline 17 & $1 q$ & $\mathrm{Ag}_{2} \mathrm{CO}_{3}$ & 50 & Toluene & 31 & 8 \\
\hline 18 & $1 \mathbf{r}$ & $\mathrm{Ag}_{2} \mathrm{CO}_{3}$ & 50 & Toluene & 16 & 61 \\
\hline 19 & 1s & $\mathrm{Ag}_{2} \mathrm{CO}_{3}$ & 50 & Toluene & trace & race \\
\hline
\end{tabular}

[a] All reactions were carried out in the presence of $0.1 \mathrm{mmol}$ of $\mathbf{2 a}$ and $0.2 \mathrm{mmol}$ of $\mathbf{3}$ in $2.0 \mathrm{~mL}$ solvent under Ar atmosphere for $72 \mathrm{~h} ; \mathrm{N} . \mathrm{R}=$ no reaction. [b] Yield of isolated product. [c] The ee was determined by HPLC analysis using chiral columns. 
Table S2 Screening Different Oxidant ${ }^{\mathrm{a}}$

\begin{tabular}{|c|c|c|c|c|c|c|}
\hline Entry & Cat. (10 mol\%) & Oxidant (1.1 equiv) & $\mathrm{T}\left({ }^{\circ} \mathrm{C}\right)$ & Solvent & Yield $(\%)^{\mathrm{b}}$ & $\operatorname{Ee}(\%)^{\mathrm{c}}$ \\
\hline 1 & 11 & DDQ & 50 & Toluene & 85 & 63 \\
\hline 2 & 11 & BQ & 50 & Toluene & 60 & 67 \\
\hline 3 & 11 & $\mathrm{~K}_{2} \mathrm{~S}_{2} \mathrm{O}_{8}$ & 50 & Toluene & 71 & 50 \\
\hline 4 & 11 & Oxone & 50 & Toluene & 58 & 55 \\
\hline 5 & 11 & $\mathrm{PhI}(\mathrm{OAc})_{2}$ & 50 & Toluene & 47 & 56 \\
\hline 6 & 11 & DICP & 50 & Toluene & trace & \\
\hline 7 & 11 & IBX & 50 & Toluene & 62 & 68 \\
\hline 8 & 11 & $\mathrm{O}_{2}$ & 50 & Toluene & trace & \\
\hline 9 & 11 & $\mathrm{AgOAc}$ & 50 & Toluene & 19 & 80 \\
\hline 10 & 11 & $\mathrm{Ag}_{2} \mathrm{O}$ & 50 & Toluene & 34 & 82 \\
\hline 11 & 11 & $\mathrm{AgClO}_{4}$ & 50 & Toluene & trace & \\
\hline 12 & 11 & $\mathrm{AgNO}_{2}$ & 50 & Toluene & trace & \\
\hline 13 & 11 & $\mathrm{Ag}_{3} \mathrm{PO}_{4}$ & 50 & Toluene & 6 & 80 \\
\hline 14 & 11 & $\mathrm{AgCl}$ & 50 & Toluene & 23 & 75 \\
\hline
\end{tabular}

[a] All reactions were carried out in the presence of $0.1 \mathrm{mmol}$ of $\mathbf{2 a}$ and $0.2 \mathrm{mmol}$ of $\mathbf{3}$ in $2.0 \mathrm{~mL}$ solvent under $\mathrm{Ar}$ atmosphere for $72 \mathrm{~h} ; \mathrm{N} . \mathrm{R}=$ no reaction. [b] Yield of isolated product. [c] The ee was determined by HPLC analysis using chiral columns.

Table S3 Screening Different Solvent ${ }^{\mathrm{a}}$

\begin{tabular}{|c|c|c|c|c|c|c|}
\hline Entry & Cat. $(10 \mathrm{~mol} \%)$ & Oxidant $(1.1$ equiv $)$ & $\mathrm{T}\left({ }^{\circ} \mathrm{C}\right)$ & Solvent & Yield $(\%)^{\mathrm{b}}$ & Ee $(\%)^{\mathrm{c}}$ \\
\hline 1 & $\mathbf{1 1}$ & $\mathrm{Ag}_{2} \mathrm{CO}_{3}$ & 50 & $\mathrm{DCM}$ & 67 & 74 \\
\hline 2 & $\mathbf{1 1}$ & $\mathrm{Ag}_{2} \mathrm{CO}_{3}$ & 50 & DCE & 58 & 73 \\
\hline 3 & $\mathbf{1 1}$ & $\mathrm{Ag}_{2} \mathrm{CO}_{3}$ & 50 & Dioxane & 33 & 78 \\
\hline 4 & $\mathbf{1 1}$ & $\mathrm{Ag}_{2} \mathrm{CO}_{3}$ & 50 & $\mathrm{CH}_{3} \mathrm{CN}$ & $\mathrm{N} . \mathrm{R}$ & \\
\hline 5 & $\mathbf{1 1}$ & $\mathrm{Ag}_{2} \mathrm{CO}_{3}$ & 50 & $\mathrm{CH}_{3} \mathrm{NO}_{2}$ & 20 & 73 \\
\hline 6 & $\mathbf{1 1}$ & $\mathrm{Ag}_{2} \mathrm{CO}_{3}$ & 50 & Toluene & 21 & 82 \\
\hline 7 & $\mathbf{1 1}$ & $\mathrm{Ag}_{2} \mathrm{CO}_{3}$ & 50 & o-xylene & 21 & 81 \\
\hline 8 & $\mathbf{1 1}$ & $\mathrm{Ag}_{2} \mathrm{CO}_{3}$ & 50 & m-xylene & 22 & 84 \\
\hline 9 & $\mathbf{1 1}$ & $\mathrm{Ag}_{2} \mathrm{CO}_{3}$ & 50 & p-xylene & 20 & 82 \\
\hline 10 & $\mathbf{1 1}$ & $\mathrm{Ag}_{2} \mathrm{CO}_{3}$ & 50 & xylene & 25 & 81 \\
\hline 11 & $\mathbf{1 1}$ & $\mathrm{Ag}_{2} \mathrm{CO}_{3}$ & 50 & PhCl & 82 & 80 \\
\hline 12 & $\mathbf{1 1}$ & $\mathrm{Ag}_{2} \mathrm{CO}_{3}$ & 50 & 1,3 -PhCl & 50 & 80 \\
\hline 13 & $\mathbf{1 1}$ & $\mathrm{Ag}_{2} \mathrm{CO}_{3}$ & 50 & Mesitylen & 26 & 82 \\
\hline 14 & $\mathbf{1 1}$ & $\mathrm{Ag}_{2} \mathrm{CO}_{3}$ & 50 & TICP & 48 & 80 \\
\hline 15 & $\mathbf{1 1}$ & $\mathrm{Ag}_{2} \mathrm{CO}_{3}$ & 50 & Anisole & 36 & 82 \\
\hline
\end{tabular}

[a] All reactions were carried out in the presence of $0.1 \mathrm{mmol}$ of $\mathbf{2 a}$ and $0.2 \mathrm{mmol}$ of $\mathbf{3}$ in $2.0 \mathrm{~mL}$ solvent under $\mathrm{Ar}$ atmosphere for $72 \mathrm{~h}$; TICP $=1,3,5$-Triisopropylbenzene; N.R = no reaction. [b] Yield of isolated product. [c] The ee was determined by HPLC analysis using chiral columns. 
Table S4 Screening the Loading of Oxidant and Solvent ${ }^{\mathrm{a}}$

\begin{tabular}{|c|c|c|c|c|c|c|}
\hline Entry & Cat. $(10 \mathrm{~mol} \%)$ & Oxidant & $\mathrm{T}\left({ }^{\circ} \mathrm{C}\right)$ & Solvent & Yield $(\%)^{\mathbf{b}}$ & Ee $(\%)^{\mathbf{c}}$ \\
\hline 1 & $\mathbf{1 l}$ & $\mathrm{Ag}_{2} \mathrm{CO}_{3}(1.0$ equiv $)$ & 50 & $2.0 \mathrm{~mL}$ & 22 & 84 \\
\hline 2 & $\mathbf{1 l}$ & $\mathrm{Ag}_{2} \mathrm{CO}_{3}(1.2$ equiv $)$ & 50 & $2.0 \mathrm{~mL}$ & 18 & 84 \\
\hline 3 & $\mathbf{1 l}$ & $\mathrm{Ag}_{2} \mathrm{CO}_{3}(1.5$ equiv $)$ & 50 & $2.0 \mathrm{~mL}$ & 22 & 84 \\
\hline 4 & $\mathbf{1 l}$ & $\mathrm{Ag}_{2} \mathrm{CO}_{3}(2.0$ equiv $)$ & 50 & $2.0 \mathrm{~mL}$ & 18 & 84 \\
\hline 5 & $\mathbf{1 l}$ & $\mathrm{Ag}_{2} \mathrm{CO}_{3}(1.1$ equiv $)$ & 50 & $0.5 \mathrm{~mL}$ & 50 & 70 \\
\hline 6 & $\mathbf{1 l}$ & $\mathrm{Ag}_{2} \mathrm{CO}_{3}(1.1$ equiv $)$ & 50 & $1.0 \mathrm{~mL}$ & 32 & 80 \\
\hline 7 & $\mathbf{1 l}$ & $\mathrm{Ag}_{2} \mathrm{CO}_{3}(1.1$ equiv $)$ & 50 & $1.5 \mathrm{~mL}$ & 27 & 84 \\
\hline 8 & $\mathbf{1 l}$ & $\mathrm{Ag}_{2} \mathrm{CO}_{3}(1.1$ equiv $)$ & 50 & $2.0 \mathrm{~mL}$ & 20 & 84 \\
\hline
\end{tabular}

[a] All reactions were carried out in the presence of $0.1 \mathrm{mmol}$ of $\mathbf{2 a}$ and $0.2 \mathrm{mmol}$ of $\mathbf{3}$ in $\mathrm{m}$-xylene under Ar atmosphere for $72 \mathrm{~h}$. [b] Yield of isolated product. [c] The ee was determined by HPLC analysis using chiral columns.

Table S5 Screening for Reaction Conditions ${ }^{\mathrm{a}}$

\begin{tabular}{|c|c|c|c|c|c|c|}
\hline Entry & Cat. $(10 \mathrm{~mol} \%)$ & Oxidant & Ratio & $\mathrm{T}\left({ }^{\circ} \mathrm{C}\right)$ & Yield $(\%)^{\mathbf{b}}$ & Ee $(\%)^{\mathbf{c}}$ \\
\hline 1 & $\mathbf{1 l}$ & $\mathrm{Ag}_{2} \mathrm{CO}_{3}(1.1$ equiv $)$ & $\mathbf{2 a : 3 = 1 : 2}$ & 50 & 76 & 81 \\
\hline 2 & $\mathbf{1 l}$ & $\mathrm{Ag}_{2} \mathrm{CO}_{3}(1.1$ equiv $)$ & $\mathbf{2 a : 3 = 1 : 1 . 5}$ & 50 & 83 & 83 \\
\hline 3 & $\mathbf{1 l}$ & $\mathrm{Ag}_{2} \mathrm{CO}_{3}(1.1$ equiv $)$ & $\mathbf{2 a : 3 = 1 : 1 . 2}$ & 50 & 84 & 83 \\
\hline 4 & $\mathbf{1 l}$ & $\mathrm{Ag}_{2} \mathrm{CO}_{3}(1.1$ equiv $)$ & $\mathbf{2 a : 3 = 1 : 1}$ & 50 & 88 & 84 \\
\hline 5 & $\mathbf{1 l}$ & $\mathrm{Ag}_{2} \mathrm{CO}_{3}(1.1$ equiv $)$ & $\mathbf{2 a : 3 = 1 : 2}$ & 40 & 29 & 83 \\
\hline 6 & $\mathbf{1 l}$ & $\mathrm{Ag}_{2} \mathrm{CO}_{3}(1.1$ equiv $)$ & $\mathbf{2 a : 3 = 1 : 2}$ & 60 & 54 & 80 \\
\hline
\end{tabular}

[a] All reactions were carried out in the presence of $0.1 \mathrm{mmol}$ of $\mathbf{2 a}$ and $0.2 \mathrm{mmol}$ of $\mathbf{3}$ in $1.5 \mathrm{~mL}$ m-xylene under Ar atmosphere for $168 \mathrm{~h}$. [b] Yield of isolated product. [c] The ee was determined by HPLC analysis using chiral columns.

\section{V . General Procedure for the Enantioselective Synthesis of $\alpha$-aminoPhosphonates}

To a Schlenk tube were added $\mathbf{2 a}(0.1 \mathrm{mmol}), \mathrm{Ag}_{2} \mathrm{CO}_{3}(0.11 \mathrm{mmol}, 1.1$ equiv) and $\mathbf{1 l}(0.01$ $\mathrm{mmol}, 10 \% \mathrm{mmol})$ and charged with argon for three times. Anhydrous $\mathrm{m}$-xylene $(1.5 \mathrm{~mL})$ and $\mathbf{3}$ $(0.1 \mathrm{mmol})$ were added and the mixture was stirred at $50{ }^{\circ} \mathrm{C}$ under Ar for $168 \mathrm{~h}$. After completion of the reaction as monitored by TLC, sat. $\mathrm{NaHCO}_{3}$ solution was added to the mixture to stop the reaction. The mixture was extracted with EtOAc $(3 \times 10 \mathrm{~mL})$ and the combined organic layers were washed with brine, dried over anhydrous $\mathrm{Na}_{2} \mathrm{SO}_{4}$, and concentrated to dryness. The residue is purified by column chromatography on silica gel using n-pentane/EtOAc (4:1) as the eluent to give $\alpha$-amino phosphonate $\mathbf{4 a}$ (40 $\mathrm{mg}, 0.088 \mathrm{mmol}$ ) in $84 \%$ ee.

\section{Analytical Data of Products}<smiles>O=[PH](O)[C@H](/C=C/c1ccccc1)Nc1ccc(Br)cc1</smiles> 


\section{(R, E)-diisopropyl (1-((4-bromophenyl)amino)-3-phenylallyl)phosphonate}

4a: $88 \%$ yield (40 mg, n-pentane/EtOAc $=4: 1$ as the eluent). ${ }^{\mathbf{1}} \mathbf{H} \mathbf{~ N M R}(\mathbf{4 0 0} \mathbf{~ M H z}, \mathbf{C D C l}) \delta 7.34(\mathrm{~d}$, $J=7.3 \mathrm{~Hz}, 2 \mathrm{H}), 7.29$ (t, $J=7.4 \mathrm{~Hz}, 2 \mathrm{H}), 7.26-7.19(\mathrm{~m}, 3 \mathrm{H}), 6.65$ (dd, $J=16.0,4.7 \mathrm{~Hz}, 1 \mathrm{H}), 6.57$ $(\mathrm{d}, J=8.8 \mathrm{~Hz}, 2 \mathrm{H}), 6.24(\mathrm{dt}, J=15.9,5.3 \mathrm{~Hz}, 1 \mathrm{H}), 4.87-4.63(\mathrm{~m}, 2 \mathrm{H}), 4.33(\mathrm{dd}, J=26.4,5.4 \mathrm{~Hz}$, $2 \mathrm{H}), 1.34(\mathrm{dd}, J=6.2,2.9 \mathrm{~Hz}, 6 \mathrm{H}), 1.26(\mathrm{dd}, J=9.7,6.2 \mathrm{~Hz}, 6 \mathrm{H}) .{ }^{\mathbf{1 3}} \mathbf{C} \mathbf{N M R}\left(\mathbf{1 0 1} \mathbf{~ M H z}, \mathbf{C D C l}_{\mathbf{3}}\right) \delta$ $145.72(\mathrm{~d}, J=12.7 \mathrm{~Hz}), 136.15$ (d, $J=3.1 \mathrm{~Hz}), 132.75$ (d, $J=12.3 \mathrm{~Hz}), 131.89,128.51,127.79,126.45$ $(\mathrm{d}, J=1.8 \mathrm{~Hz}), 123.23(\mathrm{~d}, J=4.8 \mathrm{~Hz}), 115.24,109.94,72.07$ (d, $J=7.3 \mathrm{~Hz}), 71.75(\mathrm{~d}, J=7.4 \mathrm{~Hz})$, $54.36(\mathrm{~d}, J=154.3 \mathrm{~Hz}), 24.17(\mathrm{~d}, J=3.3 \mathrm{~Hz}), 24.09(\mathrm{~d}, J=3.4 \mathrm{~Hz}), 23.84(\mathrm{~d}, J=5.3 \mathrm{~Hz}), 23.78(\mathrm{~d}, J=$ $5.2 \mathrm{~Hz}) .{ }^{31} \mathbf{P}$ NMR (162 MHz, $\left.\mathbf{C D C l}_{3}\right) \delta$ 19.97. Enantiomeric excess is 84\% determined by HPLC analysis: Chiralcel $\mathrm{AD}-\mathrm{H}$ (hexane/iPrOH $=50 / 50$, flow rate $=0.5 \mathrm{~mL} / \mathrm{min}, 240.4 \mathrm{~nm}$ ), $\mathrm{t}_{\mathrm{r}}$ (major) = $13.955 \mathrm{~min}, \mathrm{t}_{\mathrm{r}}$ (minor) $=19.294 \mathrm{~min} .[\alpha]_{\mathrm{D}}^{22.2}+96\left(\mathrm{c}=1.0, \mathrm{CHCl}_{3}\right)(84 \%$ ee). HRMS (ESI) for $\mathrm{C}_{21} \mathrm{H}_{27} \mathrm{BrNO}_{3} \mathrm{PNa}\left([\mathrm{M}+\mathrm{Na}]^{+}\right)$: calcd 474.0804, found 474.0811.
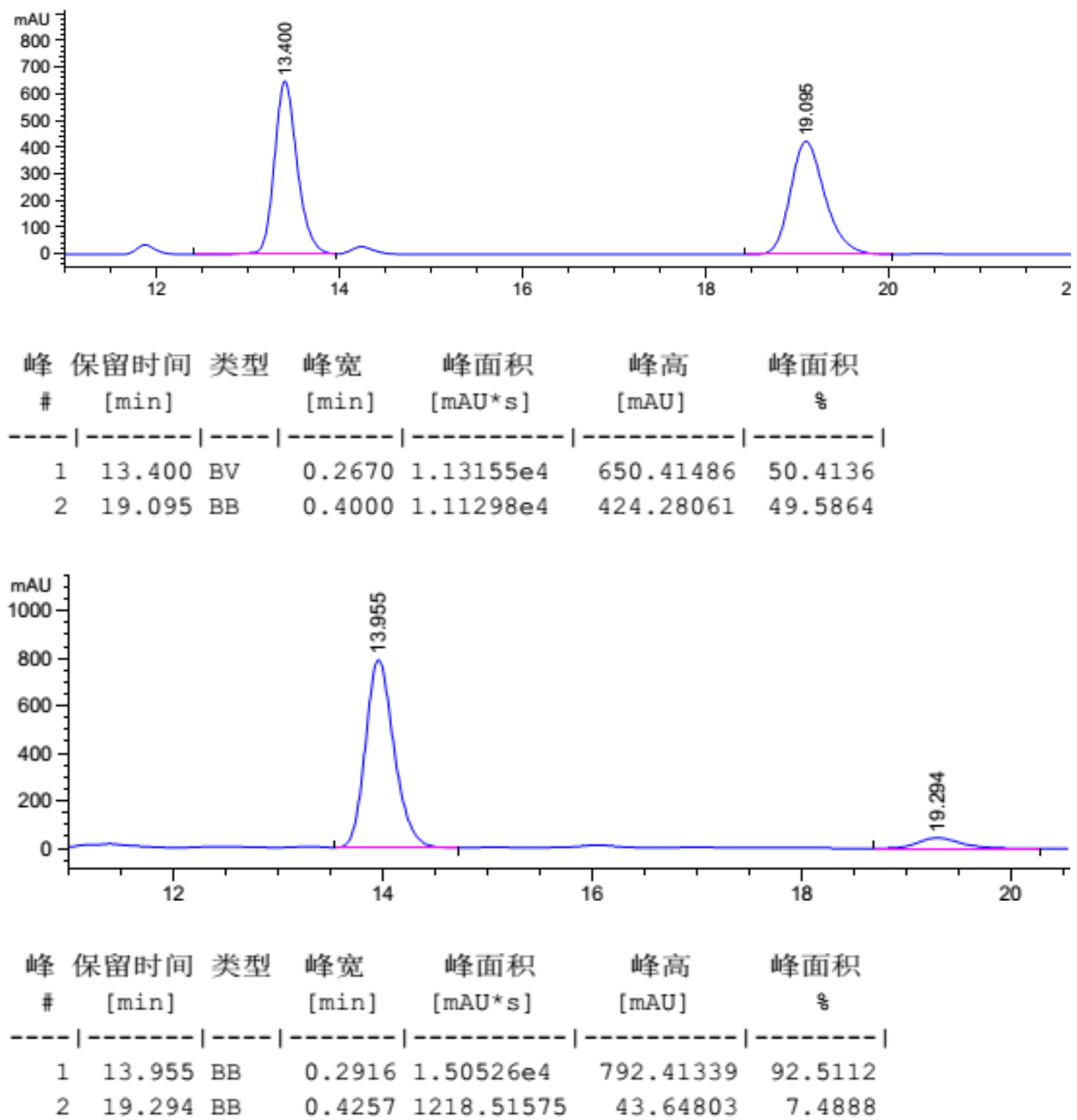
<smiles>COc1ccc(N[C@@H](/C=C/c2ccccc2)P(=O)(O)OCc2ccccc2)cc1</smiles>

(R, E)-diisopropyl (1-((4-methoxyphenyl)amino)-3-phenylallyl)phosphonate

4b: $75 \%$ yield (30 mg, n-pentane/EtOAc $=4: 1$ as the eluent). ${ }^{1} \mathbf{H} \mathbf{N M R}\left(\mathbf{4 0 0} \mathbf{M H z}, \mathbf{C D C l}_{3}\right) \delta 7.34(\mathrm{~d}, J$ $=7.5 \mathrm{~Hz}, 2 \mathrm{H}), 7.27(\mathrm{dd}, J=12.8,5.1 \mathrm{~Hz}, 2 \mathrm{H}), 7.21(\mathrm{~d}, J=7.2 \mathrm{~Hz}, 1 \mathrm{H}), 6.83-6.71(\mathrm{~m}, 2 \mathrm{H}), 6.71-$ $6.59(\mathrm{~m}, 3 \mathrm{H}), 6.34-6.10(\mathrm{~m}, 1 \mathrm{H}), 4.88-4.58(\mathrm{~m}, 2 \mathrm{H}), 4.33$ (ddd, $J=26.0,6.2,1.3 \mathrm{~Hz}, 1 \mathrm{H}), 3.71(\mathrm{~s}$, $3 \mathrm{H}), 1.33(\mathrm{dd}, J=6.2,1.8 \mathrm{~Hz}, 6 \mathrm{H}), 1.26(\mathrm{t}, J=5.7 \mathrm{~Hz}, 6 \mathrm{H}){ }^{\mathbf{1 3}} \mathbf{C} \mathbf{~ N M R}\left(\mathbf{1 0 1} \mathbf{M H z}, \mathbf{C D C l}_{\mathbf{3}}\right) \delta 152.53$, $140.72(\mathrm{~d}, J=13.6 \mathrm{~Hz}), 136.35$ (d, $J=3.3 \mathrm{~Hz}), 132.64$ (d, $J=12.6 \mathrm{~Hz}), 128.40,127.57,126.39$ (d, $J=$ $1.9 \mathrm{~Hz}), 124.02(\mathrm{~d}, J=4.3 \mathrm{~Hz}), 115.08,114.65,55.55,55.29$ (d, $J=154.5 \mathrm{~Hz}), 72.08$ (d, $J=7.3 \mathrm{~Hz})$, 71.60 (d, $J=7.5 \mathrm{~Hz}), 24.13$ (d, $J=3.2 \mathrm{~Hz}), 24.03$ (d, $J=3.4 \mathrm{~Hz}), 23.79$ (d, $J=5.4 \mathrm{~Hz}), 23.70$ (d, $J=$ $5.2 \mathrm{~Hz}){ }^{31} \mathbf{P}$ NMR $\left(\mathbf{1 6 2} \mathbf{~ M H z}, \mathbf{C D C l}_{\mathbf{3}}\right) \delta 20.78$.

Enantiomeric excess is $64 \%$ determined by HPLC analysis: Chiralcel AD-H (hexane/iPrOH $=50 / 50$, flow rate $=0.5 \mathrm{~mL} / \mathrm{min}, 240.4 \mathrm{~nm}), \mathrm{t}_{\mathrm{r}}($ major $)=12.516 \mathrm{~min}, \mathrm{t}_{\mathrm{r}}($ minor $)=18.113 \mathrm{~min} .[\alpha]_{\mathrm{D}}^{22.7}+45$ $\left(\mathrm{c}=1.0, \mathrm{CHCl}_{3}\right)\left(64 \%\right.$ ee). $\mathrm{lit}^{3}$ (R-form) $[\alpha]_{\mathrm{D}}{ }^{26}+72.3\left(\mathrm{c}=0.75, \mathrm{CHCl}_{3}\right)$. HRMS (ESI) for $\mathrm{C}_{22} \mathrm{H}_{31} \mathrm{NO}_{4} \mathrm{P}$ $\left([\mathrm{M}+\mathrm{H}]^{+}\right)$: calcd 404.1985, found 404.1992.
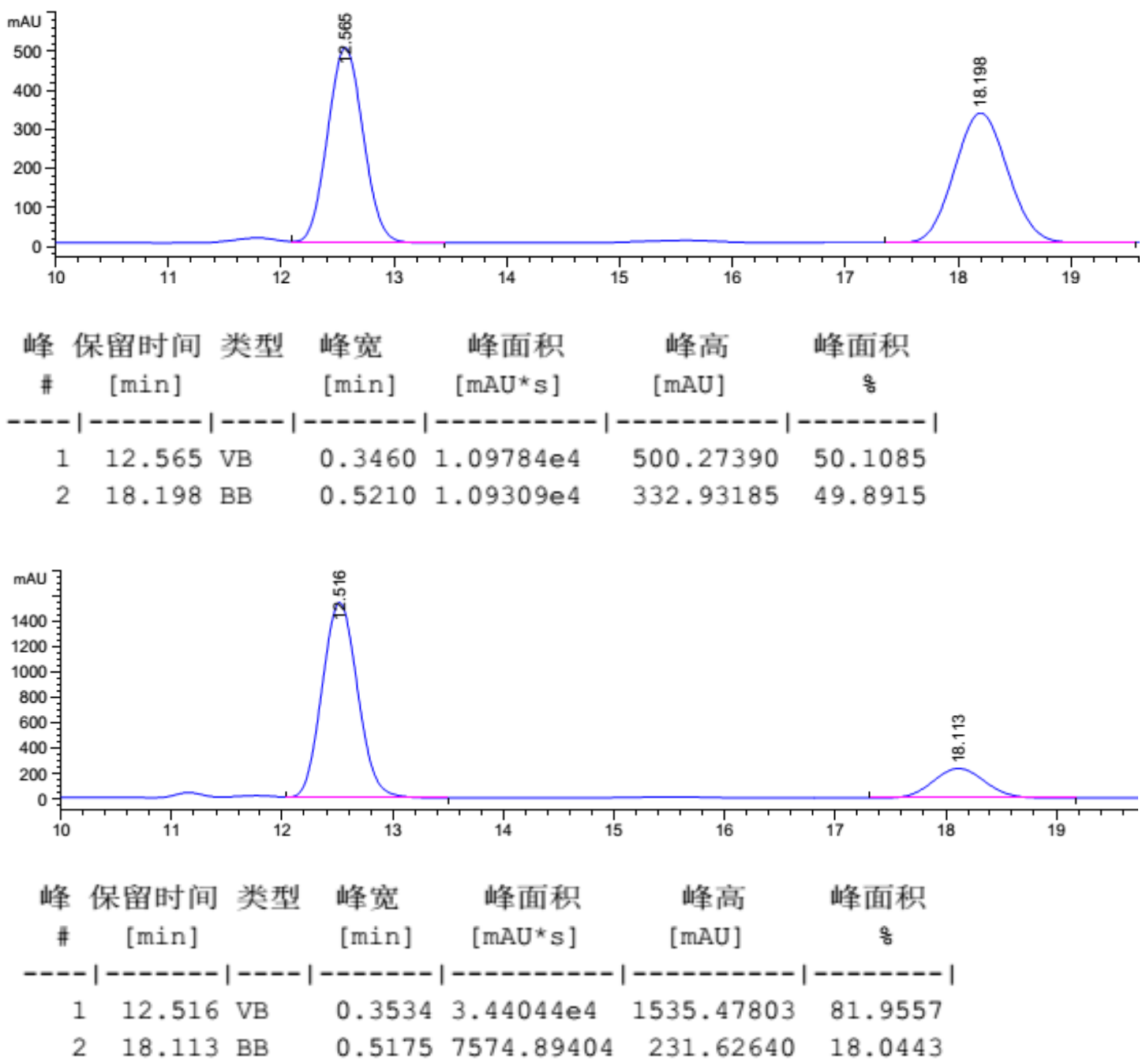
<smiles>O=C(P[PH](=O)O)C(/C=C/c1ccccc1)Nc1ccccc1</smiles>

( $R, E)$-diisopropyl (3-phenyl-1-(phenylamino)allyl)phosphonate

4c: $85 \%$ yield (32 mg, n-pentane/EtOAc $=4: 1$ as the eluent). ${ }^{\mathbf{1}} \mathbf{H}$ NMR (400 $\left.\mathbf{M H z}, \mathbf{C D C l}_{3}\right) \delta 7.35(\mathrm{~d}, J$ $=7.5 \mathrm{~Hz}, 2 \mathrm{H}), 7.29(\mathrm{t}, J=7.4 \mathrm{~Hz}, 2 \mathrm{H}), 7.26-7.20(\mathrm{~m}, 1 \mathrm{H}), 7.20-7.13(\mathrm{~m}, 2 \mathrm{H}), 6.71(\mathrm{dt}, J=13.2,6.3$ $\mathrm{Hz}, 4 \mathrm{H}), 6.42-6.08(\mathrm{~m}, 1 \mathrm{H}), 4.76(\mathrm{~m}, 2 \mathrm{H}), 4.50-4.21(\mathrm{~m}, 1 \mathrm{H} 2), 1.35$ (dd, $J=6.2,1.6 \mathrm{~Hz}, 6 \mathrm{H}), 1.27$ $(\mathrm{dd}, J=8.3,6.2 \mathrm{~Hz}, 6 \mathrm{H}) .{ }^{\mathbf{1 3}} \mathbf{C}$ NMR (101 MHz, $\left.\mathbf{C D C l}_{3}\right) \delta 146.69(\mathrm{~d}, J=12.6 \mathrm{~Hz}), 136.38(\mathrm{~d}, J=3.3$ $\mathrm{Hz}), 132.55$ (d, $J=12.4 \mathrm{~Hz}), 129.16,128.45,127.63,126.46$ (d, $J=1.9 \mathrm{~Hz}), 123.86$ (d, $J=4.5 \mathrm{~Hz})$, 118.29, 113.66, 71.98 (d, $J=7.2 \mathrm{~Hz}), 71.60$ (d, $J=7.3 \mathrm{~Hz}), 54.41(\mathrm{~d}, J=154.0 \mathrm{~Hz}), 24.19(\mathrm{~d}, J=3.3$ Hz), 24.09 (d, $J=3.5 \mathrm{~Hz}), 23.85$ (d, $J=5.3 \mathrm{~Hz}), 23.77$ (d, $J=5.2 \mathrm{~Hz}) .{ }^{31} \mathbf{P}$ NMR (162 MHz, $\left.\mathbf{C D C l}_{\mathbf{3}}\right) \delta$ 20.04. Enantiomeric excess is $71 \%$ determined by HPLC analysis: Chiralcel AD-H (hexane/iPrOH = $50 / 50$, flow rate $=0.5 \mathrm{~mL} / \mathrm{min}, 240.4 \mathrm{~nm}), \mathrm{t}_{\mathrm{r}}($ major $)=10.348 \mathrm{~min}, \mathrm{t}_{\mathrm{r}}($ minor $)=14.749 \mathrm{~min} .[\alpha]_{\mathrm{D}}^{22.7}$ $+32\left(\mathrm{c}=1.0, \mathrm{CHCl}_{3}\right)\left(71 \%\right.$ ee). HRMS (ESI) for $\mathrm{C}_{21} \mathrm{H}_{28} \mathrm{NO}_{3} \mathrm{PNa}\left([\mathrm{M}+\mathrm{Na}]^{+}\right)$: calcd 396.1699, found 396.1705.
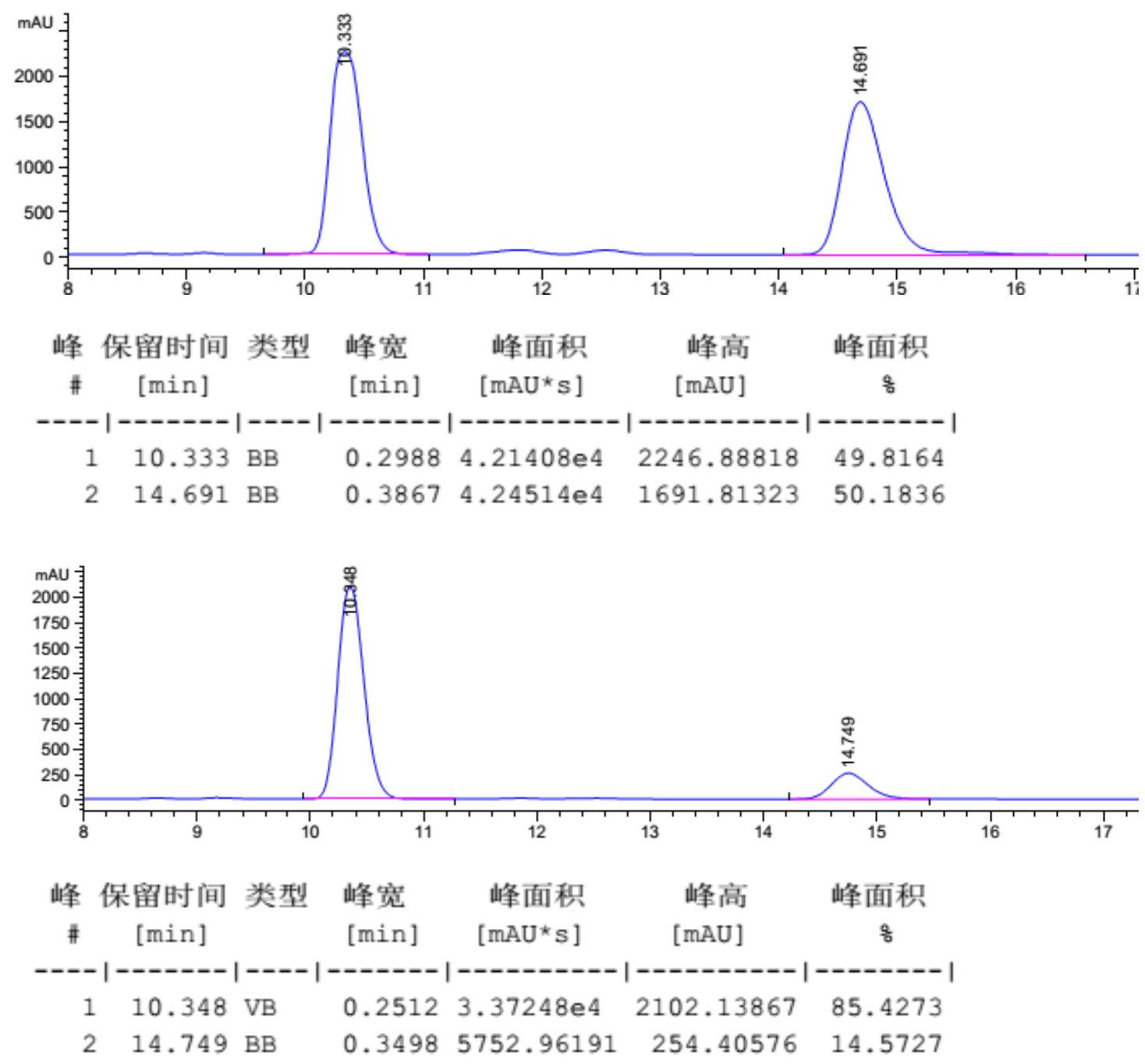
<smiles>Cc1ccccc1/C=C/[C@H](Nc1ccc(Br)cc1)P(=O)(O)O</smiles>

$(R, E)$-diisopropyl (1-((4-bromophenyl)amino)-3-(o-tolyl)allyl)phosphonate

4f: $73 \%$ yield (34mg, n-pentane/EtOAc $=4: 1$ as the eluent). ${ }^{1} \mathbf{H} \mathbf{~ N M R ~}\left(\mathbf{4 0 0} \mathbf{~ M H z}, \mathbf{C D C l}_{\mathbf{3}}\right) \delta 7.39(\mathrm{dd}, J$ $=5.1,3.9 \mathrm{~Hz}, 1 \mathrm{H}), 7.29-7.19(\mathrm{~m}, 2 \mathrm{H}), 7.18-7.09(\mathrm{~m}, 3 \mathrm{H}), 6.89-6.82(\mathrm{~m}, 1 \mathrm{H}), 6.58(\mathrm{~d}, J=8.9 \mathrm{~Hz}$, $2 \mathrm{H}), 6.13-6.02(\mathrm{~m}, 1 \mathrm{H}), 4.88-4.60(\mathrm{~m}, 2 \mathrm{H}), 4.51-4.14(\mathrm{~m}, 2 \mathrm{H}), 2.23(\mathrm{~s}, 3 \mathrm{H}), 1.35(\mathrm{dd}, J=6.2,1.8$ $\mathrm{Hz}, 6 \mathrm{H}), 1.28(\mathrm{dd}, J=10.3,6.2 \mathrm{~Hz}, 6 \mathrm{H}) .{ }^{13} \mathbf{C}$ NMR $\left(\mathbf{1 0 1} \mathbf{~ M H z}, \mathbf{C D C l}_{3}\right) \delta 145.70(\mathrm{~d}, J=13.2 \mathrm{~Hz})$, 135.52, 131.93, 130.99 (d, $J=12.3 \mathrm{~Hz}), 130.28,127.77,126.13,125.70$ (d, $J=2.2 \mathrm{~Hz}), 124.44$ (d, $J$ $=4.8 \mathrm{~Hz}), 115.48,110.10,72.06(\mathrm{~d}, J=7.2 \mathrm{~Hz}), 71.68(\mathrm{~d}, J=7.3 \mathrm{~Hz}), 54.65(\mathrm{~d}, J=154.4 \mathrm{~Hz}), 24.22$ (d, $J=3.4 \mathrm{~Hz}), 24.13(\mathrm{~d}, J=3.5 \mathrm{~Hz}), 23.95(\mathrm{~d}, J=5.2 \mathrm{~Hz}), 23.86(\mathrm{~d}, J=5.1 \mathrm{~Hz}), 19.70 .{ }^{31} \mathbf{P} \mathbf{~ N M R}$ $\left(162 \mathbf{~ M H z}, \mathbf{C D C l}_{3}\right) \delta 20.05$. Enantiomeric excess is $84 \%$ determined by HPLC analysis: Chiralcel $\mathrm{AD}-\mathrm{H}$ (hexane $/ \mathrm{PPOH}=50 / 50$, flow rate $=0.5 \mathrm{~mL} / \mathrm{min}, \quad 246.9 \mathrm{~nm}), \mathrm{t}_{\mathrm{r}}($ major $)=11.366 \mathrm{~min}, \mathrm{t}_{\mathrm{r}}($ minor $)$ $=13.375$ min. $[\alpha]_{\mathrm{D}}{ }^{20.4}+65\left(\mathrm{c}=1.0, \mathrm{CHCl}_{3}\right)(84 \%$ ee $)$. HRMS (ESI) for $\mathrm{C}_{22} \mathrm{H}_{29} \mathrm{BrNO}_{3} \mathrm{PNa}\left([\mathrm{M}+\mathrm{Na}]^{+}\right)$: calcd 488.0961, found 488.0971.
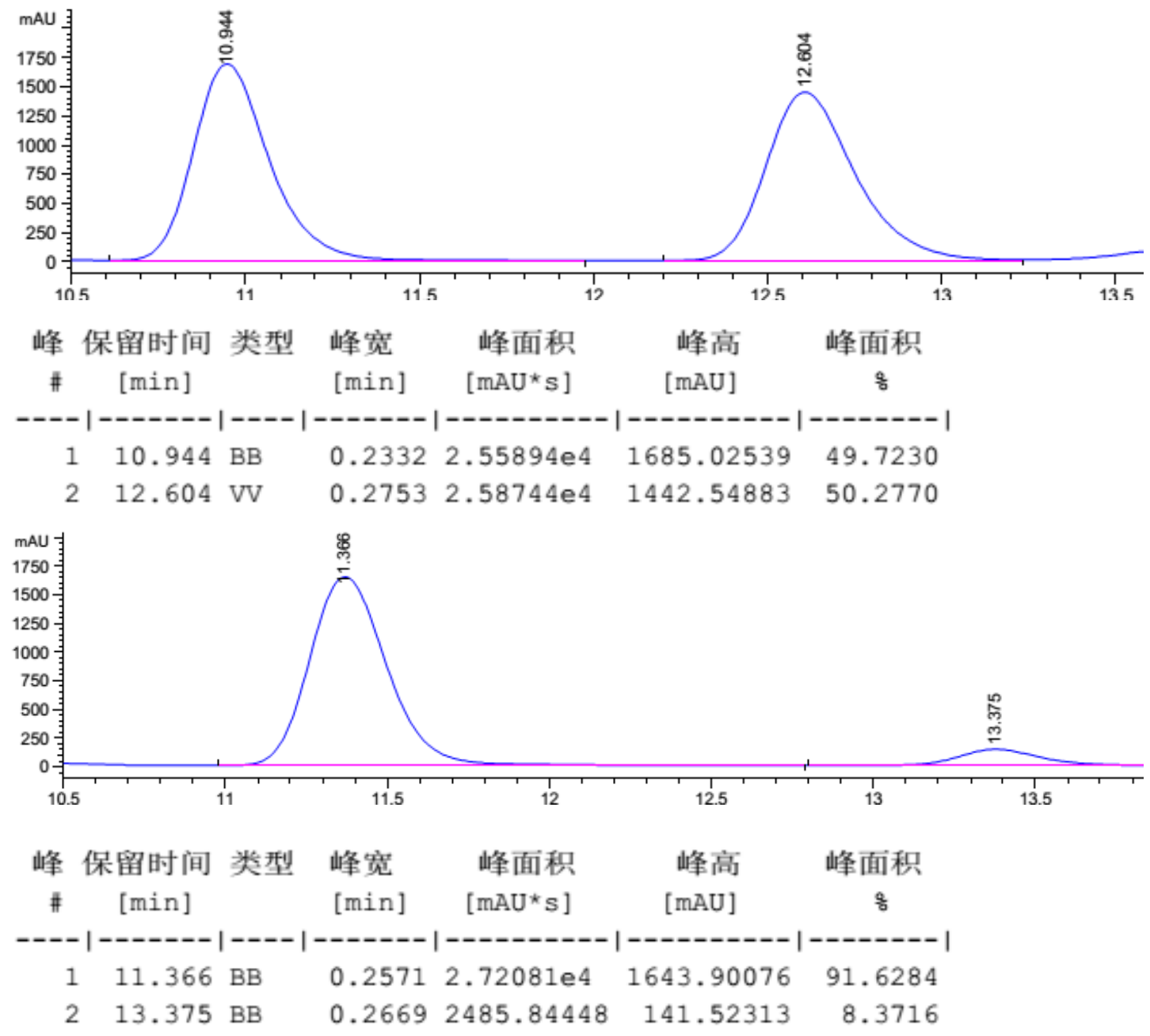
<smiles>O=[PH+]C(/C=C/c1ccccc1F)[PH](=O)OCc1ccc(Br)cc1</smiles>

$(R, E)$-diisopropyl (1-((4-bromophenyl)amino)-3-(2-fluorophenyl)allyl)phosphonate 4g: 77\% yield (36 mg, n-pentane/EtOAc $=4: 1$ as the eluent). ${ }^{1} \mathbf{H} \mathbf{N M R}\left(\mathbf{4 0 0} \mathbf{~ M H z}, \mathbf{C D C l}_{\mathbf{3}}\right) \delta 7.40$ (t, $J$ $=7.3 \mathrm{~Hz}, 1 \mathrm{H}), 7.30-7.23(\mathrm{~m}, 2 \mathrm{H}), 7.19(\mathrm{dd}, J=10.3,4.2 \mathrm{~Hz}, 1 \mathrm{H}), 7.11-6.97(\mathrm{~m}, 2 \mathrm{H}), 6.80(\mathrm{dd}, J=$ 16.0, $4.7 \mathrm{~Hz}, 1 \mathrm{H}), 6.57(\mathrm{~d}, J=8.8 \mathrm{~Hz}, 2 \mathrm{H}), 6.38-6.28(\mathrm{~m}, 1 \mathrm{H}), 4.89-4.61(\mathrm{~m}, 2 \mathrm{H}), 4.46-4.18(\mathrm{~m}$, $2 \mathrm{H}), 1.35(\mathrm{dd}, J=6.1,2.3 \mathrm{~Hz}, 6 \mathrm{H}), 1.27(\mathrm{dd}, J=7.9,6.3 \mathrm{~Hz}, 6 \mathrm{H}) .{ }^{13} \mathbf{C} \mathbf{~ N M R}\left(\mathbf{1 0 1} \mathbf{~ M H z}, \mathbf{C D C l}_{\mathbf{3}}\right) \delta$ $145.63(\mathrm{~d}, J=12.7 \mathrm{~Hz}), 132.00,129.12(\mathrm{~d}, J=7.9 \mathrm{~Hz}), 127.71,125.43$ (d, $J=12.5 \mathrm{~Hz}), 124.08$ (d, $J=$ $4.0 \mathrm{~Hz}), 115.75$ (d, $J=22.2 \mathrm{~Hz}), 115.34,110.14,72.17$ (d, $J=7.2 \mathrm{~Hz}), 71.87(\mathrm{~d}, J=7.3 \mathrm{~Hz}), 54.68$ (d, $J=153.9 \mathrm{~Hz}), 24.20(\mathrm{~d}, J=3.3 \mathrm{~Hz}), 24.13(\mathrm{~d}, J=3.5 \mathrm{~Hz}), 23.83(\mathrm{~d}, J=5.3 \mathrm{~Hz}), 23.79(\mathrm{~d}, J=5.3 \mathrm{~Hz})$. ${ }^{31} \mathbf{P}$ NMR (162 MHz, $\left.\mathbf{C D C l}_{3}\right) \delta 19.68 .{ }^{19} \mathbf{F}$ NMR (376 $\left.\mathbf{~ M H z}, \mathbf{C D C l}_{3}\right) \delta-117.57$. Enantiomeric excess is $85 \%$ determined by HPLC analysis: Chiralcel AD-H (hexane $/ \mathrm{iPrOH}=50 / 50$, flow rate $=0.5 \mathrm{~mL} / \mathrm{min}$, $240.4 \mathrm{~nm}), \mathrm{t}_{\mathrm{r}}$ (major) $=12.275 \mathrm{~min}, \mathrm{t}_{\mathrm{r}}($ minor $)=16.837 \mathrm{~min} .[\alpha]_{\mathrm{D}}^{20.2}+59\left(\mathrm{c}=1.0, \mathrm{CHCl}_{3}\right)(85 \% \mathrm{ee})$. HRMS (ESI) for $\mathrm{C}_{21} \mathrm{H}_{26} \mathrm{BrFNO}_{3} \mathrm{PNa}\left([\mathrm{M}+\mathrm{Na}]^{+}\right)$: calcd 492.0710, found 492.0718 .
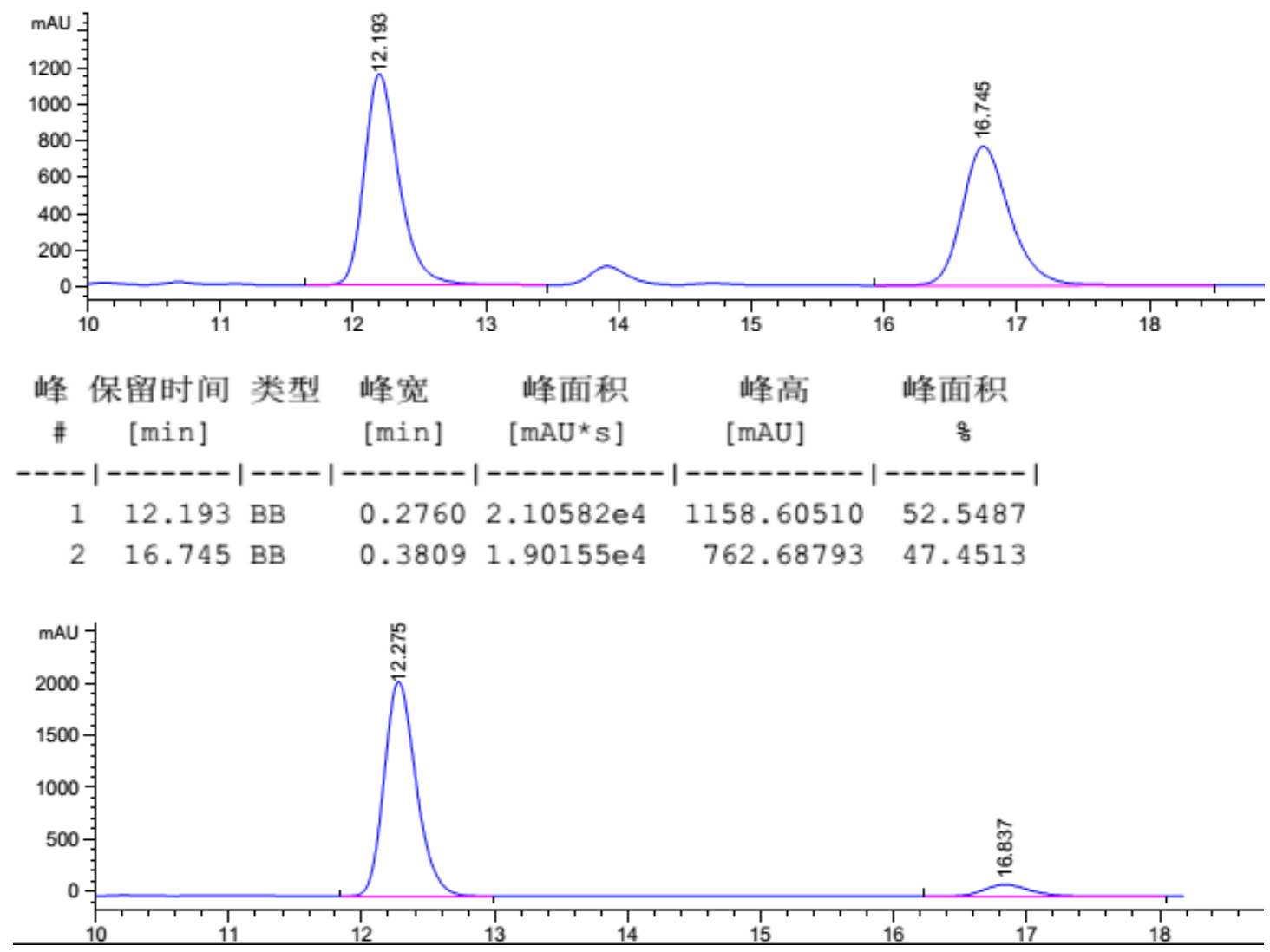


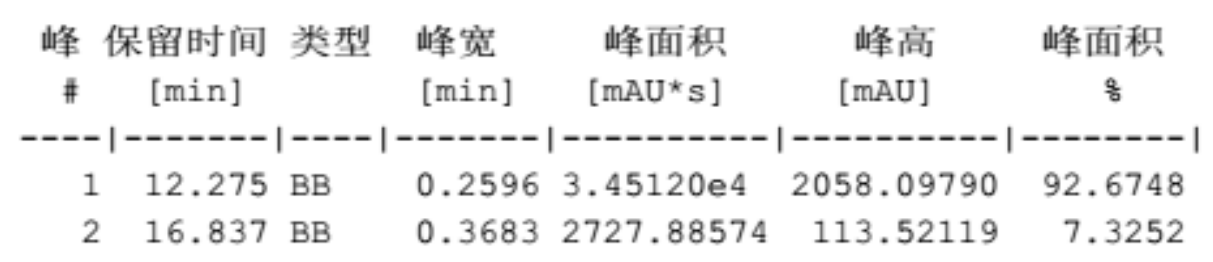<smiles>O=[PH+]P(=O)(O)[C@H](/C=C/c1ccccc1Cl)Nc1ccc(Br)cc1</smiles>

$(R, E)$-diisopropyl (1-((4-bromophenyl)amino)-3-(2-chlorophenyl)allyl)phosphonate

4h: $64 \%$ yield (31 mg, n-pentane/EtOAc $=4: 1$ as the eluent). ${ }^{1} \mathbf{H} \mathbf{~ N M R}\left(\mathbf{4 0 0} \mathbf{~ M H z}, \mathbf{C D C l}_{\mathbf{3}}\right) \delta 7.48(\mathrm{dd}$, $J=7.4,1.8 \mathrm{~Hz}, 1 \mathrm{H}), 7.33(\mathrm{dd}, J=7.5,1.7 \mathrm{~Hz}, 1 \mathrm{H}), 7.30-7.23(\mathrm{~m}, 2 \mathrm{H}), 7.19-7.17(\mathrm{~m}, 2 \mathrm{H}), 7.06(\mathrm{dd}, J$ $=15.9,4.6 \mathrm{~Hz}, 1 \mathrm{H}), 6.59(\mathrm{~d}, J=8.8 \mathrm{~Hz}, 2 \mathrm{H}), 6.21(\mathrm{dt}, J=15.9,5.3 \mathrm{~Hz}, 1 \mathrm{H}), 4.85-4.65(\mathrm{~m}, 2 \mathrm{H}), 4.38$ $(\mathrm{dt}, J=18.9,8.6 \mathrm{~Hz}, 2 \mathrm{H}), 1.35(\mathrm{~d}, J=6.2 \mathrm{~Hz}, 6 \mathrm{H}), 1.28(\mathrm{dd}, J=6.0,4.5 \mathrm{~Hz}, 6 \mathrm{H}) .{ }^{13} \mathbf{C}$ NMR $(\mathbf{1 0 1} \mathbf{M H z}$, $\left.\mathbf{C D C l}_{3}\right) \delta 145.62(\mathrm{~d}, J=13.0 \mathrm{~Hz}), 134.41,133.07,131.94,129.67,129.39(\mathrm{~d}, J=12.4 \mathrm{~Hz}), 128.85$, 126.94, 126.84, 126.48 (d, $J=4.6 \mathrm{~Hz}), 115.41,110.19,72.23(\mathrm{~d}, J=7.3 \mathrm{~Hz}), 71.84(\mathrm{~d}, J=7.3 \mathrm{~Hz})$, $54.70(\mathrm{~d}, J=154.0 \mathrm{~Hz}), 24.22(\mathrm{~d}, J=3.3 \mathrm{~Hz}), 24.12(\mathrm{~d}, J=3.6 \mathrm{~Hz}), 23.91(\mathrm{~d}, J=5.8 \mathrm{~Hz}), 23.86(\mathrm{~d}, J=$ $5.5 \mathrm{~Hz}) .{ }^{31} \mathbf{P}$ NMR (162 $\left.\mathbf{~ M H z}, \mathbf{C D C l}_{3}\right) \delta 19.53$. Enantiomeric excess is $90 \%$ determined by HPLC analysis: Chiralcel AD-H (hexane $/ \mathrm{PrOH}=50 / 50$, flow rate $=0.5 \mathrm{~mL} / \mathrm{min}, 230.4 \mathrm{~nm}), \mathrm{t}_{\mathrm{r}}($ major $)=$ $12.591 \mathrm{~min}, \mathrm{t}_{\mathrm{r}}$ (minor) $=14.204 \mathrm{~min} .[\alpha]_{\mathrm{D}}^{21.2}+42\left(\mathrm{c}=1.0, \mathrm{CHCl}_{3}\right)(90 \%$ ee $)$. HRMS (ESI) for $\mathrm{C}_{21} \mathrm{H}_{26} \mathrm{BrClNO}_{3} \mathrm{PNa}\left([\mathrm{M}+\mathrm{Na}]^{+}\right)$: calcd 508.0414, found 508.0410
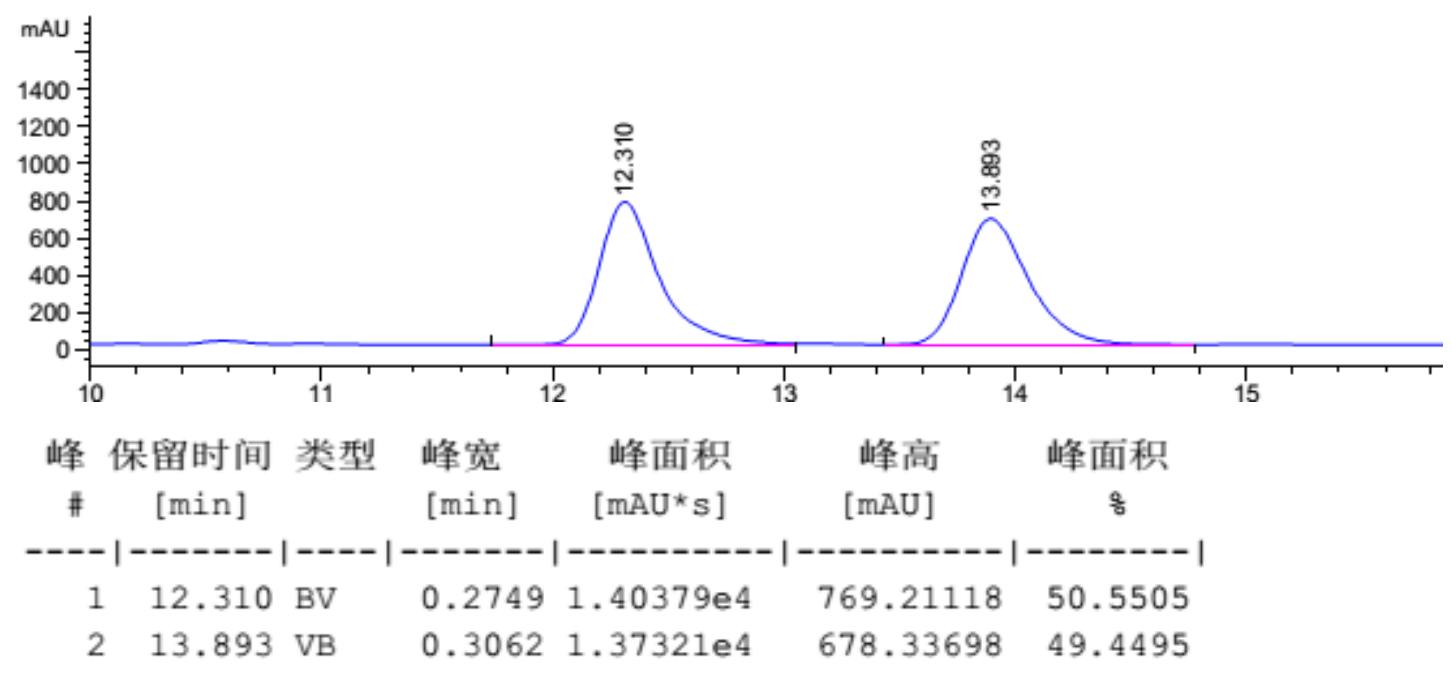

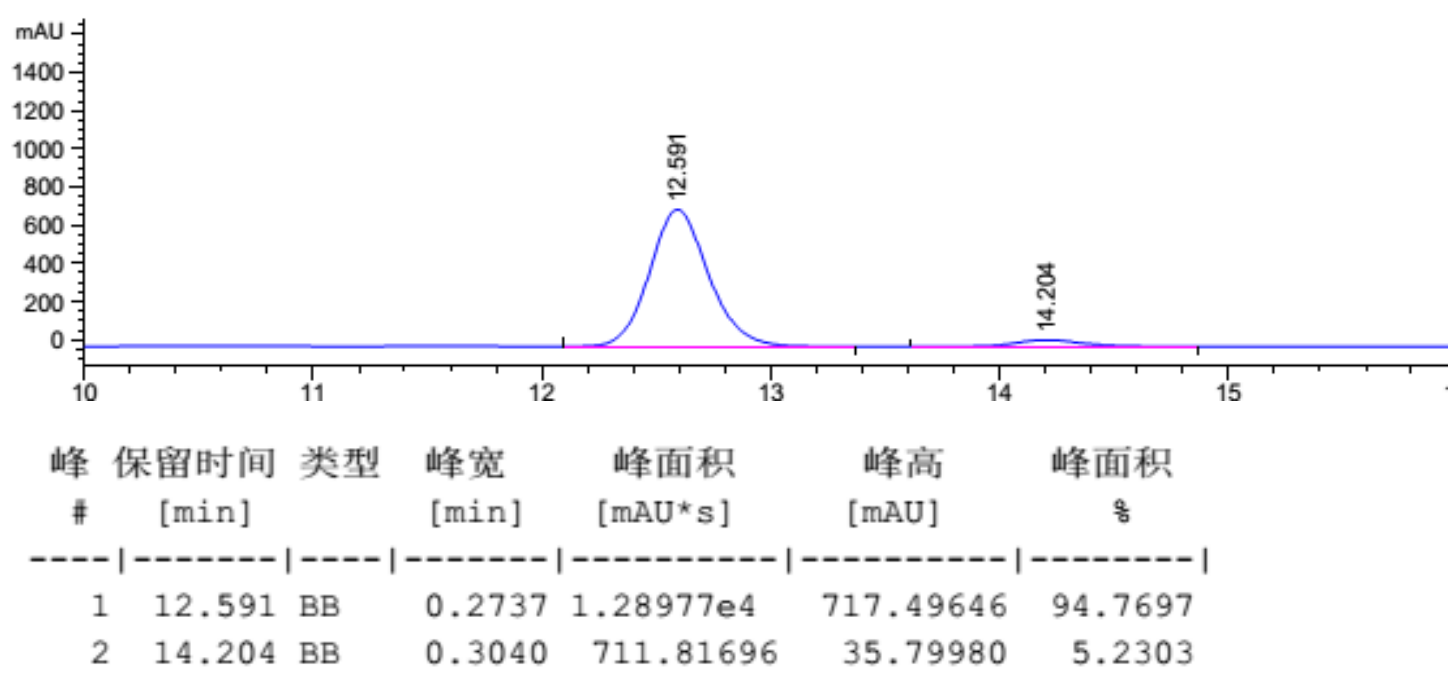<smiles>O=[PH+]([O-])[C@H](/C=C/c1ccccc1C(F)(F)F)Nc1ccc(Br)cc1</smiles>

$(R, E)$-diisopropyl (1-((4-bromophenyl)amino)-3-(2-(trifluoromethyl)phenyl)allyl)phosphonate 4i: $92 \%$ yield (48mg, n-pentane/EtOAc $=4: 1$ as the eluent). ${ }^{1} \mathbf{H} \mathbf{~ N M R ~}\left(\mathbf{4 0 0} \mathbf{~ M H z}, \mathbf{C D C l}_{\mathbf{3}}\right) \delta 7.59(\mathrm{dd}, J$ $=13.8,7.9 \mathrm{~Hz}, 2 \mathrm{H}), 7.48(\mathrm{t}, J=7.6 \mathrm{~Hz}, 1 \mathrm{H}), 7.34(\mathrm{t}, J=7.6 \mathrm{~Hz}, 1 \mathrm{H}), 7.25(\mathrm{~d}, J=8.9 \mathrm{~Hz}, 2 \mathrm{H}), 7.04(\mathrm{~d}$, $J=15.4 \mathrm{~Hz}, 1 \mathrm{H}), 6.58(\mathrm{~d}, J=8.8 \mathrm{~Hz}, 2 \mathrm{H}), 6.20(\mathrm{dt}, J=15.7,5.3 \mathrm{~Hz}, 1 \mathrm{H}), 4.89-4.62(\mathrm{~m}, 2 \mathrm{H}), 4.50-$ $4.16(\mathrm{~m}, 2 \mathrm{H}), 1.35(\mathrm{dd}, J=6.1,1.7 \mathrm{~Hz}, 6 \mathrm{H}), 1.28(\mathrm{dd}, J=6.0,3.5 \mathrm{~Hz}, 6 \mathrm{H}) .{ }^{13} \mathbf{C}$ NMR (101 MHz, $\left.\mathbf{C D C l}_{3}\right) \delta 145.55(\mathrm{~d}, J=13.3 \mathrm{~Hz}), 135.27,131.89,131.84,129.19(\mathrm{~d}, J=12.4 \mathrm{~Hz}), 128.32(\mathrm{~d}, J=4.5$ $\mathrm{Hz}), 127.52,127.44(\mathrm{~d}, J=2.1 \mathrm{~Hz}), 125.81(\mathrm{t}, J=46.4 \mathrm{~Hz}), 124.10(\mathrm{~d}, J=273.9 \mathrm{~Hz}), 122.74,110.26$, 72.26 (d, $J=7.1 \mathrm{~Hz}), 71.79$ (d, $J=7.3 \mathrm{~Hz}), 54.75(\mathrm{~d}, J=154.0 \mathrm{~Hz}), 24.17$ (d, $J=3.4 \mathrm{~Hz}), 24.04$ (d, $J=$ $3.7 \mathrm{~Hz}), 23.88(\mathrm{~d}, J=5.1 \mathrm{~Hz}), 23.79(\mathrm{~d}, J=5.1 \mathrm{~Hz}) .{ }^{31} \mathbf{P} \mathbf{~ N M R}\left(\mathbf{1 6 2} \mathbf{~ M H z}, \mathbf{C D C l}_{3}\right) \delta 19.41 .{ }^{\mathbf{1 9}} \mathbf{F} \mathbf{~ N M R}$ $\left(376 \mathbf{~ M H z}, \mathbf{C D C l}_{3}\right) \delta$-59.41. Enantiomeric excess is $90 \%$ determined by HPLC analysis: Chiralcel $\mathrm{AD}-\mathrm{H}$ (hexane $/ \mathrm{PrOH}=75 / 25$, flow rate $=0.5 \mathrm{~mL} / \mathrm{min}, 240.4 \mathrm{~nm}), \mathrm{t}_{\mathrm{r}}$ (major) $=12.480 \mathrm{~min}, \mathrm{t}_{\mathrm{r}}($ minor $)$ $=14.389 \mathrm{~min} .[\alpha]_{\mathrm{D}}{ }^{20.8}+44\left(\mathrm{c}=1.0, \mathrm{CHCl}_{3}\right)\left(90 \%\right.$ ee). HRMS (ESI) for $\mathrm{C}_{22} \mathrm{H}_{26} \mathrm{BrF}_{3} \mathrm{NO}_{3} \mathrm{PNa}$ $\left([\mathrm{M}+\mathrm{Na}]^{+}\right)$: calcd 542.0678, found 542.0688.

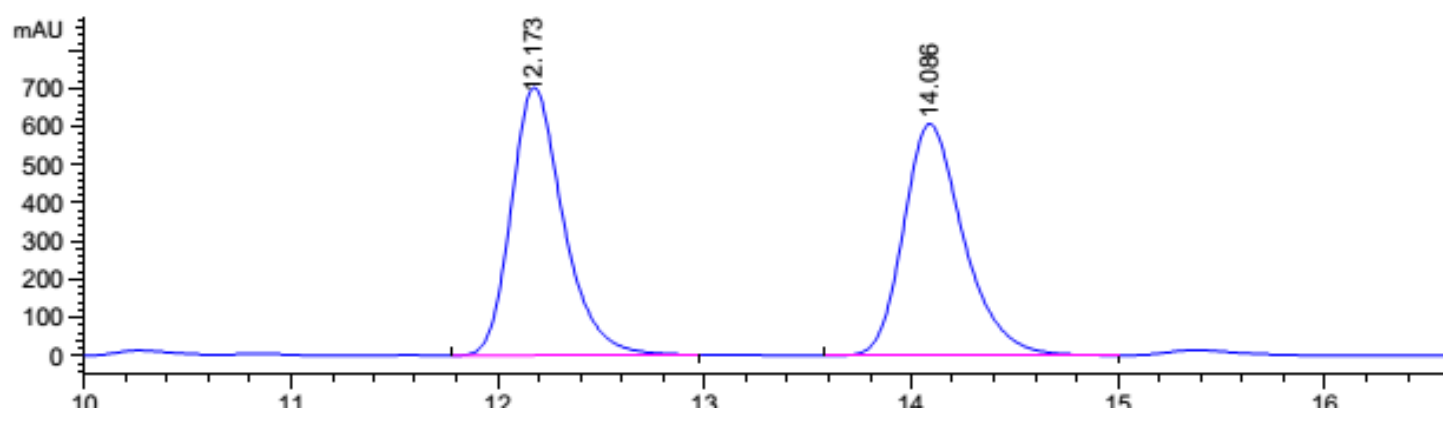



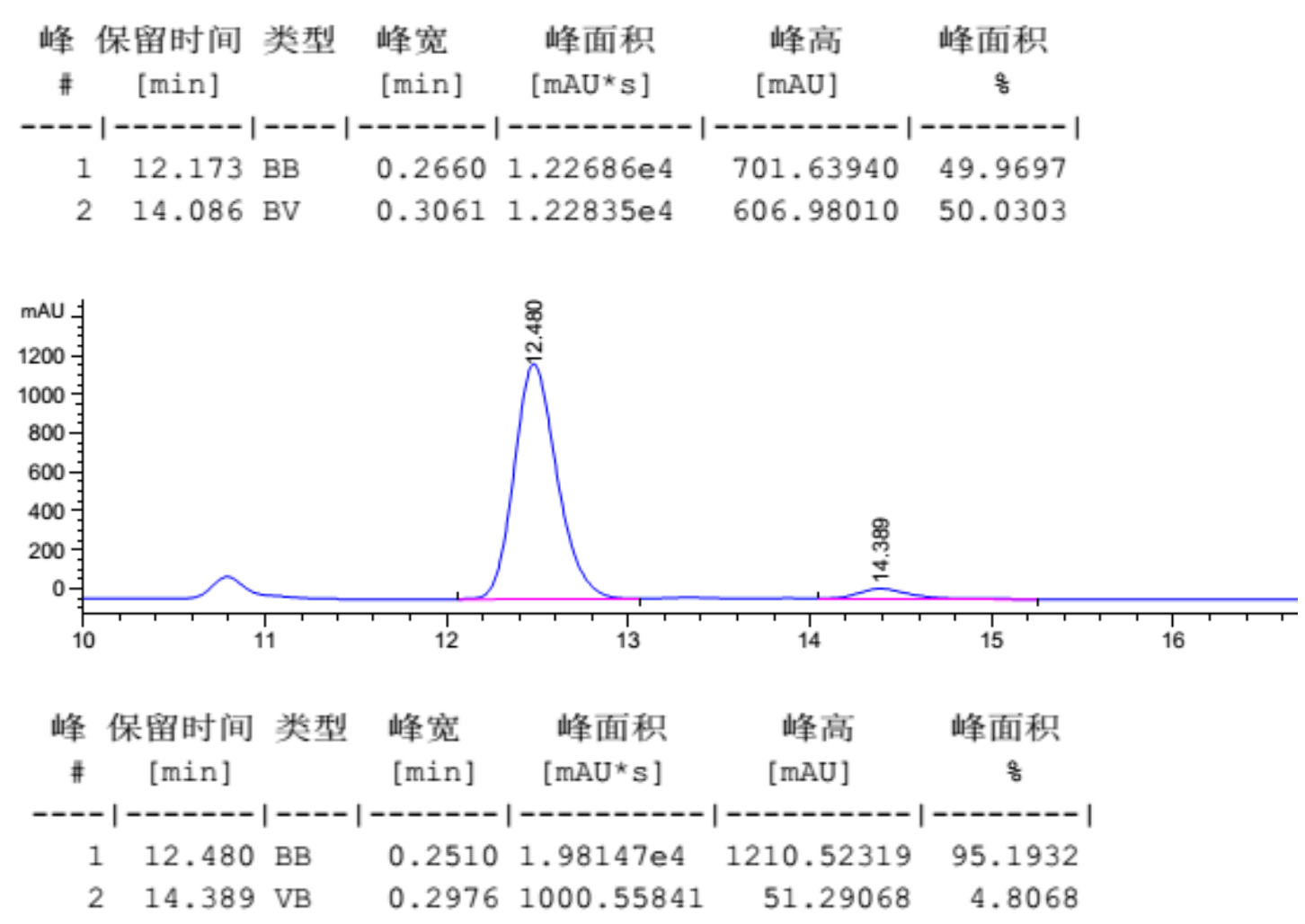<smiles>O=[N+]([O-])c1ccccc1/C=C/[C@H](Nc1ccc(Br)cc1)P(=O)(O)O</smiles>

(R, E)-diisopropyl (1-((4-bromophenyl)amino)-3-(2-nitrophenyl)allyl)phosphonate

4j: $88 \%$ yield (44 mg, n-pentane/EtOAc $=4: 1$ as the eluent). ${ }^{1} \mathbf{H} \mathbf{~ N M R ~}\left(\mathbf{4 0 0} \mathbf{~ M H z}, \mathbf{C D C l}_{\mathbf{3}}\right) \delta 7.93(\mathrm{~d}, J$ $=8.1 \mathrm{~Hz}, 1 \mathrm{H}), 7.63-7.49(\mathrm{~m}, 2 \mathrm{H}), 7.47-7.31(\mathrm{~m}, 1 \mathrm{H}), 7.33-7.22(\mathrm{~m}, 2 \mathrm{H}), 7.22-7.03(\mathrm{~m}, 1 \mathrm{H}), 6.68$ $-6.51(\mathrm{~m}, 2 \mathrm{H}), 6.35-6.06(\mathrm{~m}, 1 \mathrm{H}), 4.92-4.62(\mathrm{~m}, 2 \mathrm{H}), 4.51-4.15(\mathrm{~m}, 2 \mathrm{H}), 1.35(\mathrm{dd}, J=6.2,3.4 \mathrm{~Hz}$, 6H), 1.29 (d, $J=6.2 \mathrm{~Hz}, 6 \mathrm{H}) .{ }^{13} \mathbf{C}$ NMR (101 MHz, $\left.\mathbf{C D C l}_{\mathbf{3}}\right) \delta 147.53,145.48$ (d, $\left.J=12.9 \mathrm{~Hz}\right), 133.17$, $132.13(\mathrm{~d}, J=2.9 \mathrm{~Hz}), 131.99,129.41(\mathrm{~d}, J=4.2 \mathrm{~Hz}), 128.80(\mathrm{~d}, J=2.3 \mathrm{~Hz}), 128.64(\mathrm{~d}, J=12.5 \mathrm{~Hz})$, 128.38, 124.61, 115.39, 110.38, $72.44(\mathrm{~d}, J=7.1 \mathrm{~Hz}), 71.91(\mathrm{~d}, J=7.3 \mathrm{~Hz}), 54.70(\mathrm{~d}, J=153.5 \mathrm{~Hz})$, 24.17 (d, $J=3.3 \mathrm{~Hz}), 24.04$ (d, $J=3.8 \mathrm{~Hz}), 23.91$ (d, $J=5.1 \mathrm{~Hz}), 23.83$ (d, $J=5.1 \mathrm{~Hz}) .{ }^{31} \mathbf{P}$ NMR (162 $\left.\mathbf{M H z}, \mathbf{C D C l}_{3}\right) \delta$ 19.03. Enantiomeric excess is $86 \%$ determined by HPLC analysis: Chiralcel AD-H (hexane $/ \mathrm{PrOH}=50 / 50$, flow rate $=0.5 \mathrm{~mL} / \mathrm{min}, 240.4 \mathrm{~nm}), \mathrm{t}_{\mathrm{r}}($ major $)=15.726 \mathrm{~min}, \mathrm{t}_{\mathrm{r}}($ minor $)=$ 19.026 min. $[\alpha]_{\mathrm{D}}{ }^{19.9}+104\left(\mathrm{c}=1.0, \mathrm{CHCl}_{3}\right)\left(86 \%\right.$ ee). HRMS (ESI) for $\mathrm{C}_{21} \mathrm{H}_{26} \mathrm{BrN}_{2} \mathrm{O}_{5} \mathrm{PNa}\left([\mathrm{M}+\mathrm{Na}]^{+}\right)$: calcd 519.0655, found 519.0664 . 

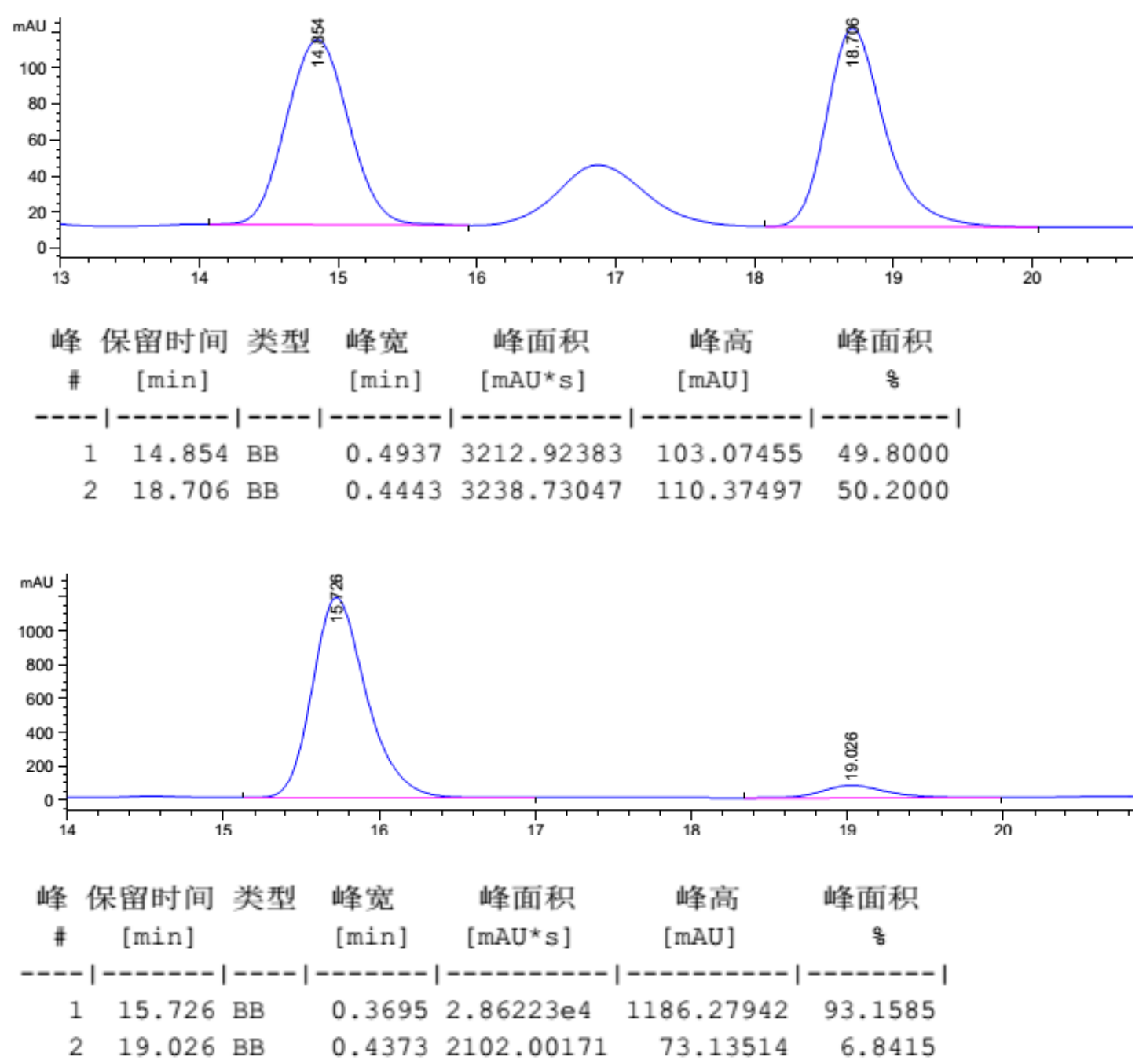<smiles>Cc1cccc(/C=C/[C@H](Nc2ccc(Br)cc2)P(=O)(O)OCc2ccccc2)c1</smiles>

( $R, E)$-diisopropyl (1-((4-bromophenyl)amino)-3-(m-tolyl)allyl)phosphonate

4k: $90 \%$ yield $\left(42 \mathrm{mg}, \mathrm{n}\right.$-pentane/EtOAc $=4: 1$ as the eluent). ${ }^{\mathbf{1}} \mathbf{H} \mathbf{~ N M R}\left(\mathbf{4 0 0} \mathbf{~ M H z}, \mathbf{C D C l}_{\mathbf{3}}\right) \delta 7.28-$ $7.21(\mathrm{~m}, 2 \mathrm{H}), 7.21-7.11(\mathrm{~m}, 3 \mathrm{H}), 7.05(\mathrm{~d}, J=7.0 \mathrm{~Hz}, 1 \mathrm{H}), 6.65-6.51(\mathrm{~m}, 3 \mathrm{H}), 6.28-6.16(\mathrm{~m}, 1 \mathrm{H})$, $4.87-4.61(\mathrm{~m}, 2 \mathrm{H}), 4.44-4.20(\mathrm{~m}, 2 \mathrm{H}), 2.32(\mathrm{~s}, 3 \mathrm{H}), 1.34(\mathrm{dd}, J=6.2,1.9 \mathrm{~Hz}, 6 \mathrm{H}), 1.26(\mathrm{dd}, J=7.7$, $6.3 \mathrm{~Hz}, 6 \mathrm{H}) .{ }^{13} \mathbf{C}$ NMR $\left(\mathbf{1 0 1} \mathbf{~ M H z}, \mathbf{C D C l}_{\mathbf{3}}\right) \delta 145.70(\mathrm{~d}, J=12.9 \mathrm{~Hz}), 138.11,136.05(\mathrm{~d}, J=3.2 \mathrm{~Hz})$, $132.87(\mathrm{~d}, J=12.3 \mathrm{~Hz}), 131.90,128.51(\mathrm{~d}, J=19.7 \mathrm{~Hz}), 127.25,123.59,122.90(\mathrm{~d}, J=4.7 \mathrm{~Hz}), 115.24$ 109.94, 72.07 (d, $J=7.2 \mathrm{~Hz}), 71.77(\mathrm{~d}, J=7.3 \mathrm{~Hz}), 54.38$ (d, $J=154.4 \mathrm{~Hz}), 24.20(\mathrm{~d}, J=3.3 \mathrm{~Hz})$, $24.13(\mathrm{~d}, J=3.4 \mathrm{~Hz}), 23.88$ (d, $J=5.6 \mathrm{~Hz}), 23.83(\mathrm{~d}, J=5.5 \mathrm{~Hz}), 21.32 .{ }^{31} \mathbf{P} \mathbf{~ N M R}\left(\mathbf{1 6 2} \mathbf{~ M H z}, \mathbf{C D C l}_{\mathbf{3}}\right)$ $\delta$ 20.05. Enantiomeric excess is $84 \%$ determined by HPLC analysis: Chiralcel AD-H (hexane/ $\mathrm{PrOH}=$ $50 / 50$, flow rate $=0.5 \mathrm{~mL} / \mathrm{min}, 230.4 \mathrm{~nm}), \mathrm{t}_{\mathrm{r}}($ major $)=9.967 \mathrm{~min}, \mathrm{t}_{\mathrm{r}}($ minor $)=163.680 \mathrm{~min} .[\alpha]_{\mathrm{D}}{ }^{20}+58$ 
$\left(\mathrm{c}=1.0, \mathrm{CHCl}_{3}\right)\left(84 \%\right.$ ee). HRMS (ESI) for $\mathrm{C}_{22} \mathrm{H}_{29} \mathrm{BrNO}_{3} \mathrm{PNa}\left([\mathrm{M}+\mathrm{Na}]^{+}\right)$: calcd 488.0961, found 488.0973 .
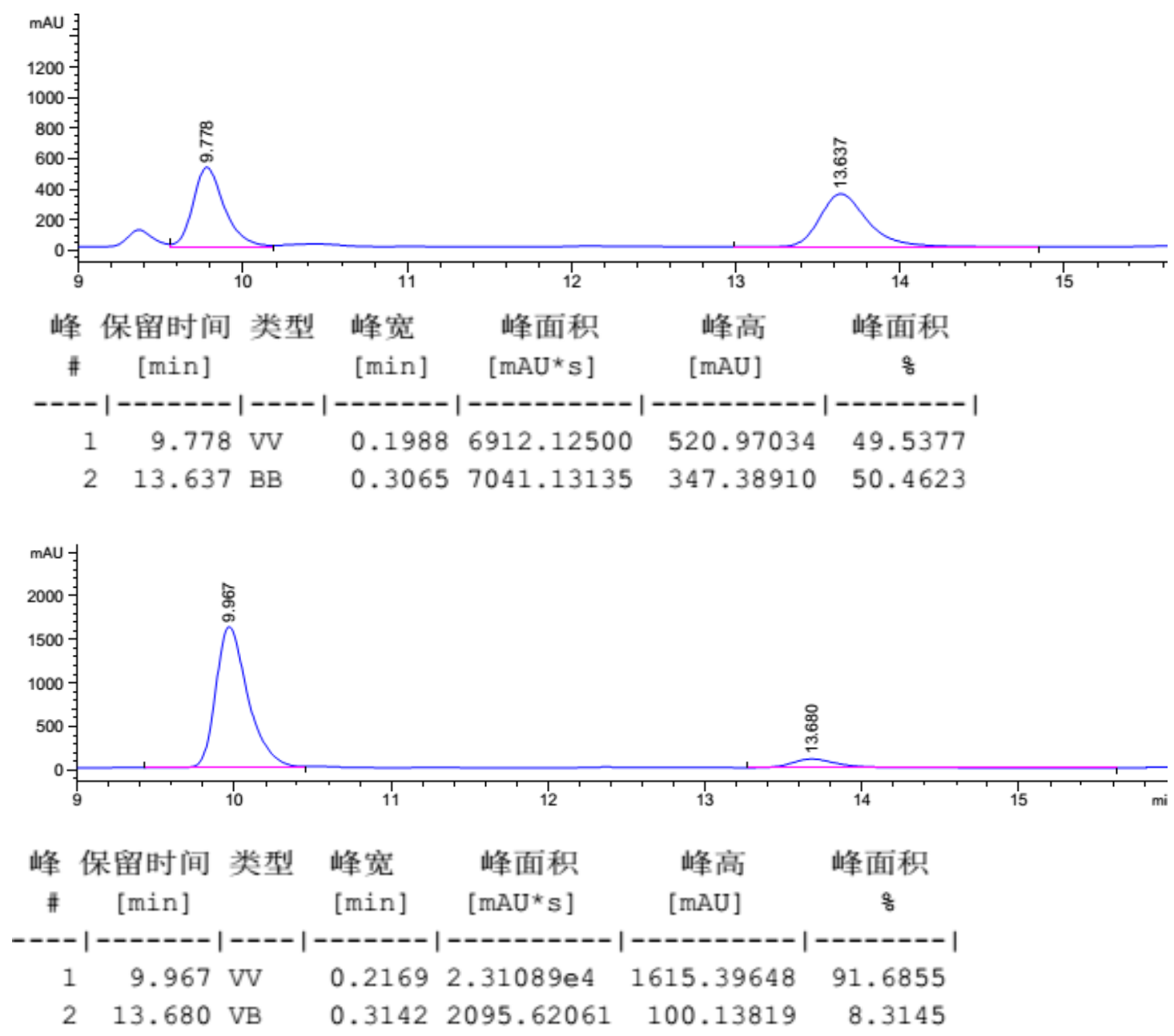<smiles>O=C(O[PH](=O)O)[C@H](/C=C/c1cccc(Cl)c1)Nc1ccc(Br)cc1</smiles>

( $R, E)$-diisopropyl (1-((4-bromophenyl)amino)-3-(3-chlorophenyl)allyl)phosphonate

41: $95 \%$ yield (46 mg, n-pentane/EtOAc $=4: 1$ as the eluent). ${ }^{1} \mathbf{H} \mathbf{N M R}\left(\mathbf{4 0 0} \mathbf{~ M H z}, \mathbf{C D C l}_{\mathbf{3}}\right) \delta 7.31$ (s, 1H), $7.29-7.17$ (m, 5H), $6.64-6.49(\mathrm{~m}, 3 \mathrm{H}), 6.27(\mathrm{dd}, J=16.0,5.7 \mathrm{~Hz}, 1 \mathrm{H}), 4.75(\mathrm{~m}, 2 \mathrm{H}), 4.33$ (d, $J$ $=26.9 \mathrm{~Hz}, 2 \mathrm{H}), 1.34(\mathrm{dd}, J=6.1,1.5 \mathrm{~Hz}, 5 \mathrm{H}), 1.26(\mathrm{dd}, J=10.6,6.2 \mathrm{~Hz}, 5 \mathrm{H}) .{ }^{13} \mathbf{C} \mathbf{N M R}(\mathbf{1 0 1} \mathbf{~ M H z}$, $\left.\mathbf{C D C l}_{3}\right) \delta 145.60(\mathrm{~d}, J=12.6 \mathrm{~Hz}), 138.05(\mathrm{~d}, J=3.3 \mathrm{~Hz}), 134.51,131.99,131.31(\mathrm{~d}, J=12.2 \mathrm{~Hz})$, 129.76, 127.74, 126.44 (d, $J=1.9 \mathrm{~Hz}), 125.06$ (d, $J=4.6 \mathrm{~Hz}), 124.64$ (d, $J=1.8 \mathrm{~Hz}), 115.21,110.16$, 72.23 (d, $J=7.2 \mathrm{~Hz}), 71.87$ (d, $J=7.4 \mathrm{~Hz}), 54.33$ (d, $J=153.9 \mathrm{~Hz}), 24.18$ (d, $J=3.4 \mathrm{~Hz}), 24.09$ (d, $J=$ $3.5 \mathrm{~Hz}), 23.90(\mathrm{~d}, J=5.2 \mathrm{~Hz}), 23.81(\mathrm{~d}, J=5.1 \mathrm{~Hz}) .{ }^{31} \mathbf{P}$ NMR $\left(\mathbf{1 6 2} \mathbf{~ M H z}, \mathbf{C D C l}_{\mathbf{3}}\right) \delta 19.53$. Enantiomeric excess is $83 \%$ determined by HPLC analysis: Chiralcel AD-H (hexane/iPrOH $=50 / 50$, flow rate $=0.5 \mathrm{~mL} / \mathrm{min}, 230.4 \mathrm{~nm}), \mathrm{t}_{\mathrm{r}}($ major $)=10.445 \mathrm{~min}, \mathrm{t}_{\mathrm{r}}($ minor $)=15.625 \mathrm{~min} .[\alpha]_{\mathrm{D}}^{22}+81(\mathrm{c}=1.0$, 
$\left.\mathrm{CHCl}_{3}\right)\left(83 \%\right.$ ee). HRMS (ESI) for $\mathrm{C}_{21} \mathrm{H}_{26} \mathrm{BrClNO}_{3} \mathrm{PNa}\left([\mathrm{M}+\mathrm{Na}]^{+}\right)$: calcd 508.0414, found 508.0425 .
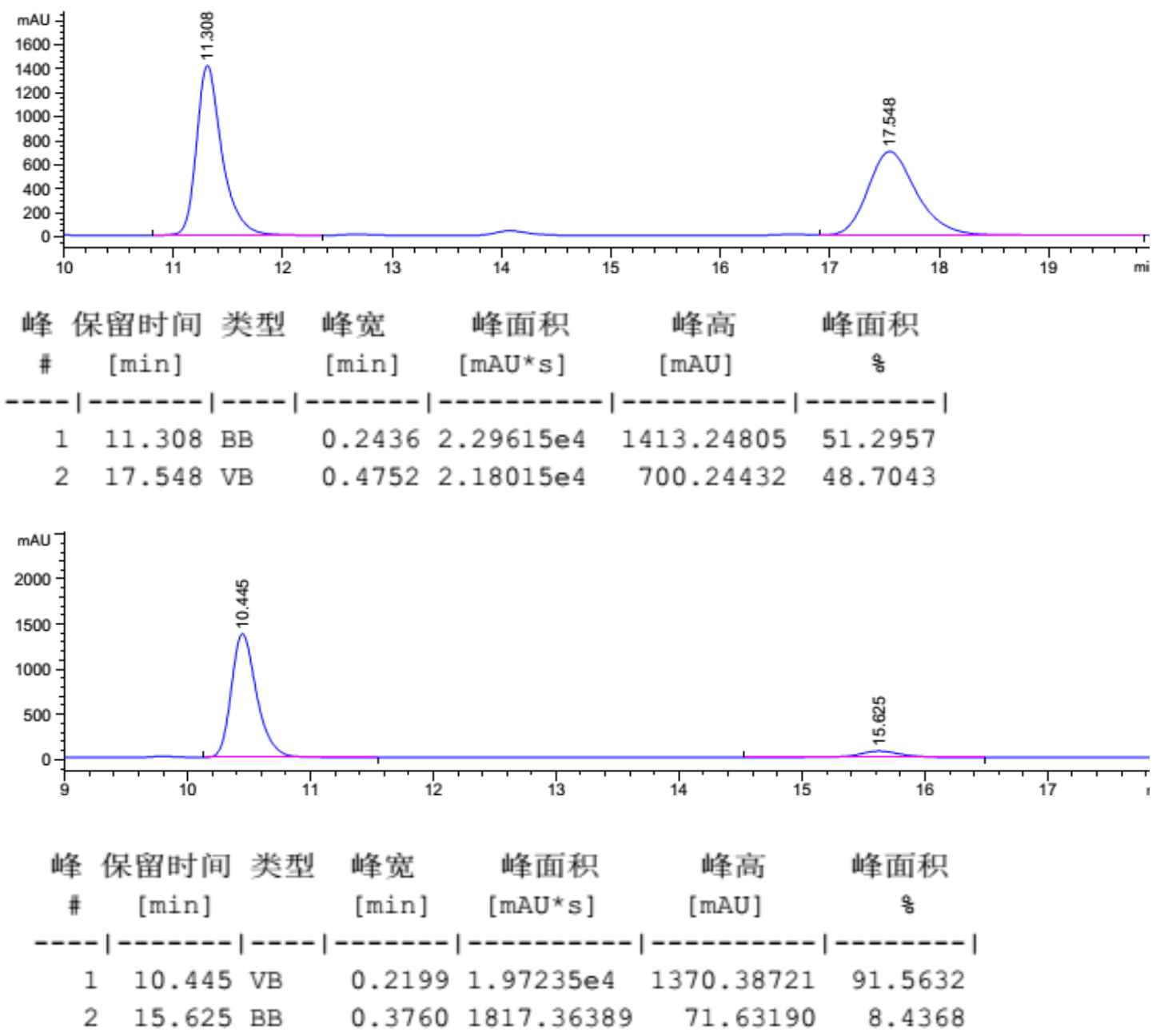<smiles>Cc1ccc(/C=C/[C@H](Nc2ccc(Br)cc2)P(=O)(O)O)cc1</smiles>

(R, E)-diisopropyl (1-((4-bromophenyl)amino)-3-(p-tolyl)allyl)phosphonate

4m: $86 \%$ yield $\left(40 \mathrm{mg}, \mathrm{n}\right.$-pentane/EtOAc $=4: 1$ as the eluent). ${ }^{1} \mathbf{H} \mathbf{N M R}\left(\mathbf{4 0 0} \mathbf{~ M H z}, \mathbf{C D C l}_{\mathbf{3}}\right) \delta 7.30-$ $7.17(\mathrm{~m}, 4 \mathrm{H}), 7.10(\mathrm{~d}, J=7.9 \mathrm{~Hz}, 2 \mathrm{H}), 6.68-6.44(\mathrm{~m}, 3 \mathrm{H}), 6.17(\mathrm{dt}, J=16.1,5.2 \mathrm{~Hz}, 1 \mathrm{H}), 4.74(\mathrm{~m}$, 2H), $4.47-4.13(\mathrm{~m}, 2 \mathrm{H}), 2.32(\mathrm{~s}, 3 \mathrm{H}), 1.34(\mathrm{dd}, J=6.2,1.8 \mathrm{~Hz}, 6 \mathrm{H}), 1.26(\mathrm{t}, J=6.7 \mathrm{~Hz}, 6 \mathrm{H}) .{ }^{13} \mathbf{C}$ NMR (101 MHz, CDCl $\left.)_{3}\right) 145.73(\mathrm{~d}, J=12.9 \mathrm{~Hz}), 137.73,133.36(\mathrm{~d}, J=3.2 \mathrm{~Hz}), 132.70(\mathrm{~d}, J=12.4$ $\mathrm{Hz}), 131.90,129.23,126.40(\mathrm{~d}, J=1.8 \mathrm{~Hz}), 122.02(\mathrm{~d}, J=4.8 \mathrm{~Hz}), 115.26,109.92,72.06$ (d, $J=7.2$ $\mathrm{Hz}), 71.77(\mathrm{~d}, J=7.4 \mathrm{~Hz}), 54.37(\mathrm{~d}, J=154.4 \mathrm{~Hz}), 24.20(\mathrm{~d}, J=3.3 \mathrm{~Hz}), 24.14(\mathrm{~d}, J=3.4 \mathrm{~Hz}), 23.88$ $(\mathrm{d}, J=5.5 \mathrm{~Hz}), 23.82(\mathrm{~d}, J=5.4 \mathrm{~Hz}), 21.18 .{ }^{31} \mathbf{P}$ NMR $\left(\mathbf{1 6 2} \mathbf{~ M H z}, \mathbf{C D C l}_{\mathbf{3}}\right) \delta 20.03$. Enantiomeric excess is $86 \%$ determined by HPLC analysis: Chiralcel $\mathrm{AD}-\mathrm{H}$ (hexane/ $\mathrm{PrOH}=50 / 50$, flow rate $=0.5$ $\mathrm{mL} / \mathrm{min}, 243.4 \mathrm{~nm}), \mathrm{t}_{\mathrm{r}}$ (major $)=14.542 \mathrm{~min}, \mathrm{t}_{\mathrm{r}}($ minor $)=17.808 \mathrm{~min} .[\alpha]_{\mathrm{D}}^{22.4}+70\left(\mathrm{c}=1.0, \mathrm{CHCl}_{3}\right)(86 \%$ 
ee). HRMS (ESI) for $\mathrm{C}_{22} \mathrm{H}_{29} \mathrm{BrNO}_{3} \mathrm{PNa}\left([\mathrm{M}+\mathrm{Na}]^{+}\right)$: calcd 488.0961, found 488.0972.
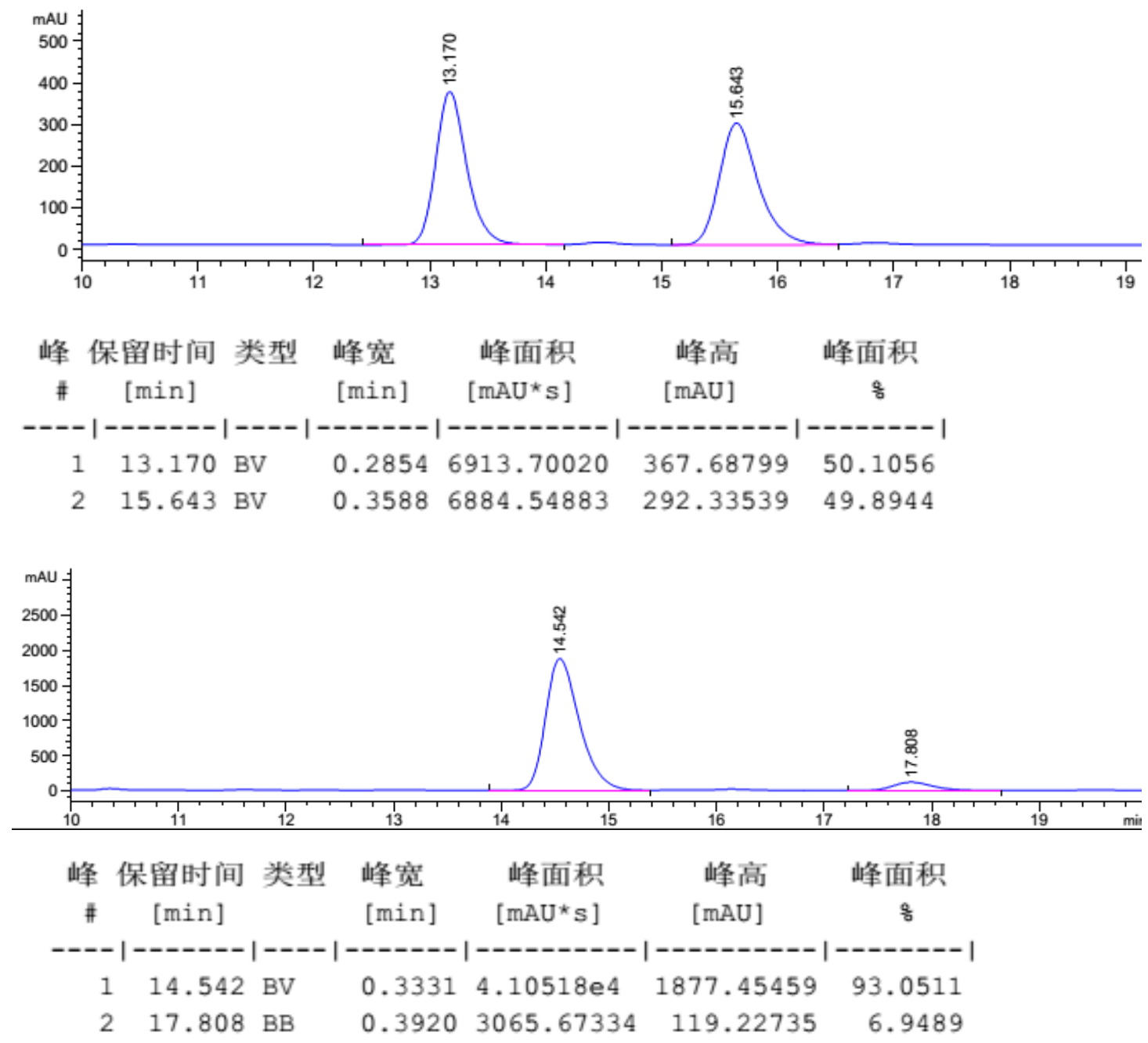<smiles>O=[PH+]C(/C=C/c1ccc(F)cc1)[NH+]([O-])c1ccc(Br)cc1</smiles>

( $R, E$ )-diisopropyl (1-((4-bromophenyl)amino)-3-(4-fluorophenyl)allyl)phosphonate

4n: 85\% yield (40 mg, n-pentane/EtOAc $=4: 1$ as the eluent). ${ }^{1} \mathbf{H} \mathbf{~ N M R}\left(\mathbf{4 0 0} \mathbf{~ M H z}, \mathbf{C D C l}_{\mathbf{3}}\right) \delta 7.30(\mathrm{dd}$, $J=8.3,5.6 \mathrm{~Hz}, 2 \mathrm{H}), 7.27-7.21(\mathrm{~m}, 2 \mathrm{H}), 6.98(\mathrm{t}, J=8.7 \mathrm{~Hz}, 2 \mathrm{H}), 6.65-6.53(\mathrm{~m}, 3 \mathrm{H}), 6.16(\mathrm{dt}, J=$ $16.0,5.2 \mathrm{~Hz}, 1 \mathrm{H}), 4.90-4.56(\mathrm{~m}, 2 \mathrm{H}), 4.33(\mathrm{td}, J=12.9,7.4 \mathrm{~Hz}, 2 \mathrm{H}), 1.34(\mathrm{dd}, J=6.1,2.6 \mathrm{~Hz}, 6 \mathrm{H})$, $1.26(\mathrm{dd}, J=9.5,6.2 \mathrm{~Hz}, 6 \mathrm{H}) .{ }^{13} \mathbf{C} \mathbf{N M R}\left(\mathbf{1 0 1} \mathbf{~ M H z}, \mathbf{C D C l}_{3}\right) \delta 162.35(\mathrm{~d}, J=248.2 \mathrm{~Hz}), 145.61(\mathrm{~d}, J=$ $12.7 \mathrm{~Hz}), 132.31,131.93,131.48(\mathrm{~d}, J=12.3 \mathrm{~Hz}), 128.01(\mathrm{~d}, J=8.0 \mathrm{~Hz}), 122.94(\mathrm{~s}), 115.45(\mathrm{~d}, J=$ $21.7 \mathrm{~Hz}), 115.17,109.99,72.13$ (d, $J=7.3 \mathrm{~Hz}), 71.78$ (d, $J=7.3 \mathrm{~Hz}), 54.21$ (d, $J=154.3 \mathrm{~Hz}), 24.19$ (d, $J=3.3 \mathrm{~Hz}), 24.10(\mathrm{~d}, J=3.4 \mathrm{~Hz}), 23.88(\mathrm{~d}, J=5.3 \mathrm{~Hz}), 23.80(\mathrm{~d}, J=5.2 \mathrm{~Hz}) \cdot{ }^{31} \mathbf{P} \mathbf{N M R}(\mathbf{1 6 2} \mathbf{~ M H z}$, $\left.\mathbf{C D C l}_{3}\right) \delta 19.89 .{ }^{19} \mathbf{F}$ NMR (376 $\left.\mathbf{~ M H z}, \mathbf{C D C l}_{3}\right) \delta$-113.93. Enantiomeric excess is $85 \%$ determined by HPLC analysis: Chiralcel AD-H (hexane/ $\mathrm{PrOH}=50 / 50$, flow rate $=0.5 \mathrm{~mL} / \mathrm{min}, 240.4 \mathrm{~nm}$ ), $\mathrm{t}_{\mathrm{r}}$ (major) $=213.771$ min, $\mathrm{t}_{\mathrm{r}}$ (minor) $=18.670 \mathrm{~min} .[\alpha]_{\mathrm{D}}^{22.5}+75\left(\mathrm{c}=1.0, \mathrm{CHCl}_{3}\right)(85 \%$ ee $)$. HRMS (ESI) for 
$\mathrm{C}_{21} \mathrm{H}_{26} \mathrm{BrFNO}_{3} \mathrm{PNa}\left([\mathrm{M}+\mathrm{Na}]^{+}\right)$: calcd 492.0710 , found 492.0719
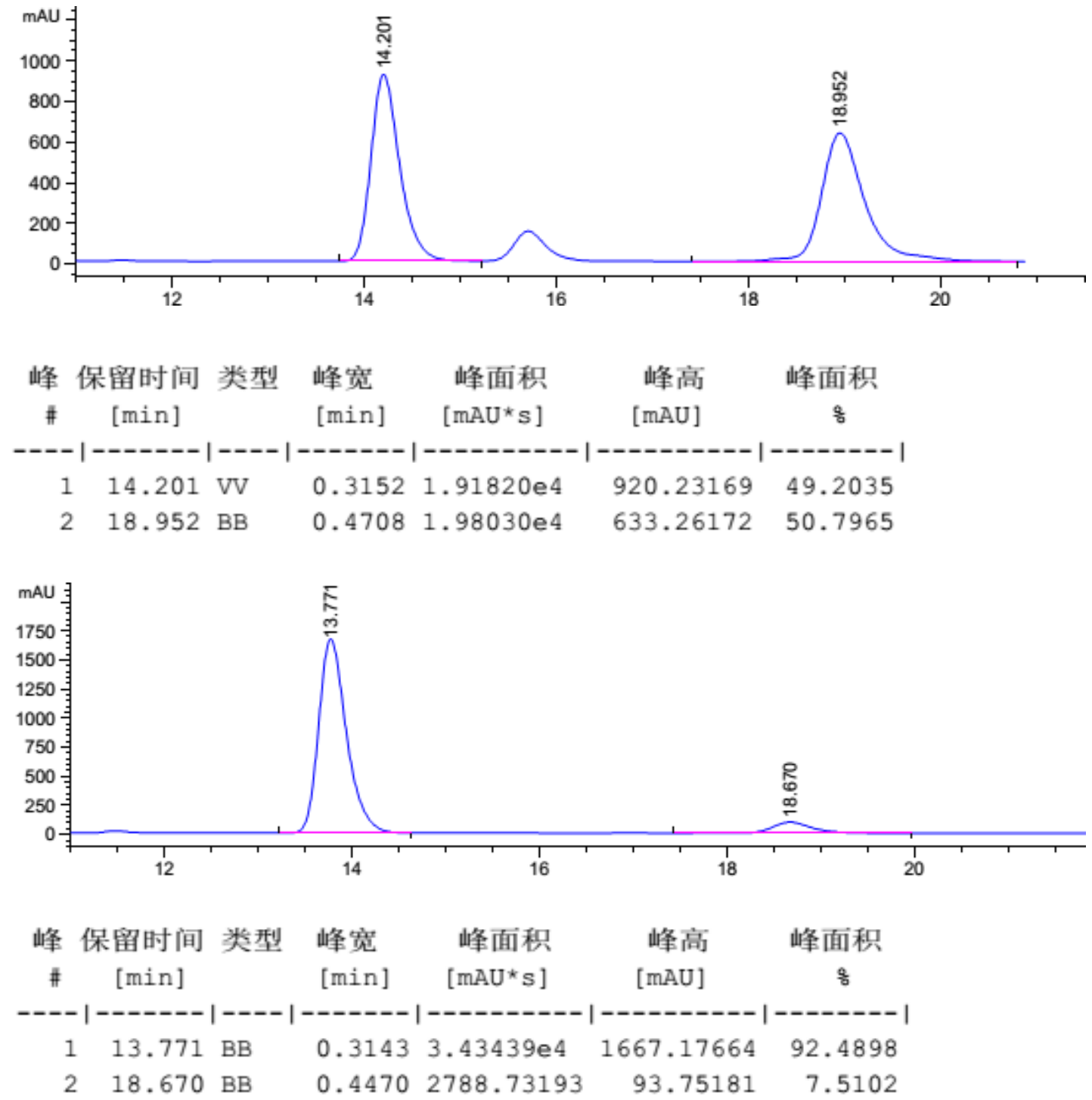<smiles>O=[PH+]C(/C=C/c1ccc(Cl)cc1)[NH+]([O-])c1ccc(Br)cc1</smiles>

(R, E)-diisopropyl (1-((4-bromophenyl)amino)-3-(4-chlorophenyl)allyl)phosphonate

4o: $97 \%$ yield $\left(47 \mathrm{mg}, \mathrm{n}\right.$-pentane/EtOAc $=4: 1$ as the eluent). ${ }^{1} \mathbf{H} \mathbf{~ N M R}\left(\mathbf{4 0 0} \mathbf{~ M H z}, \mathbf{C D C l}_{\mathbf{3}}\right) \delta 7.39-$ $7.05(\mathrm{~m}, 6 \mathrm{H}), 6.76-6.45(\mathrm{~m}, 3 \mathrm{H}), 6.23(\mathrm{dt}, J=17.2,5.2 \mathrm{~Hz}, 1 \mathrm{H}), 4.89-4.55(\mathrm{~m}, 2 \mathrm{H}), 4.51-4.17(\mathrm{~m}$, $2 \mathrm{H}), 1.34(\mathrm{dd}, J=6.1,2.4 \mathrm{~Hz}, 6 \mathrm{H}), 1.25(\mathrm{dd}, J=9.8,6.2 \mathrm{~Hz}, 6 \mathrm{H}) \cdot{ }^{13} \mathbf{C} \mathbf{~ N M R}\left(\mathbf{1 0 1} \mathbf{~ M H z}, \mathbf{C D C l}_{\mathbf{3}}\right) \delta$ $145.56(\mathrm{~d}, J=12.6 \mathrm{~Hz}), 134.60(\mathrm{~d}, J=3.4 \mathrm{~Hz}), 133.43,131.94,131.37$ (d, $J=12.3 \mathrm{~Hz}), 128.68,127.66$ $(\mathrm{d}, J=1.8 \mathrm{~Hz}), 123.96(\mathrm{~d}, J=4.6 \mathrm{~Hz}), 115.15,110.03,72.18(\mathrm{~d}, J=7.2 \mathrm{~Hz}), 71.82(\mathrm{~d}, J=7.3 \mathrm{~Hz})$, $54.22(\mathrm{~d}, J=154.0 \mathrm{~Hz}), 24.18(\mathrm{~d}, J=3.3 \mathrm{~Hz}), 24.09$ (d, $J=3.5 \mathrm{~Hz}), 23.88$ (d, $J=5.2 \mathrm{~Hz}), 23.79$ (d, $J=$ $5.1 \mathrm{~Hz}) .{ }^{31} \mathbf{P}$ NMR $\left(\mathbf{1 6 2} \mathbf{~ M H z}, \mathbf{C D C l}_{\mathbf{3}}\right) \delta$ 19.71. Enantiomeric excess is $85 \%$ determined by HPLC 
analysis: Chiralcel AD-H (hexane/iPrOH $=50 / 50$, flow rate $=0.5 \mathrm{~mL} / \mathrm{min}, 240.4 \mathrm{~nm}), \mathrm{t}_{\mathrm{r}}($ major $)=$ $15.175 \mathrm{~min}, \mathrm{t}_{\mathrm{r}}$ (minor) $=18.361 \mathrm{~min} .[\alpha]_{\mathrm{D}}^{21.7}+97\left(\mathrm{c}=1.0, \mathrm{CHCl}_{3}\right)(85 \%$ ee$)$. HRMS (ESI) for $\mathrm{C}_{21} \mathrm{H}_{26} \mathrm{BrClNO}_{3} \mathrm{PNa}\left([\mathrm{M}+\mathrm{Na}]^{+}\right)$: calcd 508.0414, found 508.0426.
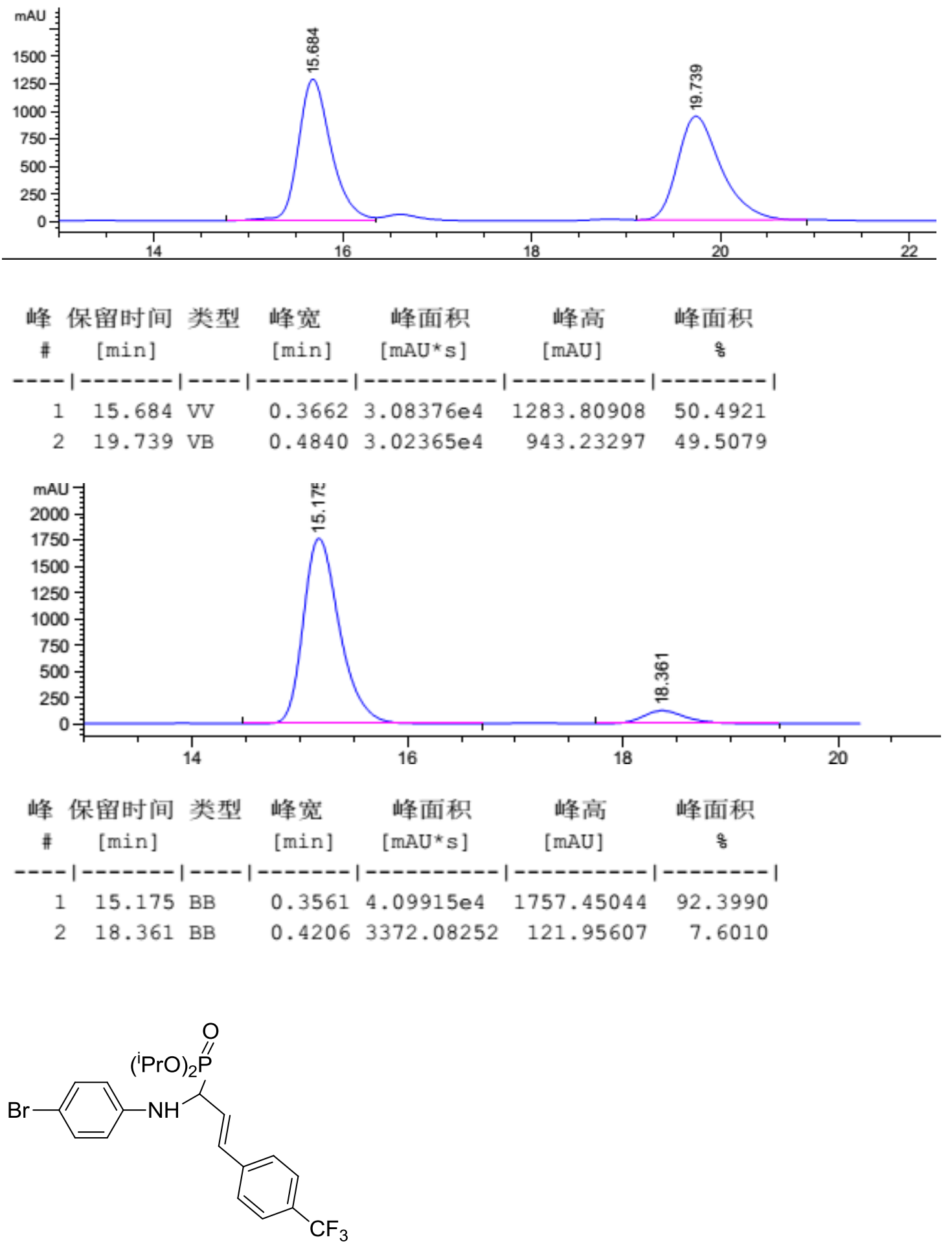

(R, E)-diisopropyl (1-((4-bromophenyl)amino)-3-(4-(trifluoromethyl)phenyl)allyl)phosphonate 4p: $89 \%$ yield $\left(46 \mathrm{mg}, \mathrm{n}\right.$-pentane/EtOAc $=4: 1$ as the eluent). ${ }^{1} \mathbf{H} \mathbf{~ N M R ~}\left(\mathbf{4 0 0} \mathbf{~ M H z}, \mathbf{C D C l}_{\mathbf{3}}\right) \delta 7.63-$ $7.48(\mathrm{~m}, 2 \mathrm{H}), 7.43(\mathrm{~d}, J=8.1 \mathrm{~Hz}, 2 \mathrm{H}), 7.25(\mathrm{~d}, J=8.8 \mathrm{~Hz}, 2 \mathrm{H}), 6.68(\mathrm{dd}, J=16.0,4.8 \mathrm{~Hz}, 1 \mathrm{H}), 6.57(\mathrm{~d}$, $J=8.8 \mathrm{~Hz}, 2 \mathrm{H}), 6.37(\mathrm{dt}, J=15.9,5.0 \mathrm{~Hz}, 1 \mathrm{H}), 4.94-4.64(\mathrm{~m}, 2 \mathrm{H}), 4.55-4.27(\mathrm{~m}, 2 \mathrm{H}), 1.35(\mathrm{dd}, J=$ 6.1, $2.7 \mathrm{~Hz}, 6 \mathrm{H}), 1.26(\mathrm{dd}, J=11.7,6.2 \mathrm{~Hz}, 6 \mathrm{H}) .{ }^{13} \mathbf{C} \mathbf{~ N M R}\left(\mathbf{1 0 1} \mathbf{~ M H z}, \mathbf{C D C l}_{3}\right) \delta 145.50(\mathrm{~d}, J=12.5$ 
$\mathrm{Hz}), 139.55,131.97,131.13(\mathrm{~d}, J=12.1 \mathrm{~Hz}), 129.50$ (q, $J=145.4 \mathrm{~Hz}$ ), 126.60 (d, $J=1.7 \mathrm{~Hz}), 126.21$ $(\mathrm{d}, J=4.6 \mathrm{~Hz}), 125.48(\mathrm{~d}, J=3.7 \mathrm{~Hz}), 124.02(\mathrm{~d}, J=272.0 \mathrm{~Hz}), 115.13,110.12,72.29(\mathrm{~d}, J=7.3 \mathrm{~Hz})$, 71.89 (d, $J=7.3 \mathrm{~Hz}), 54.24$ (d, $J=153.6 \mathrm{~Hz}), 24.16(\mathrm{~d}, J=3.3 \mathrm{~Hz}), 24.06$ (d, $J=3.6 \mathrm{~Hz}), 23.88(\mathrm{~d}, J=$ $5.2 \mathrm{~Hz}), 23.78(\mathrm{~d}, J=5.1 \mathrm{~Hz}) .{ }^{\mathbf{3 1}} \mathbf{P}$ NMR (162 $\left.\mathbf{~ M H z}, \mathbf{C D C l}_{3}\right) \delta 19.42 .{ }^{\mathbf{1 9}} \mathbf{F} \mathbf{~ N M R}\left(\mathbf{3 7 6} \mathbf{~ M H z}, \mathbf{C D C l}_{\mathbf{3}}\right) \delta$ -62.50. Enantiomeric excess is $84 \%$ determined by HPLC analysis: Chiralcel AD-H (hexane/iPrOH $=50 / 50$, flow rate $=0.5 \mathrm{~mL} / \mathrm{min}, 240.4 \mathrm{~nm}), \mathrm{t}_{\mathrm{r}}($ major $)=16.889 \mathrm{~min}, \mathrm{t}_{\mathrm{r}}($ minor $)=23.354 \mathrm{~min} .[\alpha]_{\mathrm{D}}^{21.3}$ $+60\left(\mathrm{c}=1.0, \mathrm{CHCl}_{3}\right)\left(84 \%\right.$ ee). HRMS (ESI) for $\mathrm{C}_{22} \mathrm{H}_{26} \mathrm{BrF}_{3} \mathrm{NO}_{3} \mathrm{PNa}\left([\mathrm{M}+\mathrm{Na}]^{+}\right)$: calcd 542.0678, found 542.0690 .
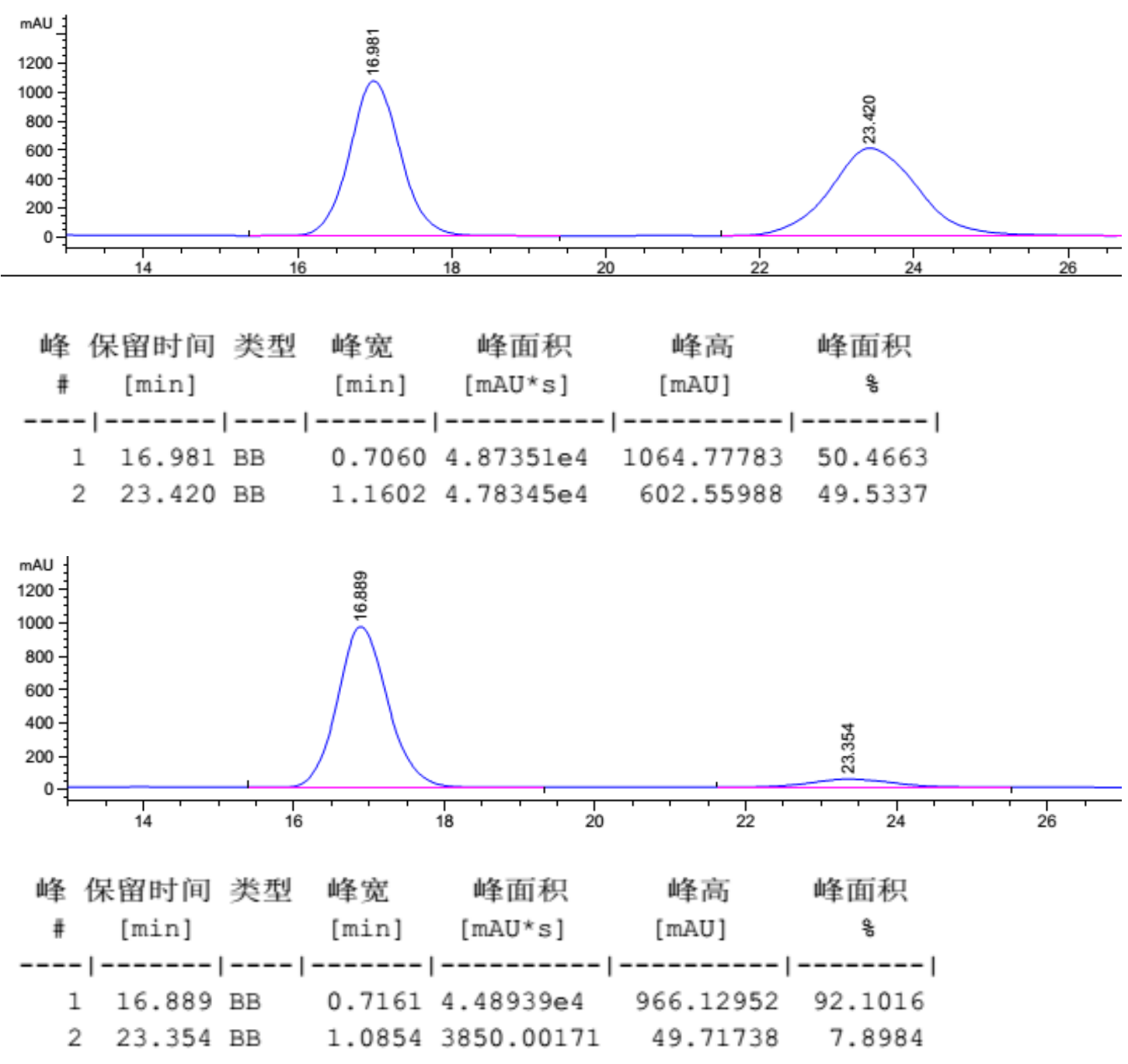<smiles>O=[PH+]([O-])C(/C=C/c1ccc(Br)cc1F)Nc1ccc(Br)cc1</smiles>

(R, E)-diisopropyl (3-(4-bromo-2-fluorophenyl)-1-((4-bromophenyl)amino)allyl)phosphonate

4q: $70 \%$ yield (38 mg, n-pentane/EtOAc $=4: 1$ as the eluent). $\left.{ }^{\mathbf{1}} \mathbf{H} \mathbf{~ N M R ~ ( 4 0 0 ~} \mathbf{~ M H z}, \mathbf{C D C l}_{\mathbf{3}}\right) \delta 7.39-$ $7.04(\mathrm{~m}, 5 \mathrm{H}), 6.84-6.66(\mathrm{~m}, 1 \mathrm{H}), 6.67-6.46(\mathrm{~m}, 2 \mathrm{H}), 6.36(\mathrm{dd}, J=16.1,5.8 \mathrm{~Hz}, 1 \mathrm{H}), 4.89-4.62(\mathrm{~m}$, 
2H), $4.53-4.08(\mathrm{~m}, 2 \mathrm{H}), 1.34$ (dd, $J=6.2,2.4 \mathrm{~Hz}, 6 \mathrm{H}), 1.26(\mathrm{dd}, J=9.7,6.2 \mathrm{~Hz}, 6 \mathrm{H}) .{ }^{13} \mathbf{C}$ NMR (101 MHz, $\left.\mathbf{C D C l}_{3}\right) \delta 159.69(\mathrm{~d}, J=256.5 \mathrm{~Hz}), 145.50(\mathrm{~d}, J=12.6 \mathrm{~Hz}), 131.98,128.63,127.45(\mathrm{~d}, J=3.4$ $\mathrm{Hz}), 126.95,124.32(\mathrm{~d}, J=9.5 \mathrm{~Hz}), 123.17(\mathrm{~d}, J=15.6 \mathrm{~Hz}), 121.32(\mathrm{~d}, J=9.7 \mathrm{~Hz}), 119.34(\mathrm{~d}, J=25.2$ $\mathrm{Hz}), 115.21,110.17,72.24$ (d, $J=7.3 \mathrm{~Hz}), 71.88$ (d, $J=7.4 \mathrm{~Hz}), 54.56$ (d, $J=153.7 \mathrm{~Hz}), 24.16$ (d, $J=$ $3.3 \mathrm{~Hz}), 24.08(\mathrm{~d}, J=3.5 \mathrm{~Hz}), 23.81(\mathrm{~d}, J=5.6 \mathrm{~Hz}), 23.76(\mathrm{~d}, J=5.4 \mathrm{~Hz}) .{ }^{31} \mathbf{P}$ NMR $(\mathbf{1 6 2} \mathbf{~ M H z}$, $\left.\mathbf{C D C l}_{3}\right) \delta$ 19.34. ${ }^{19} \mathbf{F}$ NMR (376 $\left.\mathbf{~ M H z}, \mathbf{C D C l}_{\mathbf{3}}\right) \delta-114.90$. Enantiomeric excess is $87 \%$ determined by HPLC analysis: Chiralcel AD-H (hexane/ $\mathrm{PrOH}=50 / 50$, flow rate $=0.5 \mathrm{~mL} / \mathrm{min}, 240.4 \mathrm{~nm}$ ), $\mathrm{t}_{\mathrm{r}}$ (major) $=14.231 \mathrm{~min}, \mathrm{t}_{\mathrm{r}}($ minor $)=18.842 \mathrm{~min} .[\alpha]_{\mathrm{D}}^{22.5}+62\left(\mathrm{c}=1.0, \mathrm{CHCl}_{3}\right)(87 \%$ ee $)$. HRMS (ESI) for $\mathrm{C}_{21} \mathrm{H}_{25} \mathrm{Br}_{2} \mathrm{FNO}_{3} \mathrm{PNa}\left([\mathrm{M}+\mathrm{Na}]^{+}\right)$: calcd 569.9815 , found 569.9827 .
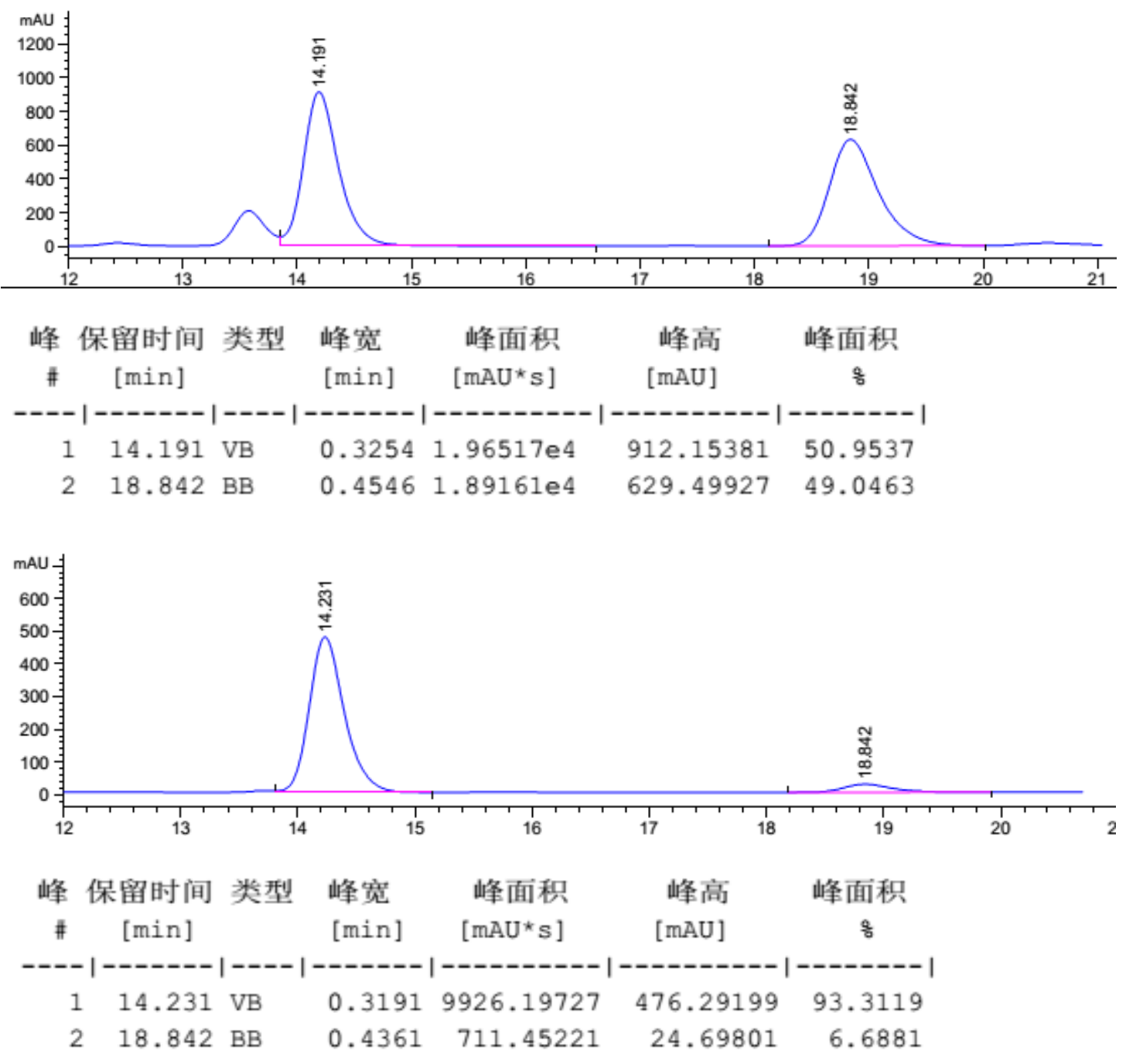<smiles>O=[PH+](O)C(/C=C/c1cc(Br)ccc1F)[NH+]([O-])c1ccc(Br)cc1</smiles>

( $R, E$ )-diisopropyl (3-(5-bromo-2-fluorophenyl)-1-((4-bromophenyl)amino)allyl)phosphonate 4r: $79 \%$ yield (43 mg, n-pentane/EtOAc $=4: 1$ as the eluent). ${ }^{1} \mathbf{H}$ NMR $\left(\mathbf{4 0 0} \mathbf{~ M H z}, \mathbf{C D C l}_{\mathbf{3}}\right) \delta 7.51(\mathrm{dd}$, 
$J=6.6,2.3 \mathrm{~Hz}, 1 \mathrm{H}), 7.39-7.15(\mathrm{~m}, 3 \mathrm{H}), 6.90(\mathrm{dd}, J=10.1,8.8 \mathrm{~Hz}, 1 \mathrm{H}), 6.80-6.64(\mathrm{~m}, 1 \mathrm{H}), 6.64-$ $6.47(\mathrm{~m}, 2 \mathrm{H}), 6.36(\mathrm{dt}, J=16.1,5.1 \mathrm{~Hz}, 1 \mathrm{H}), 4.87-4.66(\mathrm{~m}, 2 \mathrm{H}), 4.52-4.20(\mathrm{~m}, 2 \mathrm{H}), 1.35(\mathrm{dd}, J=$ 6.2, 3.4 Hz, 6H), $1.27(\mathrm{dd}, J=12.7,6.2 \mathrm{~Hz}, 6 \mathrm{H}) .{ }^{13} \mathbf{C}$ NMR (101 MHz, $\left.\mathbf{C D C l}_{3}\right) \delta 157.76,145.45(\mathrm{~d}, J=$ $12.6 \mathrm{~Hz}), 131.95,131.67(\mathrm{~d}, J=8.1 \mathrm{~Hz}), 130.25,127.75,126.00(\mathrm{~d}, J=10.2 \mathrm{~Hz}), 123.91,117.49(\mathrm{~d}, J$ $=23.9 \mathrm{~Hz}), 116.66,115.16,110.14,72.28(\mathrm{~d}, J=7.3 \mathrm{~Hz}), 71.90(\mathrm{~d}, J=7.3 \mathrm{~Hz}), 54.51(\mathrm{~d}, J=153.6 \mathrm{~Hz})$, $24.17(\mathrm{~d}, J=3.3 \mathrm{~Hz}), 24.07(\mathrm{~d}, J=3.5 \mathrm{~Hz}), 23.83(\mathrm{~d}, J=5.4 \mathrm{~Hz}), 23.77(\mathrm{~d}, J=6.3 \mathrm{~Hz}) .{ }^{31} \mathbf{P ~ N M R}(\mathbf{1 6 2}$ MHz, $\left.\mathbf{C D C l}_{3}\right) \delta$ 19.29. ${ }^{19} \mathbf{F}$ NMR (376 $\left.\mathbf{~ M H z , ~} \mathbf{C D C l}_{3}\right) \delta-119.63$. Enantiomeric excess is $81 \%$ determined by HPLC analysis: Chiralcel AD-H (hexane/iPrOH $=50 / 50$, flow rate $=0.5 \mathrm{~mL} / \mathrm{min}, 240.4$ $\mathrm{nm}), \mathrm{t}_{\mathrm{r}}$ (major) $=10.089 \min , \mathrm{t}_{\mathrm{r}}($ minor $)=13.498 \mathrm{~min} .[\alpha]_{\mathrm{D}}^{21.6}+67\left(\mathrm{c}=1.0, \mathrm{CHCl}_{3}\right)(81 \%$ ee $)$. HRMS (ESI) for $\mathrm{C}_{21} \mathrm{H}_{26} \mathrm{Br}_{2} \mathrm{FNO}_{3} \mathrm{P}\left([\mathrm{M}+\mathrm{H}]^{+}\right)$: calcd 547.9996, found 548.0003.
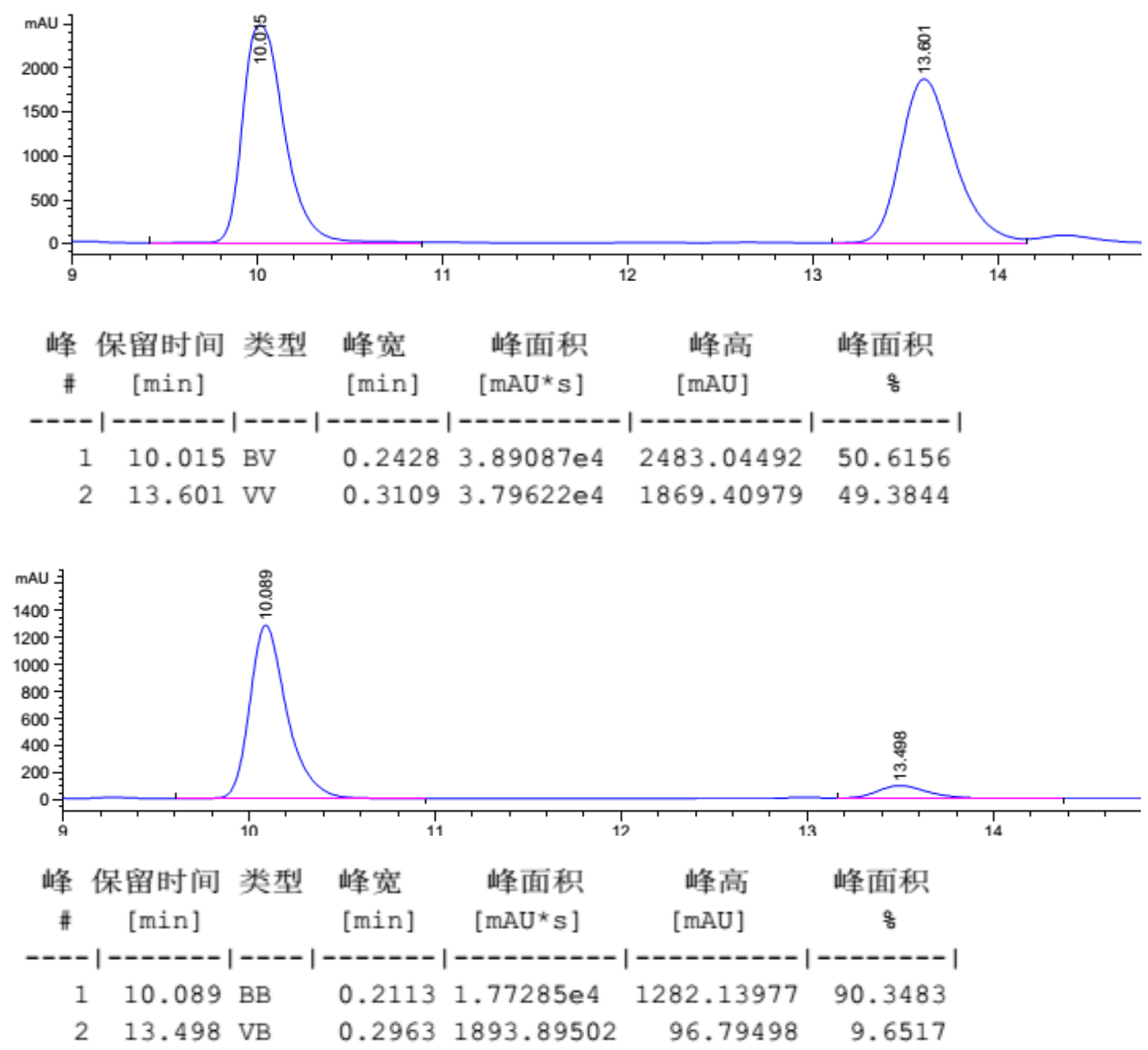<smiles>O=[PH+]([O-])C(/C=C/c1ccc2ccccc2c1)Nc1ccc(Br)cc1</smiles>

(R, E)-diisopropyl (1-((4-bromophenyl)amino)-3-(naphthalen-2-yl)allyl)phosphonate 4s: $59 \%$ yield $\left(30 \mathrm{mg}, \mathrm{n}\right.$-pentane/EtOAc $=4: 1$ as the eluent). ${ }^{\mathbf{1}} \mathbf{H}$ NMR (400 $\left.\mathbf{M H z}, \mathbf{C D C l}_{\mathbf{3}}\right) \delta 7.87-$ 
$7.73(\mathrm{~m}, 3 \mathrm{H}), 7.69(\mathrm{~s}, 1 \mathrm{H}), 7.57(\mathrm{dd}, J=8.6,1.2 \mathrm{~Hz}, 1 \mathrm{H}), 7.51-7.37(\mathrm{~m}, 2 \mathrm{H}), 7.25(\mathrm{dd}, J=8.2,3.7 \mathrm{~Hz}$, $2 \mathrm{H}), 6.81(\mathrm{dd}, J=15.9,4.9 \mathrm{~Hz}, 1 \mathrm{H}), 6.60(\mathrm{~d}, J=8.8 \mathrm{~Hz}, 2 \mathrm{H}), 6.37(\mathrm{dt}, J=16.0,5.3 \mathrm{~Hz}, 1 \mathrm{H}), 4.90-$ $4.53(\mathrm{~m}, 2 \mathrm{H}), 4.60-3.97(\mathrm{~m}, 2 \mathrm{H}), 1.35(\mathrm{dd}, J=6.2,1.3 \mathrm{~Hz}, 6 \mathrm{H}), 1.27(\mathrm{t}, J=6.5 \mathrm{~Hz}, 6 \mathrm{H}) .{ }^{13} \mathbf{C} \mathbf{N M R}$ $\left(\mathbf{1 0 1} \mathbf{M H z}, \mathbf{C D C l}_{3}\right) \delta 145.71(\mathrm{~d}, J=12.8 \mathrm{~Hz}), 133.55,133.42,132.88(\mathrm{t}, J=24.2 \mathrm{~Hz}), 127.96,127.60$, 126.69, 126.67, 126.68 (d, $J=2.5 \mathrm{~Hz}), 126.30,126.00,123.53$ (d, $J=4.7 \mathrm{~Hz}), 123.39,115.25,110.01$, $72.17(\mathrm{~d}, J=7.3 \mathrm{~Hz}), 71.86(\mathrm{~d}, J=7.3 \mathrm{~Hz}), 54.46$ (d, $J=154.2 \mathrm{~Hz}), 24.21$ (d, $J=3.3 \mathrm{~Hz}), 24.14(\mathrm{~d}, J=$ $3.4 \mathrm{~Hz}), 23.89(\mathrm{~d}, J=5.5 \mathrm{~Hz}), 23.84(\mathrm{~d}, J=6.0 \mathrm{~Hz}) .{ }^{31} \mathbf{P}$ NMR (162 $\left.\mathbf{M H z}, \mathbf{C D C l}_{3}\right) \delta 19.95$. Enantiomeric excess is $84 \%$ determined by HPLC analysis: Chiralcel AD-H (hexane/iPrOH $=50 / 50$, flow rate $=0.5 \mathrm{~mL} / \mathrm{min}, 240.4 \mathrm{~nm}), \mathrm{t}_{\mathrm{r}}($ major $)=14.334 \mathrm{~min}, \mathrm{t}_{\mathrm{r}}($ minor $)=20.401 \mathrm{~min} .[\alpha]_{\mathrm{D}}^{20.6}+82$ $\left(\mathrm{c}=1.0, \mathrm{CHCl}_{3}\right)\left(84 \%\right.$ ee). HRMS (ESI) for $\mathrm{C}_{25} \mathrm{H}_{29} \mathrm{BrNO}_{3} \mathrm{PNa}\left([\mathrm{M}+\mathrm{Na}]^{+}\right)$: calcd 524.0961, found 524.0972 .
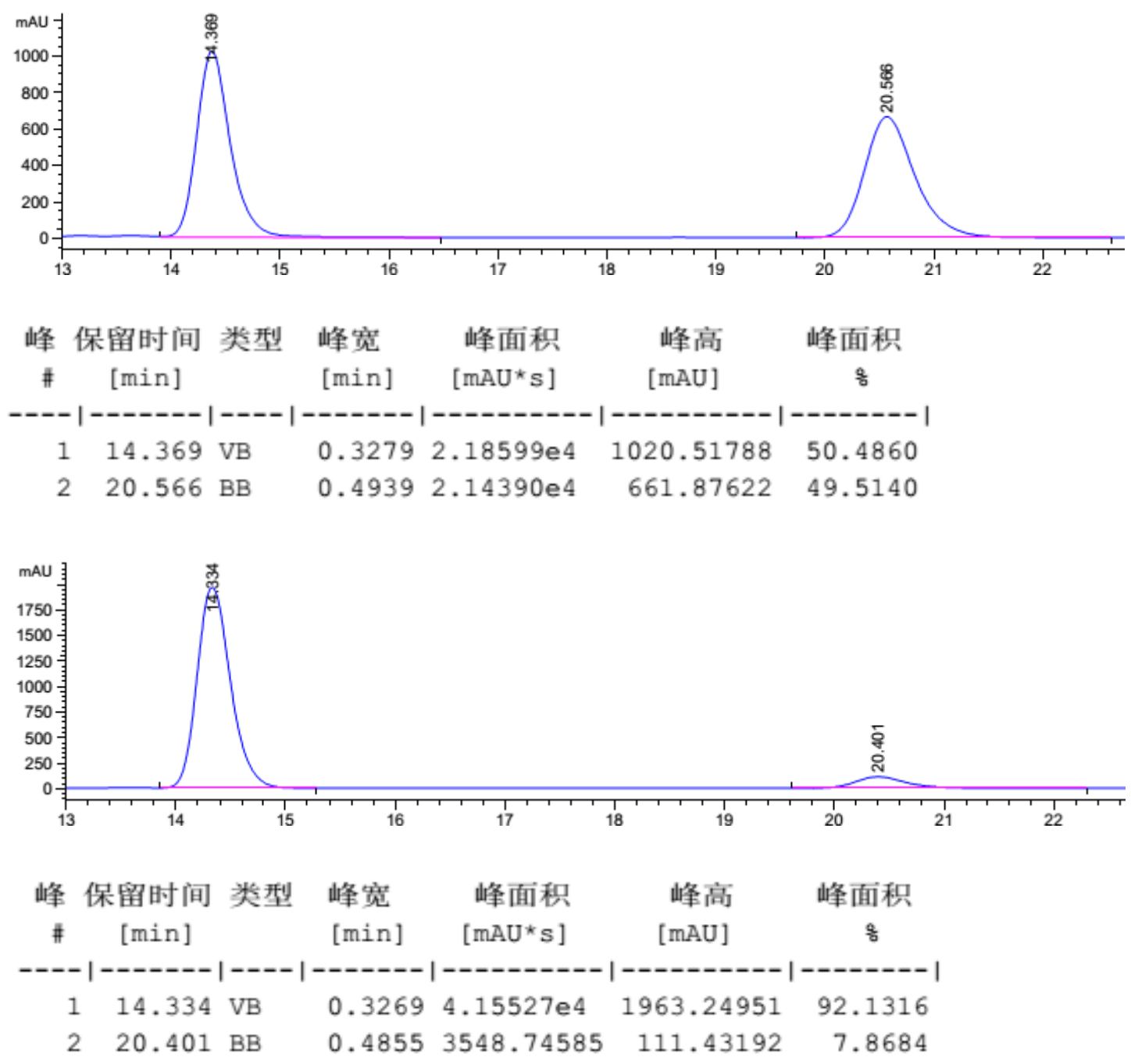
<smiles>CCCCO[PH](=O)[P+](=O)C(/C=C/c1cccc2ccccc12)Nc1ccc(Br)cc1</smiles>

( $R, E)$-diisopropyl (1-((4-bromophenyl)amino)-3-(naphthalen-1-yl)allyl)phosphonate

4t: $64 \%$ yield (32 mg, n-pentane/EtOAc $=4: 1$ as the eluent). ${ }^{1} \mathbf{H}$ NMR $\left(\mathbf{4 0 0} \mathbf{~ M H z}, \mathbf{C D C l}_{\mathbf{3}}\right) \delta 7.92-$ $7.85(\mathrm{~m}, 1 \mathrm{H}), 7.85-7.78(\mathrm{~m}, 1 \mathrm{H}), 7.76(\mathrm{~d}, J=8.1 \mathrm{~Hz}, 1 \mathrm{H}), 7.53(\mathrm{~d}, J=6.9 \mathrm{~Hz}, 1 \mathrm{H}), 7.48-7.34(\mathrm{~m}$, 4H), $7.33-7.21(\mathrm{~m}, 2 \mathrm{H}), 6.65(\mathrm{~d}, J=8.9 \mathrm{~Hz}, 2 \mathrm{H}), 6.26(\mathrm{dd}, J=15.7,5.7 \mathrm{~Hz}, 1 \mathrm{H}), 4.89-4.71(\mathrm{~m}, 2 \mathrm{H})$, $4.45(\mathrm{~d}, J=24.9 \mathrm{~Hz}, 2 \mathrm{H}), 1.36(\mathrm{~d}, J=6.1 \mathrm{~Hz}, 6 \mathrm{H}), 1.29(\mathrm{dd}, J=8.9,6.2 \mathrm{~Hz}, 6 \mathrm{H}) .{ }^{13} \mathbf{C}$ NMR $(101 \mathrm{MHz}$, $\left.\mathbf{C D C l}_{3}\right) \delta 145.81(\mathrm{~d}, J=13.0 \mathrm{~Hz}), 134.16(\mathrm{~d}, J=3.2 \mathrm{~Hz}), 133.51,131.99,131.05,130.41(\mathrm{~d}, J=12.2$ $\mathrm{Hz}), 128.42,128.13,126.42(\mathrm{~d}, J=4.7 \mathrm{~Hz}), 126.09,125.78,125.50,123.91(\mathrm{~d}, J=2.9 \mathrm{~Hz}), 123.70$, $115.51,110.16,72.18(\mathrm{~d}, J=7.2 \mathrm{~Hz}), 71.77(\mathrm{~d}, J=7.3 \mathrm{~Hz}), 54.76(\mathrm{~d}, J=154.0 \mathrm{~Hz}), 24.23(\mathrm{~d}, J=3.4$ Hz), $24.13(\mathrm{~d}, J=3.6 \mathrm{~Hz}), 23.98(\mathrm{~d}, J=5.2 \mathrm{~Hz}), 23.87$ (d, $J=5.1 \mathrm{~Hz}) .{ }^{31} \mathbf{P} \mathbf{N M R}\left(\mathbf{1 6 2} \mathbf{~ M H z}, \mathbf{C D C l}_{3}\right) \delta$ 19.91. Enantiomeric excess is $84 \%$ determined by HPLC analysis: Chiralcel AD-H (hexane/iPrOH $=$ $50 / 50$, flow rate $=0.5 \mathrm{~mL} / \mathrm{min}, 240.4 \mathrm{~nm}), \mathrm{t}_{\mathrm{r}}($ major $)=11.189 \mathrm{~min}, \mathrm{t}_{\mathrm{r}}($ minor $)=14.045 \mathrm{~min} .[\alpha]_{\mathrm{D}}{ }^{21.9}+46$ $\left(\mathrm{c}=1.0, \mathrm{CHCl}_{3}\right)\left(84 \%\right.$ ee). HRMS (ESI) for $\mathrm{C}_{25} \mathrm{H}_{30} \mathrm{BrNO}_{3} \mathrm{P}\left([\mathrm{M}+\mathrm{H}]^{+}\right)$: calcd 502.1141, found 502.1151 .
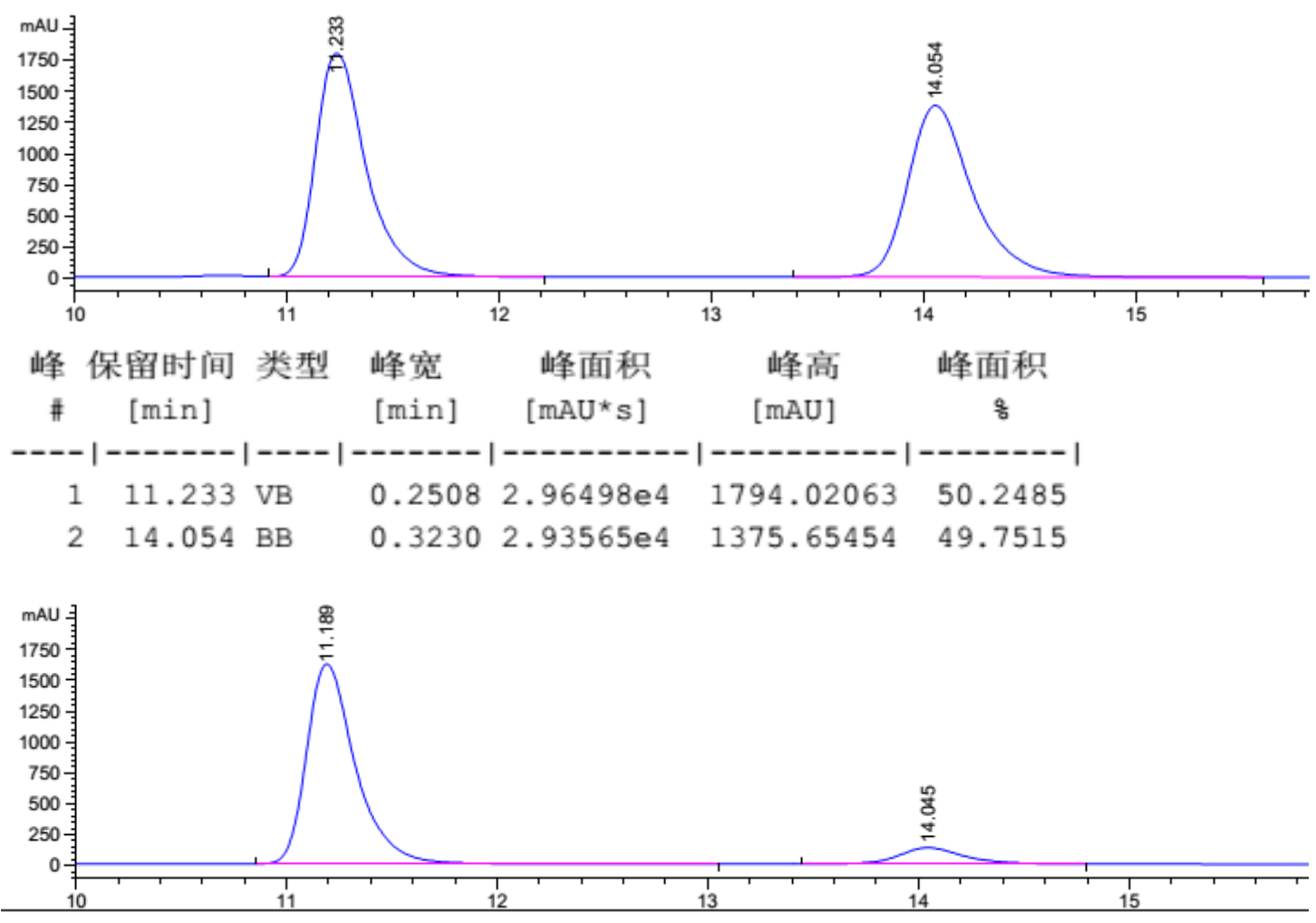


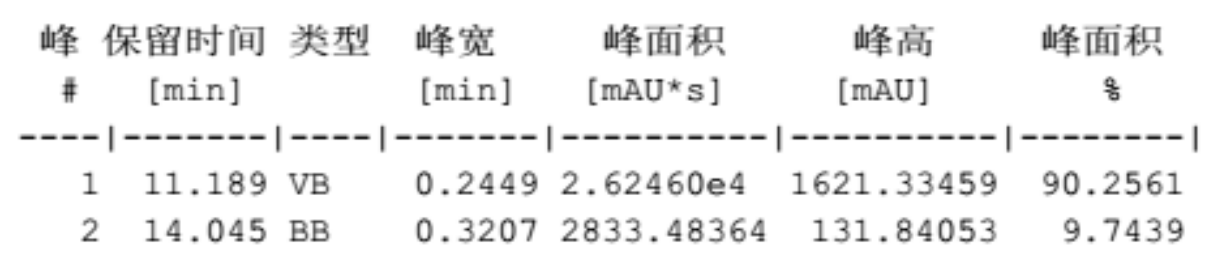<smiles>O=[PH](O)C(/C=C/C=C/c1ccccc1)Nc1ccc(Br)cc1</smiles>

Diisopropyl(( $R, 2 E, 4 E)$-1-((4-bromophenyl)amino)-5-phenylpenta-2,4-dien-1-yl)phosphonate 4u: $37 \%$ yield (18 $\mathrm{mg}, \mathrm{n}$-pentane/EtOAc $=4: 1$ as the eluent). ${ }^{\mathbf{1}} \mathbf{H} \mathbf{~ N M R}\left(\mathbf{4 0 0} \mathbf{~ M H z}, \mathbf{C D C l}_{\mathbf{3}}\right) \delta 7.37(\mathrm{~d}$, $J=7.4 \mathrm{~Hz}, 2 \mathrm{H}), 7.30(\mathrm{t}, J=7.5 \mathrm{~Hz}, 3 \mathrm{H}), 7.23(\mathrm{dd}, J=12.2,5.1 \mathrm{~Hz}, 2 \mathrm{H}), 6.79(\mathrm{dd}, J=15.6,10.7$ $\mathrm{Hz}, 1 \mathrm{H}), 6.64-6.23(\mathrm{~m}, 4 \mathrm{H}), 5.84(\mathrm{dt}, J=15.3,5.4 \mathrm{~Hz}, 1 \mathrm{H}), 4.95-4.39(\mathrm{~m}, 2 \mathrm{H}), 4.51-3.94(\mathrm{~m}$, 2H), 1.35 (dd, $J=6.2,2.1 \mathrm{~Hz}, 6 \mathrm{H}), 1.28(\mathrm{dd}, J=13.5,6.1 \mathrm{~Hz}, 6 \mathrm{H}) .{ }^{13} \mathbf{C} \mathbf{~ N M R}\left(\mathbf{1 0 1} \mathbf{~ M H z}, \mathbf{C D C l}_{3}\right)$ $\delta 145.67(\mathrm{~d}, J=13.1 \mathrm{~Hz}), 136.89,133.29,133.23(\mathrm{~d}, J=4.0 \mathrm{~Hz}), 131.97,128.60,127.71,127.60$ $(\mathrm{d}, J=5.1 \mathrm{~Hz}), 127.05(\mathrm{~d}, J=6.1 \mathrm{~Hz}), 126.39,115.23,110.02,72.15(\mathrm{~d}, J=7.1 \mathrm{~Hz}), 71.81(\mathrm{~d}, J=$ $7.1 \mathrm{~Hz}), 54.68(\mathrm{~d}, J=154.5 \mathrm{~Hz}), 24.23(\mathrm{~d}, J=4.0 \mathrm{~Hz}), 24.15(\mathrm{~d}, J=4.1 \mathrm{~Hz}), 23.90(\mathrm{~d}, J=6.1 \mathrm{~Hz})$, $23.84(\mathrm{~d}, J=6.0 \mathrm{~Hz}) .{ }^{31} \mathbf{P}$ NMR $\left(\mathbf{1 6 2} \mathbf{~ M H z}, \mathbf{C D C l}_{\mathbf{3}}\right) \delta 19.83$. Enantiomeric excess is $77 \%$ determined by HPLC analysis: Chiralcel AD-H (hexane $/ \mathrm{PrOH}=50 / 50$, flow rate $=0.5 \mathrm{~mL} / \mathrm{min}, 240.4$ $\mathrm{nm}), \mathrm{t}_{\mathrm{r}}($ major $)=12.594 \mathrm{~min}, \mathrm{t}_{\mathrm{r}}($ minor $)=19.806 \mathrm{~min} .[\alpha]_{\mathrm{D}}^{22.3}+77\left(\mathrm{c}=1.0, \mathrm{CHCl}_{3}\right)(77 \%$ ee $) . \mathbf{H R M S}$ (ESI) for $\mathrm{C}_{23} \mathrm{H}_{29} \mathrm{BrNO}_{3} \mathrm{PNa}\left([\mathrm{M}+\mathrm{Na}]^{+}\right)$: calcd 500.0961, found 500.0972 .
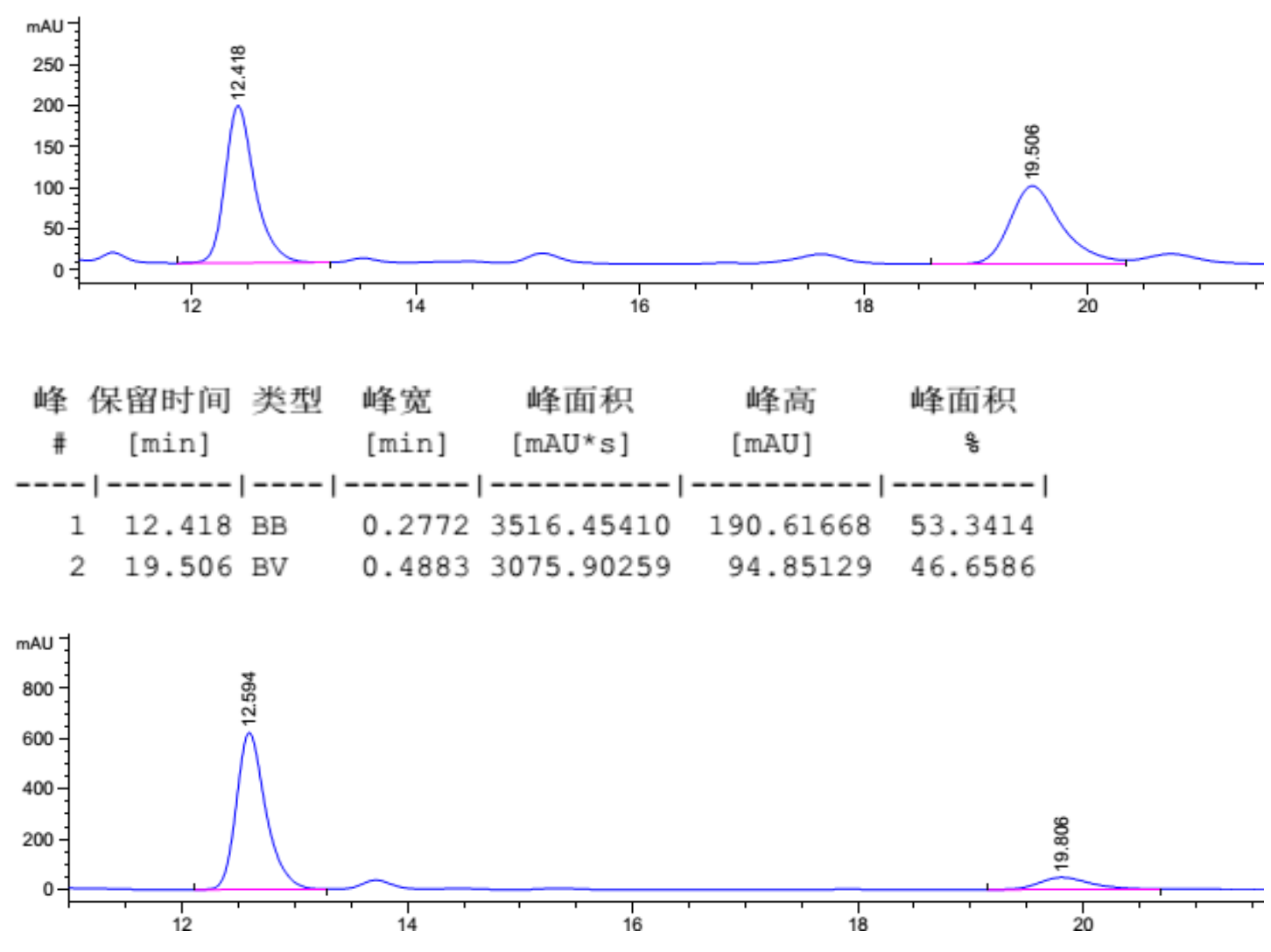


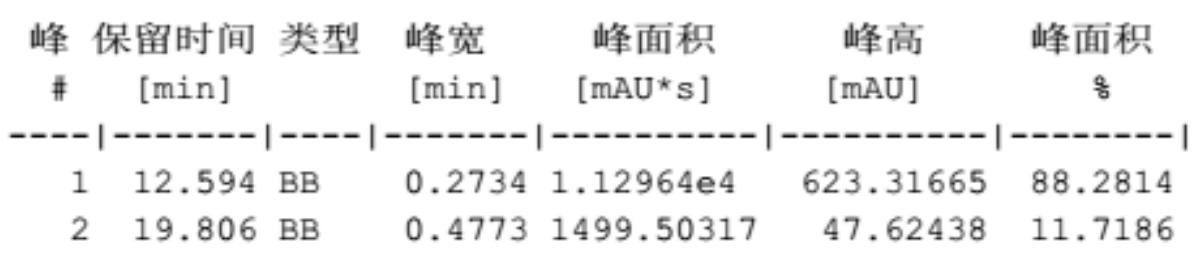<smiles>O=[PH+]([O-])[C@H](/C=C/c1ccco1)Nc1ccc(Br)cc1</smiles>

( $R, E$ )-diisopropyl (1-((4-bromophenyl)amino)-3-(furan-2-yl)allyl)phosphonate

4v: $91 \%$ yield (40 mg, n-pentane/EtOAc $=4: 1$ as the eluent). ${ }^{\mathbf{1}} \mathbf{H} \mathbf{N M R}\left(\mathbf{4 0 0} \mathbf{~ M H z}, \mathbf{C D C l}_{\mathbf{3}}\right) \delta 7.33$ (s, $1 \mathrm{H}), 7.25(\mathrm{dt}, J=5.0,3.8 \mathrm{~Hz}, 2 \mathrm{H}), 6.60-6.50(\mathrm{~m}, 2 \mathrm{H}), 6.50-6.40(\mathrm{~m}, 1 \mathrm{H}), 6.34(\mathrm{dd}, J=3.3,1.8 \mathrm{~Hz}$, $1 \mathrm{H}), 6.25-6.13(\mathrm{~m}, 2 \mathrm{H}), 4.75(\mathrm{~m}, 2 \mathrm{H}), 4.45-4.16(\mathrm{~m}, 2 \mathrm{H}), 1.37-1.31(\mathrm{~m}, 6 \mathrm{H}), 1.27(\mathrm{dd}, J=17.5$, $6.2 \mathrm{~Hz}, 6 \mathrm{H}) .{ }^{13} \mathbf{C}$ NMR (101 MHz, $\left.\mathbf{C D C l}_{\mathbf{3}}\right) \delta 151.98(\mathrm{~d}, J=4.4 \mathrm{~Hz}), 145.55(\mathrm{~d}, J=12.8 \mathrm{~Hz}), 142.18$, 131.93, $121.56(\mathrm{~d}, J=5.1 \mathrm{~Hz}), 120.71(\mathrm{~d}, J=12.4 \mathrm{~Hz}), 115.13,111.27,109.95,108.49(\mathrm{~d}, J=2.8 \mathrm{~Hz})$, $72.12(\mathrm{~d}, J=7.2 \mathrm{~Hz}), 71.88$ (d, $J=7.4 \mathrm{~Hz}), 53.89$ (d, $J=154.4 \mathrm{~Hz}), 24.19(\mathrm{~d}, J=3.4 \mathrm{~Hz}), 24.14$ (d, $J=$ $3.4 \mathrm{~Hz}), 23.83(\mathrm{~d}, J=2.1 \mathrm{~Hz}), 23.78(\mathrm{~d}, J=1.8 \mathrm{~Hz}) .{ }^{31} \mathbf{P}$ NMR $\left(\mathbf{1 6 2} \mathbf{~ M H z}, \mathbf{C D C l}_{\mathbf{3}}\right) \delta 19.70$. Enantiomeric excess is $82 \%$ determined by HPLC analysis: Chiralcel AD-H (hexane/iPrOH $=50 / 50$, flow rate $=0.5 \mathrm{~mL} / \mathrm{min}, 240.4 \mathrm{~nm}), \mathrm{t}_{\mathrm{r}}$ (major) $=15.843 \mathrm{~min}, \mathrm{t}_{\mathrm{r}}$ (minor $)=21.796 \mathrm{~min} .[\alpha]_{\mathrm{D}}^{21.9}+62(\mathrm{c}=1.0$, $\left.\mathrm{CHCl}_{3}\right)\left(82 \%\right.$ ee). HRMS (ESI) for $\mathrm{C}_{19} \mathrm{H}_{25} \mathrm{BrNO}_{4} \mathrm{PNa}\left([\mathrm{M}+\mathrm{Na}]^{+}\right)$: calcd 464.0597, found 464.0604 .
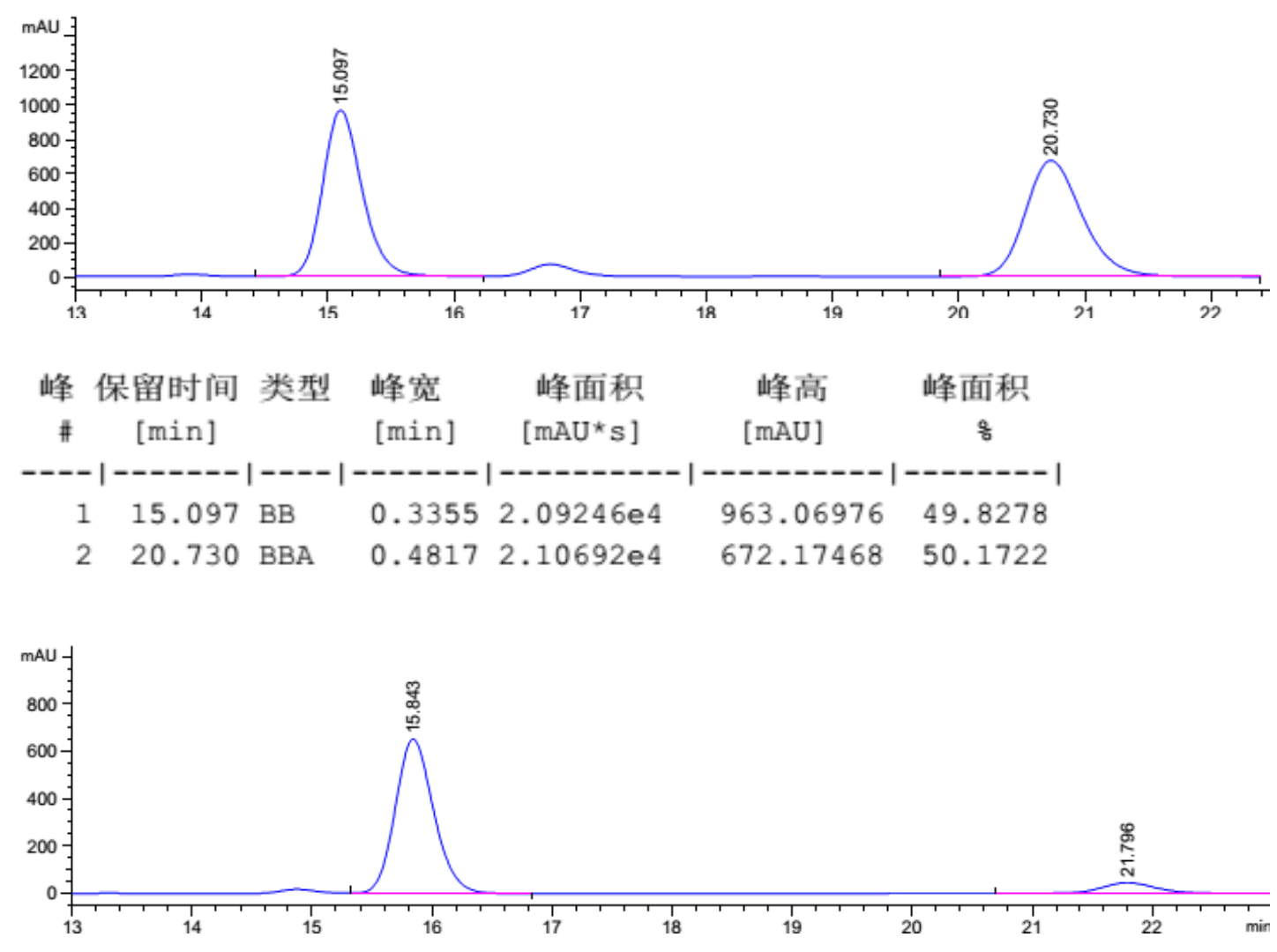


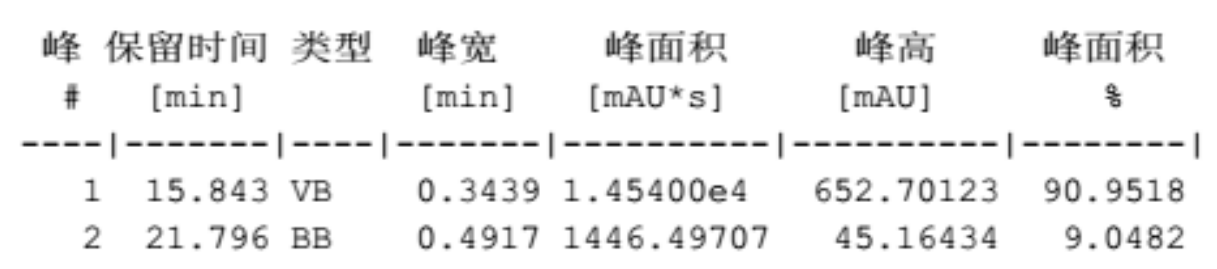<smiles>O=[P+]([C@H](/C=C/c1cccs1)Nc1ccc(Br)cc1)P(=O)(O)O</smiles>

(R, E)-diisopropyl (1-((4-bromophenyl)amino)-3-(thiophen-2-yl)allyl)phosphonate

4w: $90 \%$ yield (41 mg, n-pentane/EtOAc $=4: 1$ as the eluent). ${ }^{1} \mathbf{H}$ NMR $\left(\mathbf{4 0 0} \mathbf{~ M H z}, \mathbf{C D C l}_{\mathbf{3}}\right) \delta 7.24$ $(\mathrm{s}, 2 \mathrm{H}), 7.15(\mathrm{~d}, J=5.0 \mathrm{~Hz}, 1 \mathrm{H}), 6.93(\mathrm{dt}, J=7.8,3.5 \mathrm{~Hz}, 2 \mathrm{H}), 6.77(\mathrm{dd}, J=15.8,4.9 \mathrm{~Hz}, 1 \mathrm{H}), 6.60-$ $6.49(\mathrm{~m}, 2 \mathrm{H}), 6.16-6.02(\mathrm{~m}, 1 \mathrm{H}), 4.94-4.56(\mathrm{~m}, 3 \mathrm{H}), 4.47-4.00(\mathrm{~m}, 2 \mathrm{H}), 1.38-1.32(\mathrm{~m}, 6 \mathrm{H}), 1.27$ $(\mathrm{dd}, J=14.7,6.2 \mathrm{~Hz}, 6 \mathrm{H}) .{ }^{13} \mathbf{C}$ NMR $\left(\mathbf{1 0 1} \mathbf{~ M H z}, \mathbf{C D C l}_{\mathbf{3}}\right) \delta 145.57(\mathrm{~d}, J=12.8 \mathrm{~Hz}), 141.29,131.98$, 127.38, 126.19 (d, $J=3.0 \mathrm{~Hz}), 125.76(\mathrm{~d}, J=12.5 \mathrm{~Hz}), 124.62,122.64$ (d, $J=5.2 \mathrm{~Hz}), 115.18,110.05$, $72.17(\mathrm{~d}, J=7.1 \mathrm{~Hz}), 71.97(\mathrm{~d}, J=7.4 \mathrm{~Hz}), 54.01(\mathrm{~d}, J=154.4 \mathrm{~Hz}), 24.21(\mathrm{~d}, J=3.6 \mathrm{~Hz}), 24.19(\mathrm{~d}, J=$ $3.6 \mathrm{~Hz}), 23.91(\mathrm{~d}, J=5.5 \mathrm{~Hz}), 23.85(\mathrm{~d}, J=5.9 \mathrm{~Hz}) .{ }^{31} \mathbf{P} \mathbf{~ N M R}\left(\mathbf{1 6 2} \mathbf{~ M H z}, \mathbf{C D C l}_{\mathbf{3}}\right) \delta 19.75$. Enantiomeric excess is $81 \%$ determined by HPLC analysis: Chiralcel AD-H (hexane/iPrOH $=50 / 50$, flow rate $=0.5 \mathrm{~mL} / \mathrm{min}, 240.4 \mathrm{~nm}), \mathrm{t}_{\mathrm{r}}$ (major) $=13.755 \mathrm{~min}, \mathrm{t}_{\mathrm{r}}$ (minor) $=20.932 \mathrm{~min} .[\alpha]_{\mathrm{D}}{ }^{21.5}+56$ $\left(\mathrm{c}=1.0, \mathrm{CHCl}_{3}\right)\left(81 \%\right.$ ee). HRMS (ESI) for $\mathrm{C}_{19} \mathrm{H}_{25} \mathrm{BrNO}_{3} \mathrm{PSNa}\left([\mathrm{M}+\mathrm{Na}]^{+}\right)$: calcd 480.0368 , found 480.0378 .
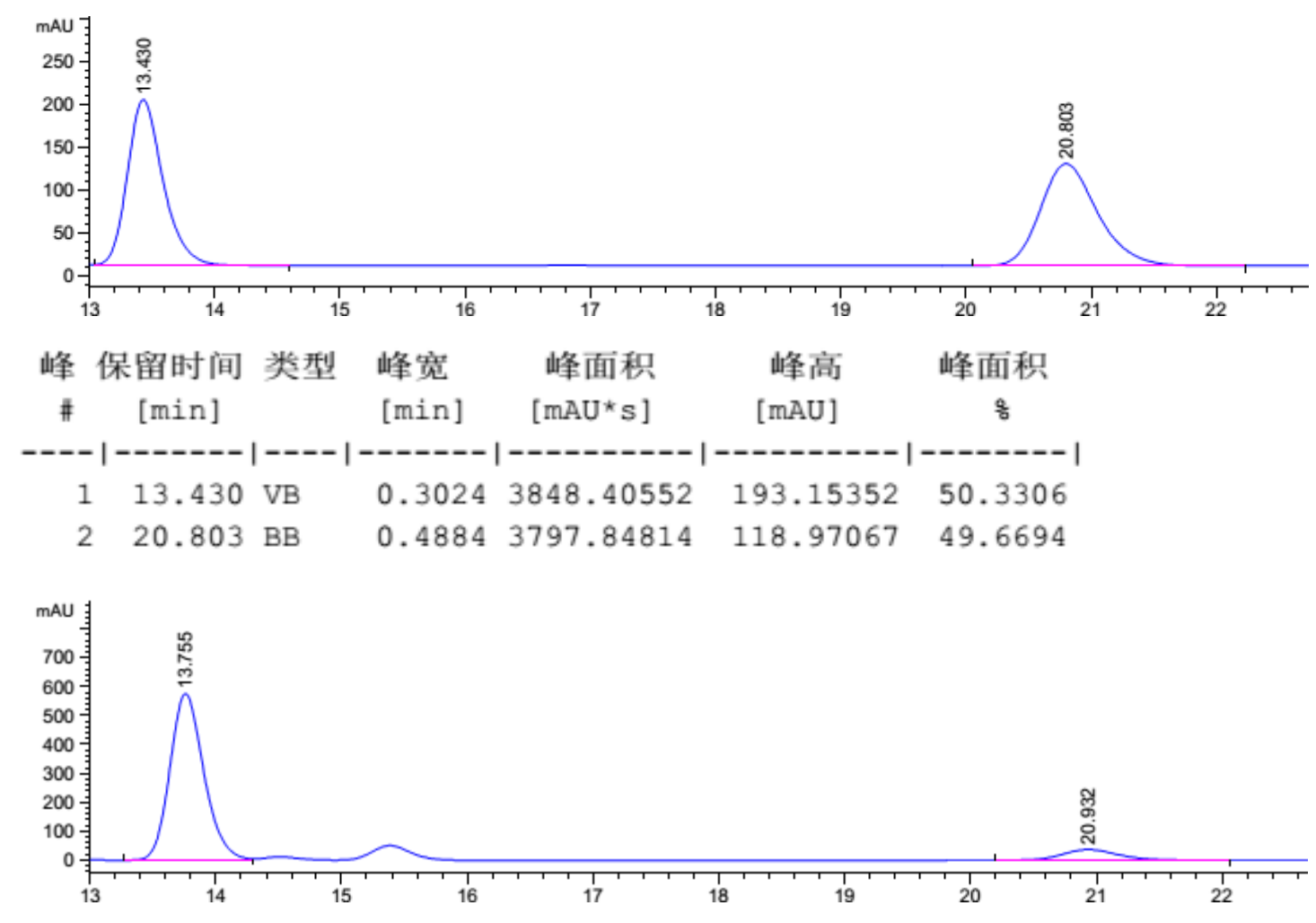

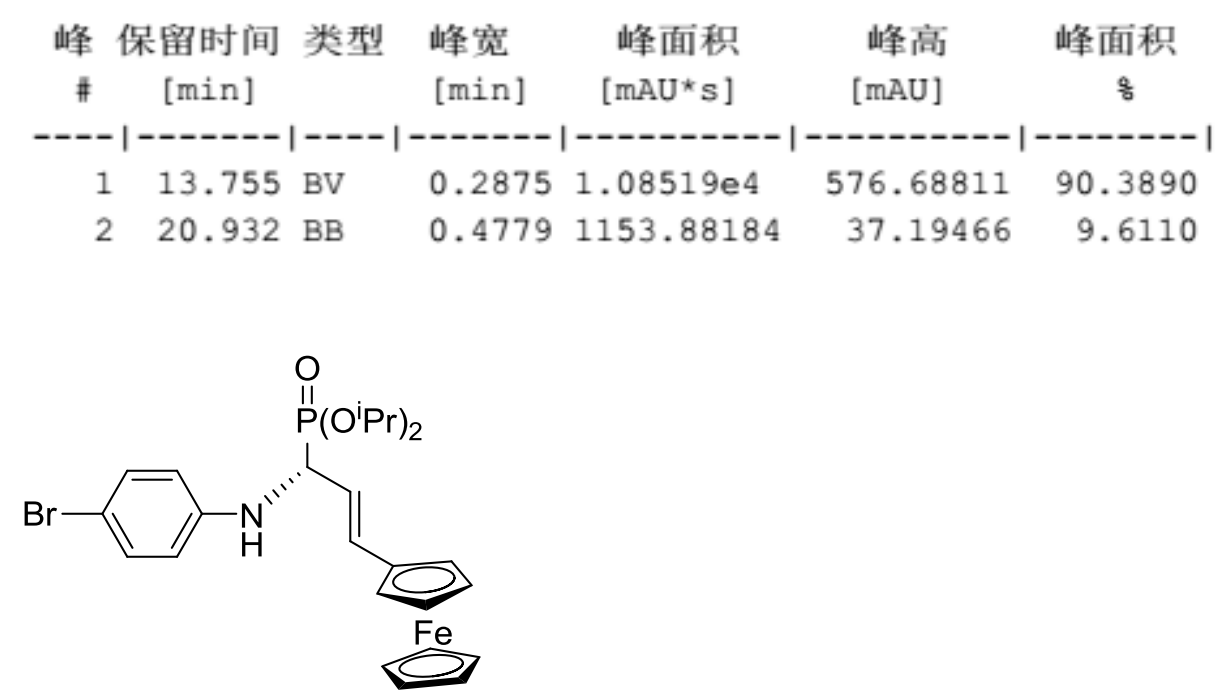

(R, E)-diisopropyl (1-((4-bromophenyl)amino)-3-(ferrocen-2-yl)allyl)phosphonate

4x: 30\% yield (17 mg, n-pentane/EtOAc $=4: 1$ as the eluent). ${ }^{1} \mathbf{H}$ NMR (400 $\left.\mathbf{~ M H z}, \mathbf{C D C l}_{\mathbf{3}}\right) \delta 7.26(\mathrm{t}, J$ $=4.3 \mathrm{~Hz}, 2 \mathrm{H}), 6.58(\mathrm{~d}, J=8.7 \mathrm{~Hz}, 2 \mathrm{H}), 6.39(\mathrm{dd}, J=15.7,4.8 \mathrm{~Hz}, 1 \mathrm{H}), 5.90-5.63(\mathrm{~m}, 1 \mathrm{H}), 4.89-$ $4.60(\mathrm{~m}, 2 \mathrm{H}), 4.31(\mathrm{~d}, J=1.6 \mathrm{~Hz}, 3 \mathrm{H}), 4.21(\mathrm{~d}, J=12.6 \mathrm{~Hz}, 2 \mathrm{H}), 4.16-4.09(\mathrm{~m}, 1 \mathrm{H}), 4.05(\mathrm{~s}, 5 \mathrm{H})$, $1.36(\mathrm{dd}, J=6.1,2.1 \mathrm{~Hz}, 6 \mathrm{H}), 1.31(\mathrm{dd}, J=11.3,6.1 \mathrm{~Hz}, 6 \mathrm{H}) .{ }^{\mathbf{1 3}} \mathbf{C} \mathbf{N M R}\left(\mathbf{1 0 1} \mathbf{M H z}, \mathbf{C D C l}_{3}\right) \delta 145.83$, $(\mathrm{d}, J=13.1 \mathrm{~Hz}), 131.85,130.10(\mathrm{~d}, J=6.1 \mathrm{~Hz}), 115.45,110.01,81.85,71.95(\mathrm{~d}, J=7.1 \mathrm{~Hz}), 71.59(\mathrm{~d}$, $J=7.1 \mathrm{~Hz}), 69.07,68.84,66.88$ (d, $J=14.1 \mathrm{~Hz}), 54.39$ (d, $J=155.5 \mathrm{~Hz}), 24.20$ (d, $J=4.1 \mathrm{~Hz}), 24.01$ $(\mathrm{d}, J=3.1 \mathrm{~Hz}), 23.98(\mathrm{~d}, J=5.5 \mathrm{~Hz}), 23.89$ (d, $J=5.5 \mathrm{~Hz}) .{ }^{31} \mathbf{P}$ NMR (162 MHz, CDCl 3 ) $\delta 20.03$. Enantiomeric excess is $92 \%$ determined by HPLC analysis: Chiralcel AD-H (hexane/iPrOH $=50 / 50$, flow rate $=0.5 \mathrm{~mL} / \mathrm{min}, 240.4 \mathrm{~nm}), \mathrm{t}_{\mathrm{r}}($ major $)=11.095 \mathrm{~min}, \mathrm{t}_{\mathrm{r}}($ minor $)=14.900 \min .[\alpha]_{\mathrm{D}}^{20.9}$ $+107\left(\mathrm{c}=1.0, \mathrm{CHCl}_{3}\right)\left(90 \%\right.$ ee). HRMS (ESI) for $\mathrm{C}_{25} \mathrm{H}_{32} \mathrm{BrFeNO}_{3} \mathrm{P}\left([\mathrm{M}+\mathrm{H}]^{+}\right)$: calcd 560.0602, found 560.0608 .
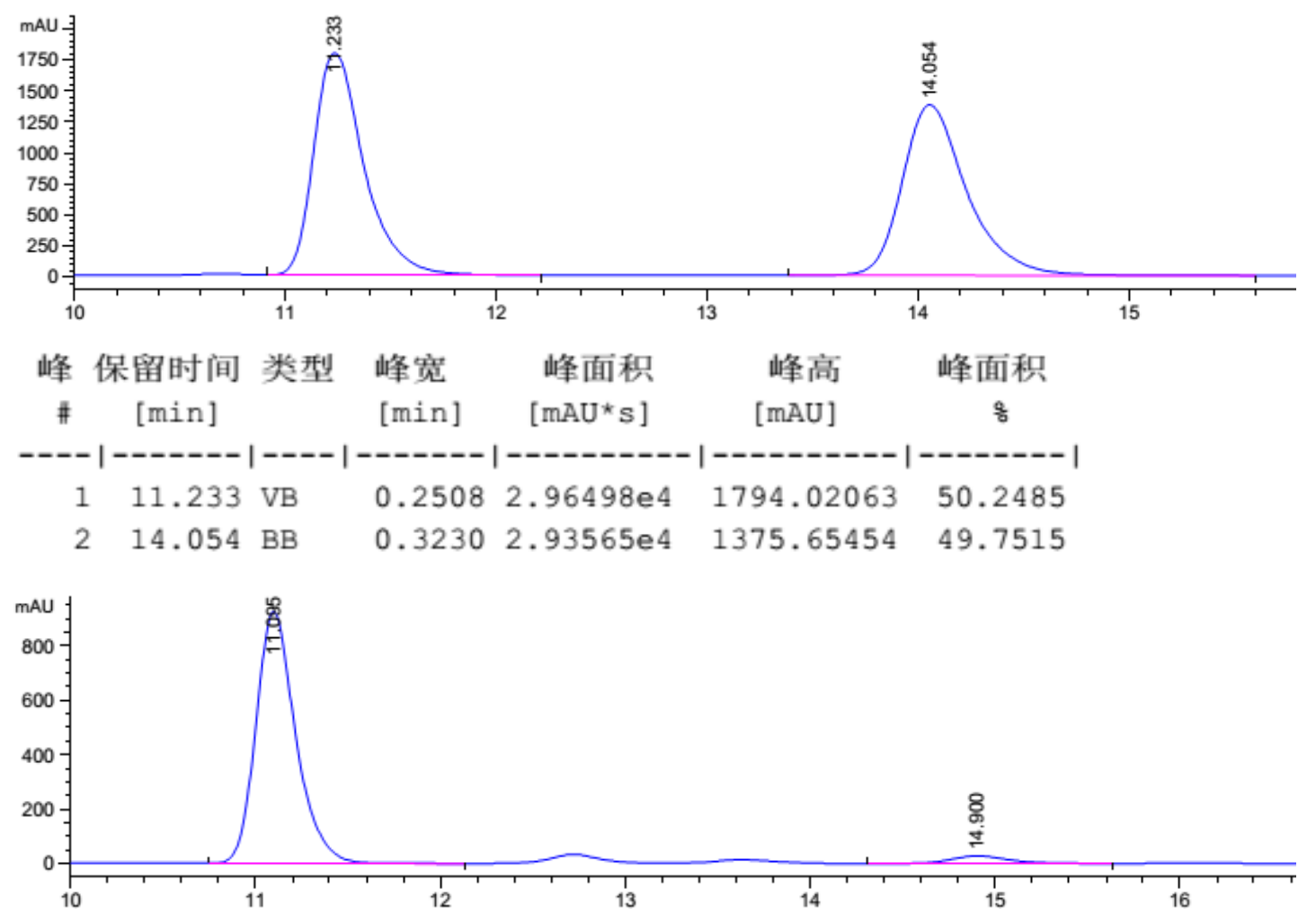


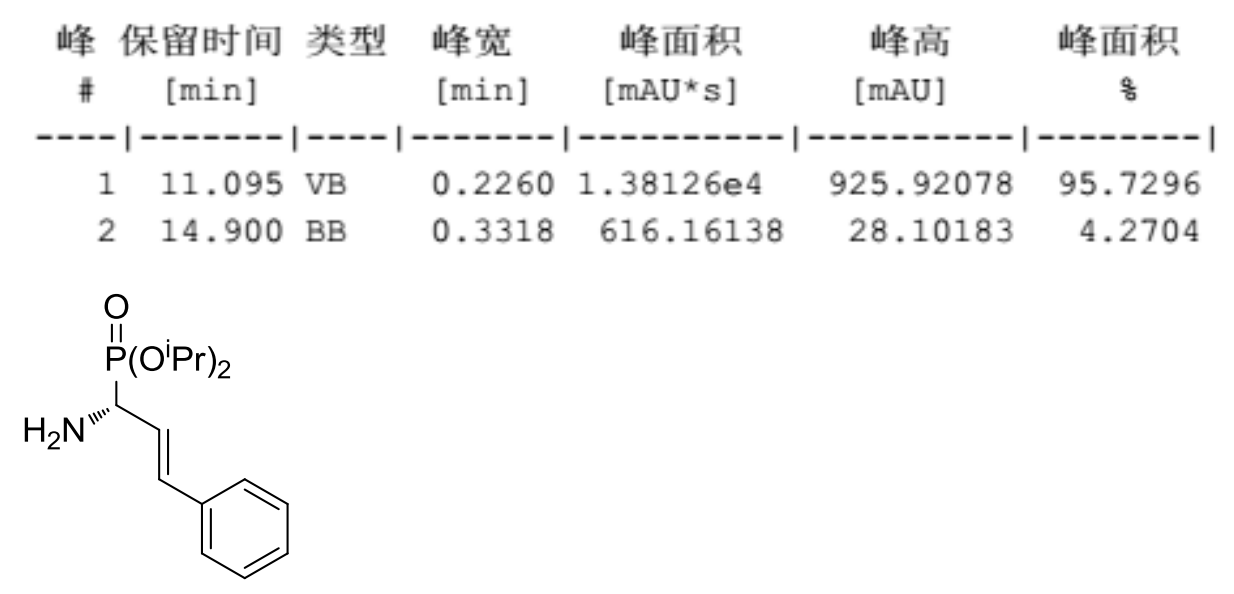

Cleavage of p-methoxyphenyl group of $\mathbf{4 b} .^{4}$<smiles>CCCOP(=O)(OCCC)C(/C=C/c1ccccc1)Nc1ccc(OC)cc1</smiles>

ee $64 \%$

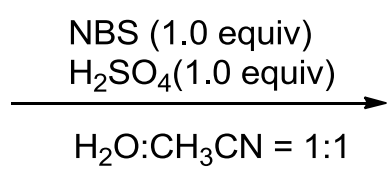

$\mathrm{H}_{2} \mathrm{O}: \mathrm{CH}_{3} \mathrm{CN}=1: 1$<smiles>CCCOP(=O)(OCC)[C@H](N)/C=C/c1ccccc1</smiles>

$48 \%$, ee $64 \%$

NBS (35.6 mg, $0.2 \mathrm{mmol})$ and $\mathrm{H}_{2} \mathrm{SO}_{4}(0.2 \mathrm{mmol}, 1 \mathrm{~mol} / \mathrm{L})$ were added to a solution of (R)-4b ( $80.6 \mathrm{mg}$, $0.2 \mathrm{mmol}$ ) in $\mathrm{H}_{2} \mathrm{O} / \mathrm{CH}_{3} \mathrm{CN}$ ( $\left.4.0 \mathrm{~mL}, 1: 1\right)$, and the whole mixture was stirred at room temperature. After completion of the reaction monitored by TLC, then $\mathrm{NaHCO}_{3}$ aq. was added, and aqueous layer was extracted with $\mathrm{Et}_{2} \mathrm{O}$ and dried over by $\mathrm{Na}_{2} \mathrm{SO}_{4}$. Solvent was removed under reduced pressure, the crude product was purified by silica gel column chromatography on silica gel using n-pentane/EtOAc (1:1) as the eluent to afford (R)-5 (28.5mg) in $48 \%$ yield..

\section{( $R, E)$-diisopropyl(1-amino-3-phenylallyl)phosphonate}

$\mathbf{5 : 4 8 \%}$ yield (28. $5 \mathrm{mg}$, n-pentane/EtOAc $=1: 1$ as the eluent). ${ }^{1} \mathbf{H} \mathbf{~ N M R ~}\left(\mathbf{4 0 0} \mathbf{~ M H z}, \mathbf{C D C l}_{\mathbf{3}}\right) \delta 7.56-$ $7.21(\mathrm{~m}, 5 \mathrm{H}), 6.77-6.53(\mathrm{~m}, 1 \mathrm{H}), 6.31(\mathrm{dt}, J=15.9,6.2 \mathrm{~Hz}, 1 \mathrm{H}), 4.86-4.59(\mathrm{~m}, 2 \mathrm{H}), 3.84(\mathrm{dt}, J=$ 29.8, $9.4 \mathrm{~Hz}, 1 \mathrm{H}), 2.32$ (brs, 1H), $1.50-1.14$ (m, 12H). $\left.{ }^{13} \mathbf{C} \mathbf{~ N M R ~ ( 1 0 1 ~} \mathbf{~ M H z}, \mathbf{C D C l}_{\mathbf{3}}\right) \delta 136.60,131.80$ $(\mathrm{d}, J=13.0 \mathrm{~Hz}), 128.55,127.67,126.44(\mathrm{~d}, J=1.8 \mathrm{~Hz}), 125.57(\mathrm{~d}, J=6.1 \mathrm{~Hz}), 71.12(\mathrm{~d}, J=7.4 \mathrm{~Hz})$, $52.48(\mathrm{~d}, J=150.7 \mathrm{~Hz}), 24.12(\mathrm{~d}, J=3.5 \mathrm{~Hz}), 23.99(\mathrm{~d}, J=5.1 \mathrm{~Hz}) .{ }^{31} \mathbf{P}$ NMR $\left(\mathbf{1 6 2} \mathbf{~ M H z}, \mathbf{C D C l}_{3}\right) \delta$ 23.00. Enantiomeric excess is $64 \%$ determined by HPLC analysis: Chiralcel AD-H (hexane/iPrOH = $80 / 20$, flow rate $=1.0 \mathrm{~mL} / \mathrm{min}, 240.4 \mathrm{~nm}), \mathrm{t}_{\mathrm{r}}$ (major) $=5.125 \mathrm{~min}, \mathrm{t}_{\mathrm{r}}($ minor $)=6.267 \mathrm{~min} .[\alpha]_{\mathrm{D}}^{22.7}+14$ $\left(\mathrm{c}=1.0, \mathrm{CHCl}_{3}\right)\left(64 \%\right.$ ee). HRMS (ESI) for $\mathrm{C}_{15} \mathrm{H}_{24} \mathrm{NO}_{3} \mathrm{PNa}\left([\mathrm{M}+\mathrm{Na}]^{+}\right)$: calcd 320.1386, found 320.1392 .

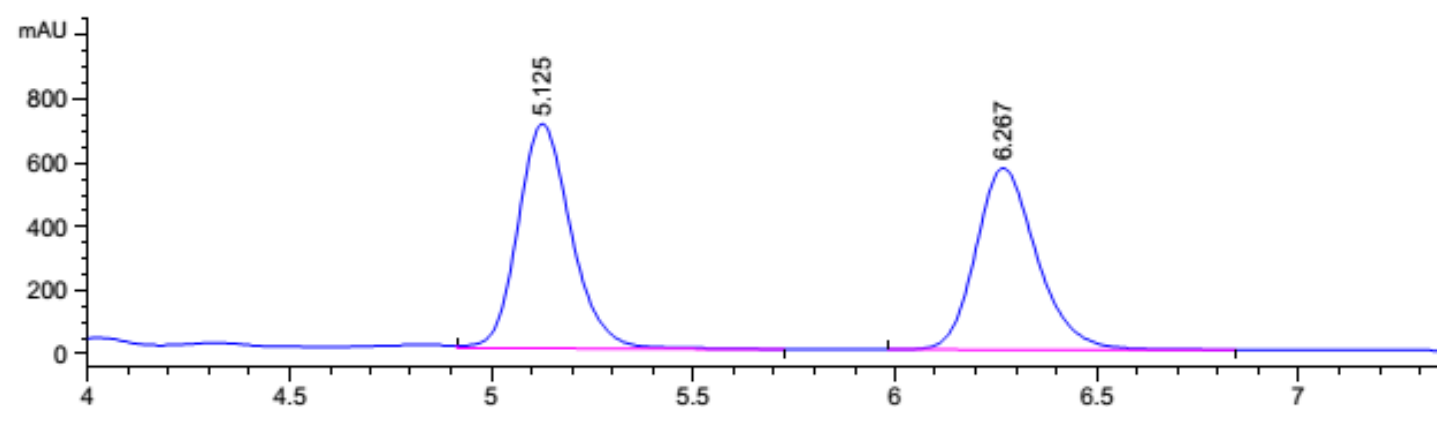



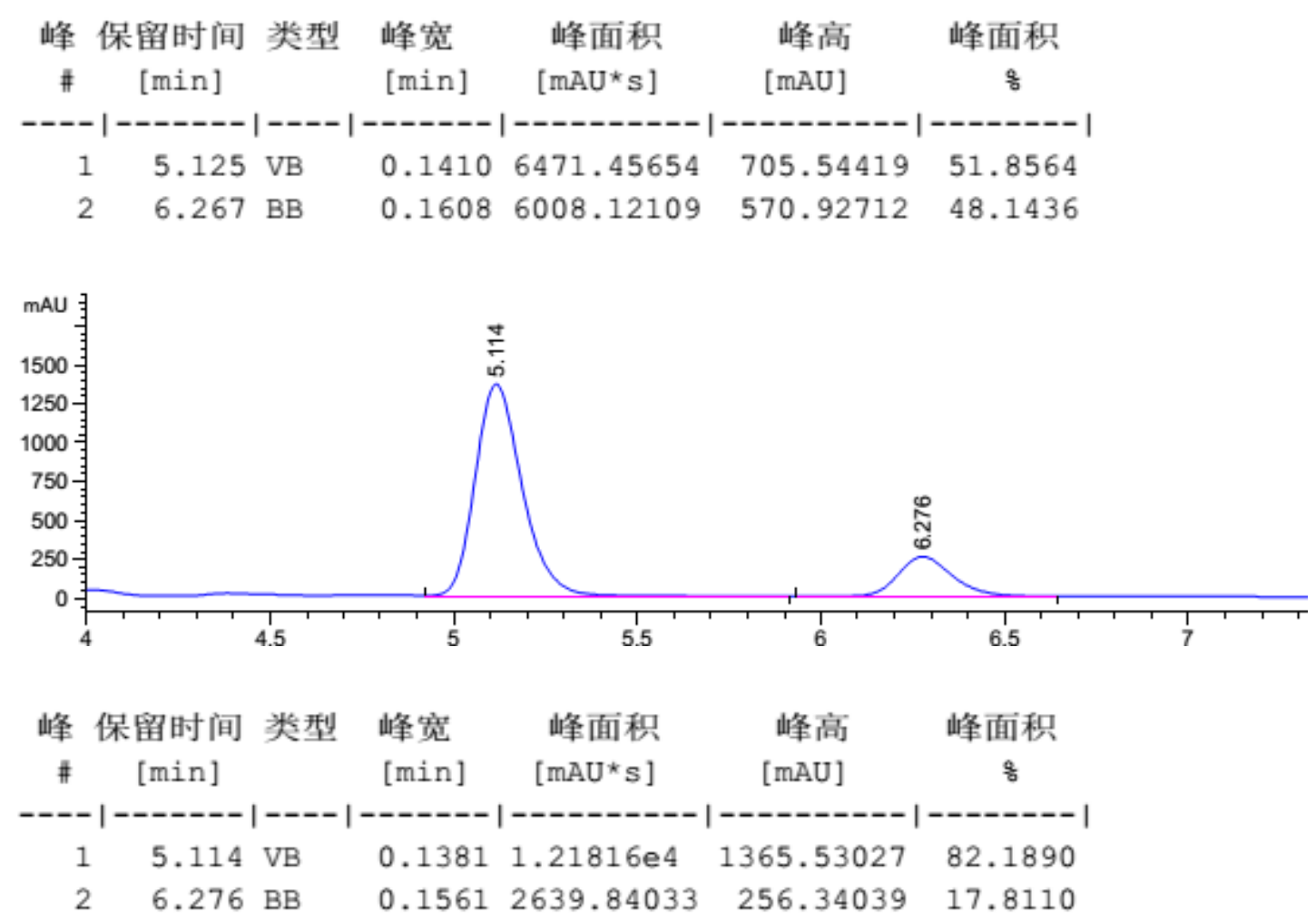

\section{Reference}

1. (a) Wipf. P.; Jung. J-K. J. Org. Chem. 2000, 65, 6319. (b) Momiyama, N.; Nishimoto, H.; Terada, M. Org. Lett. 2011, 13, 2126. (c) Neel, A. J.; Hehn, J. P.; Toste, F. D. J. Am. Chem. Soc. 2013, 135, 14044.

2. West, T. H.; Daniels, D. S. B.; Slawin, A. M. Z.; Smith, A. D. J. Am. Chem. Soc., 2014, 136, 4476.

3. Akiyama, T.; Morita, H.; Itoh, J.; Fuchibe, K. Org. Lett. 2005, 7, 2583.

4. Verkade, J. M. M.; van Hemert, L. J. C.; Quaedflieg, P. J. L. M.; Alsters, P. L.; van Delft, F. L.; Rutjes, F. P. J. T. Tetrahedron Lett. 2006, 47, 8109. 
VII. NMR Charts
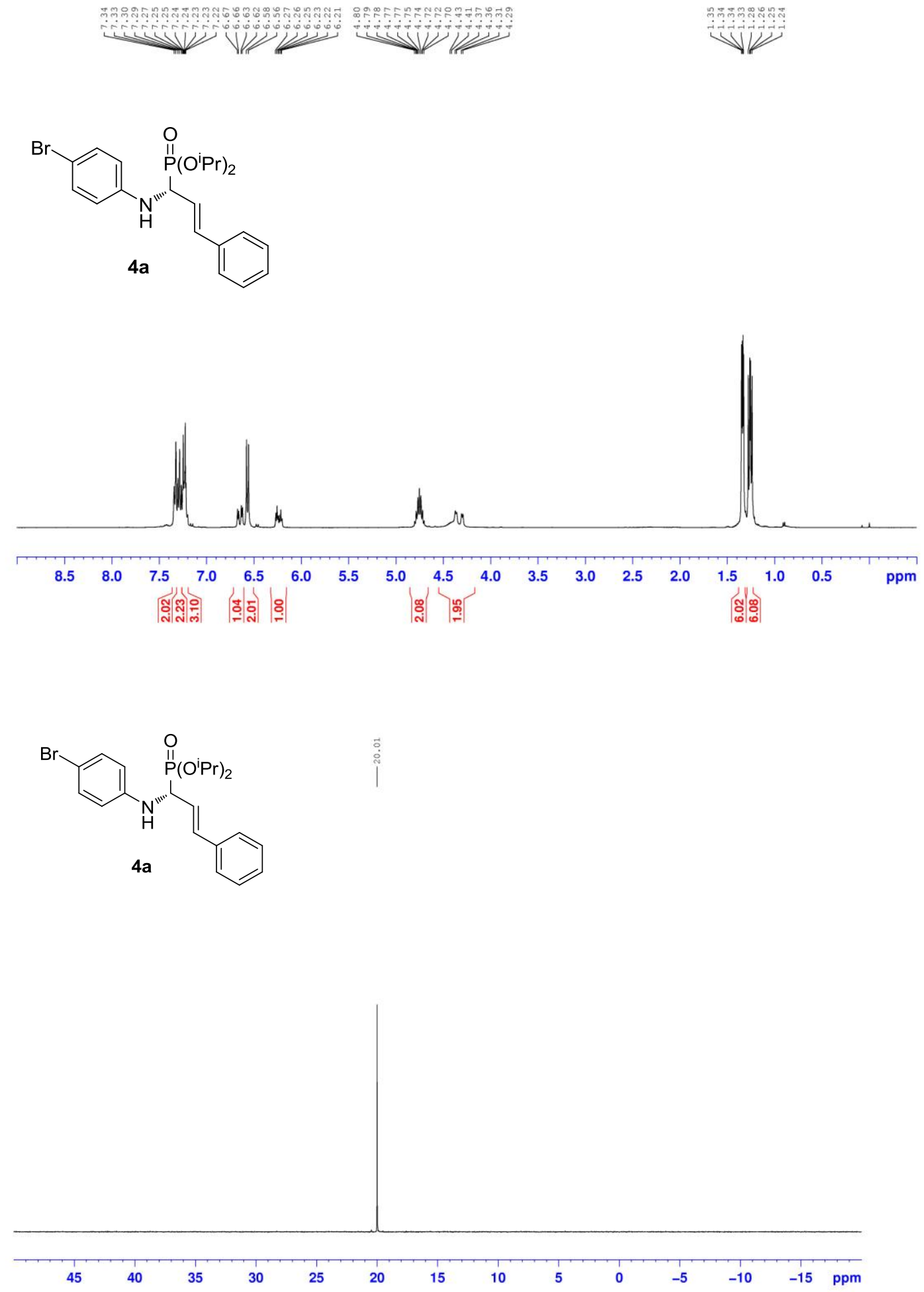

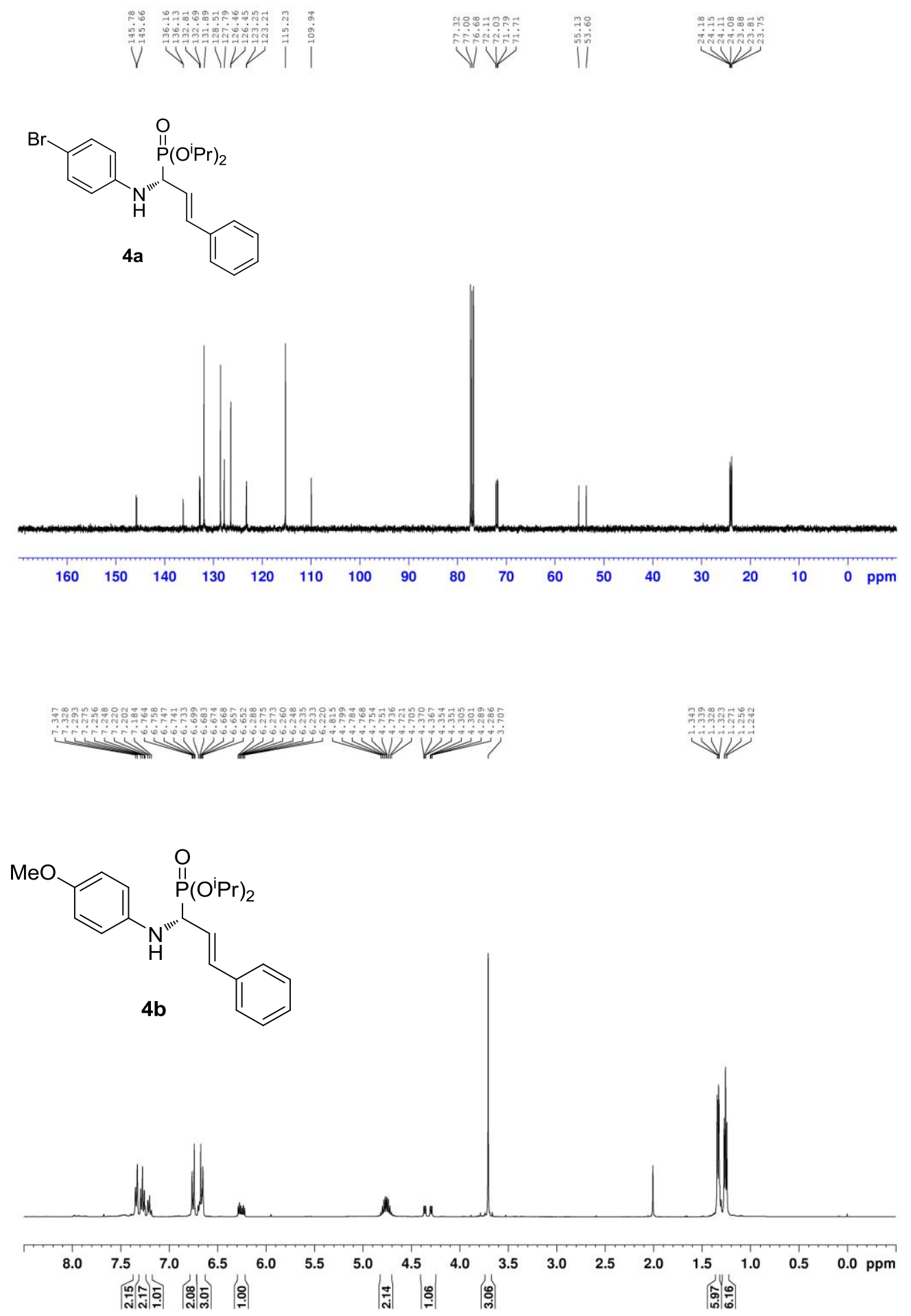

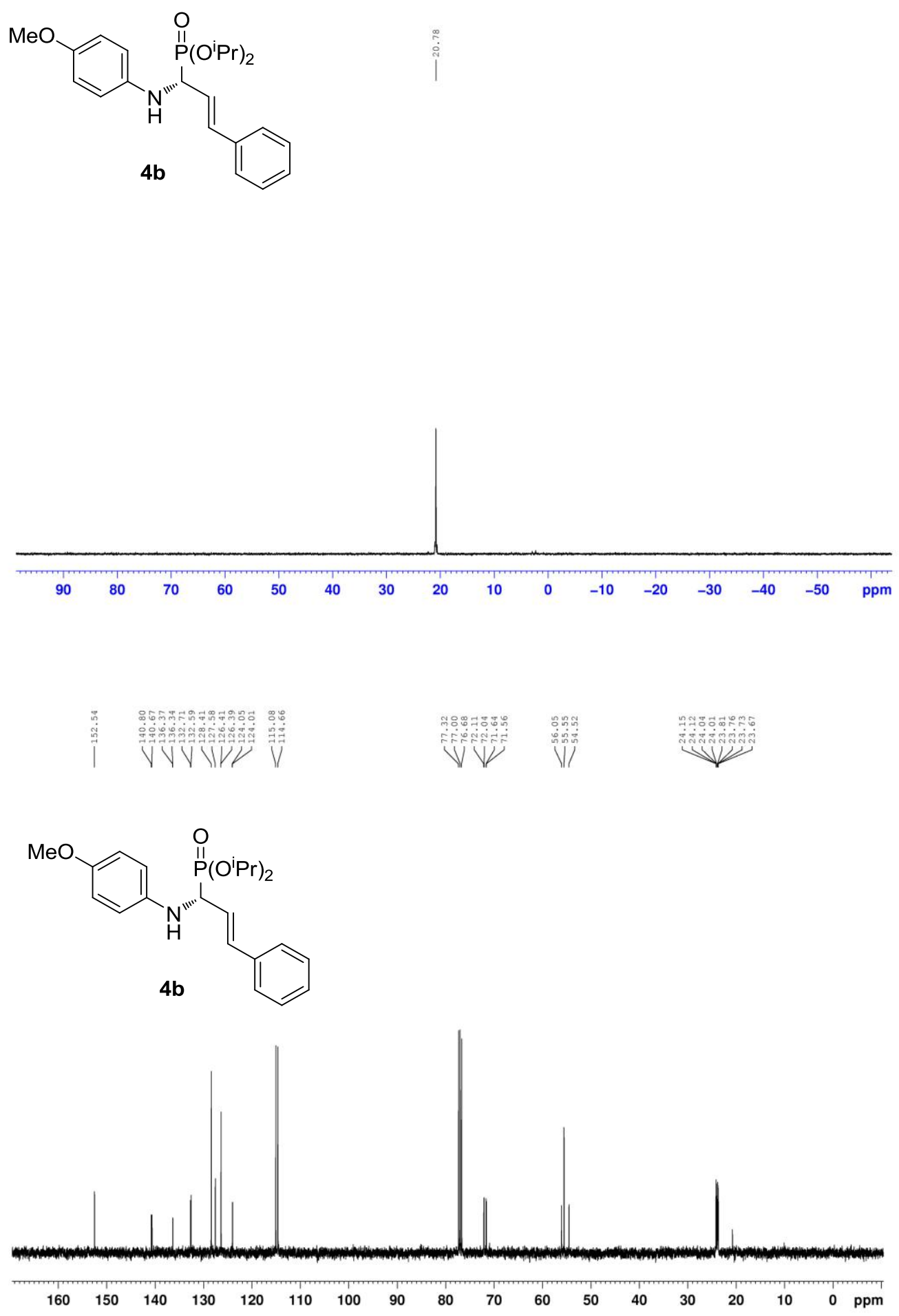

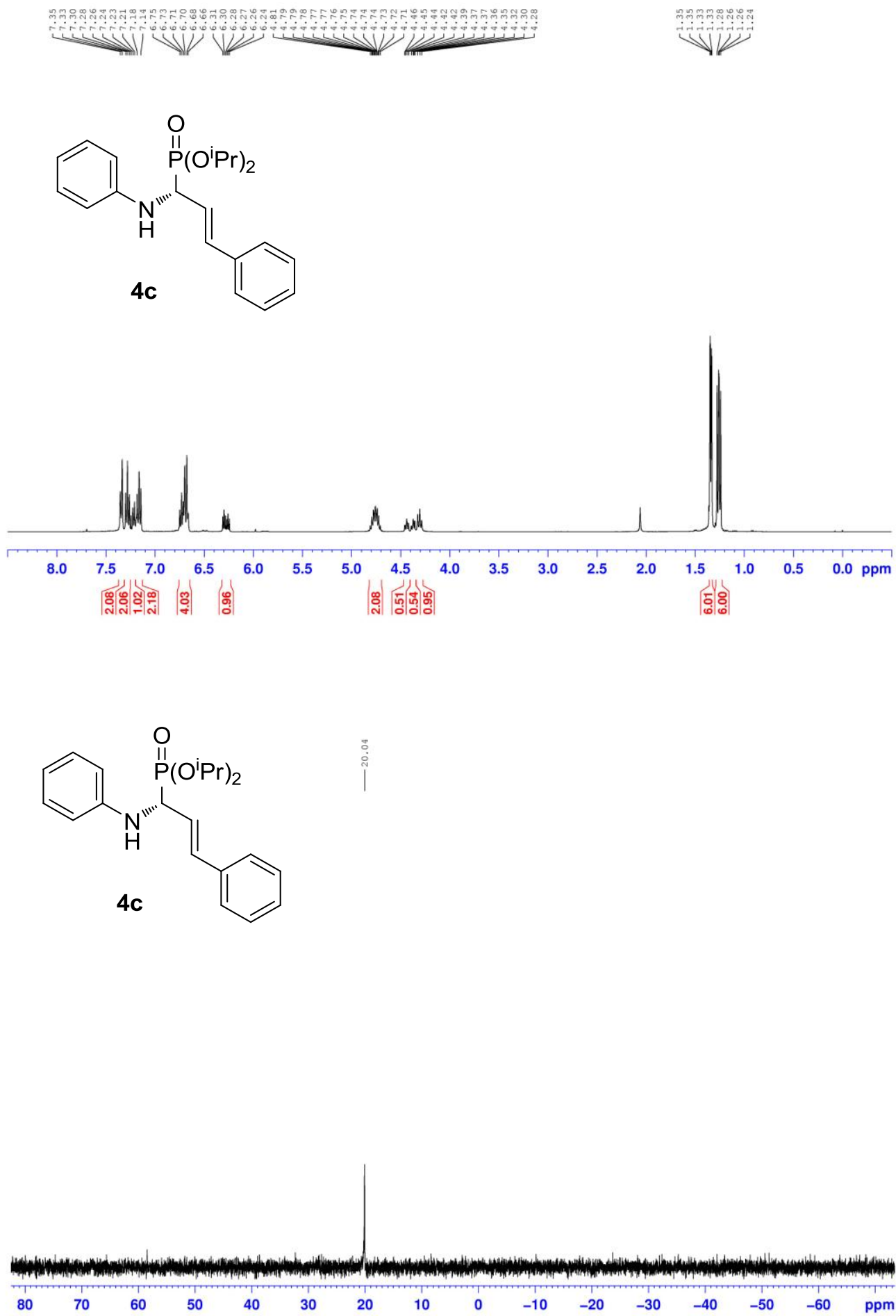

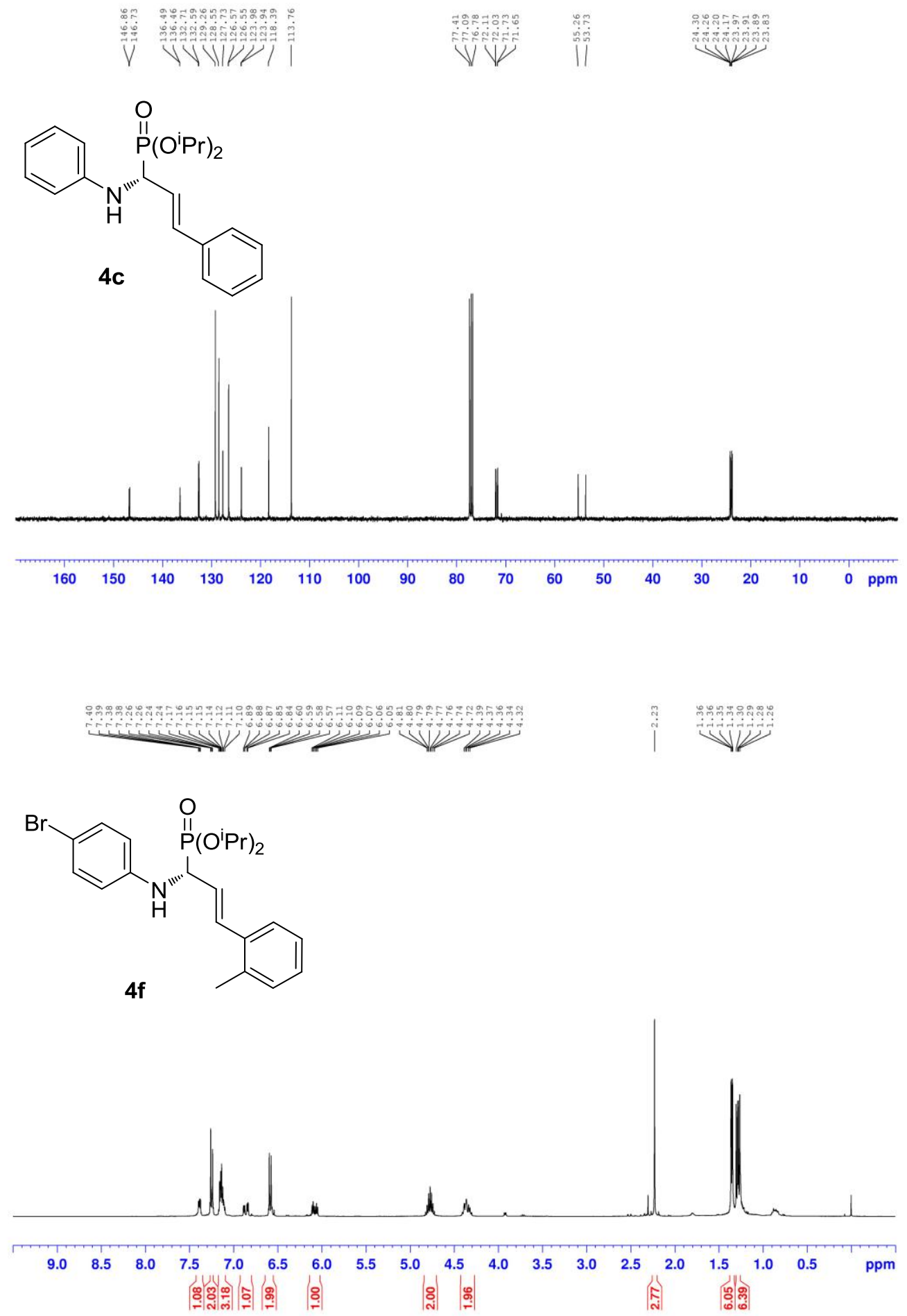

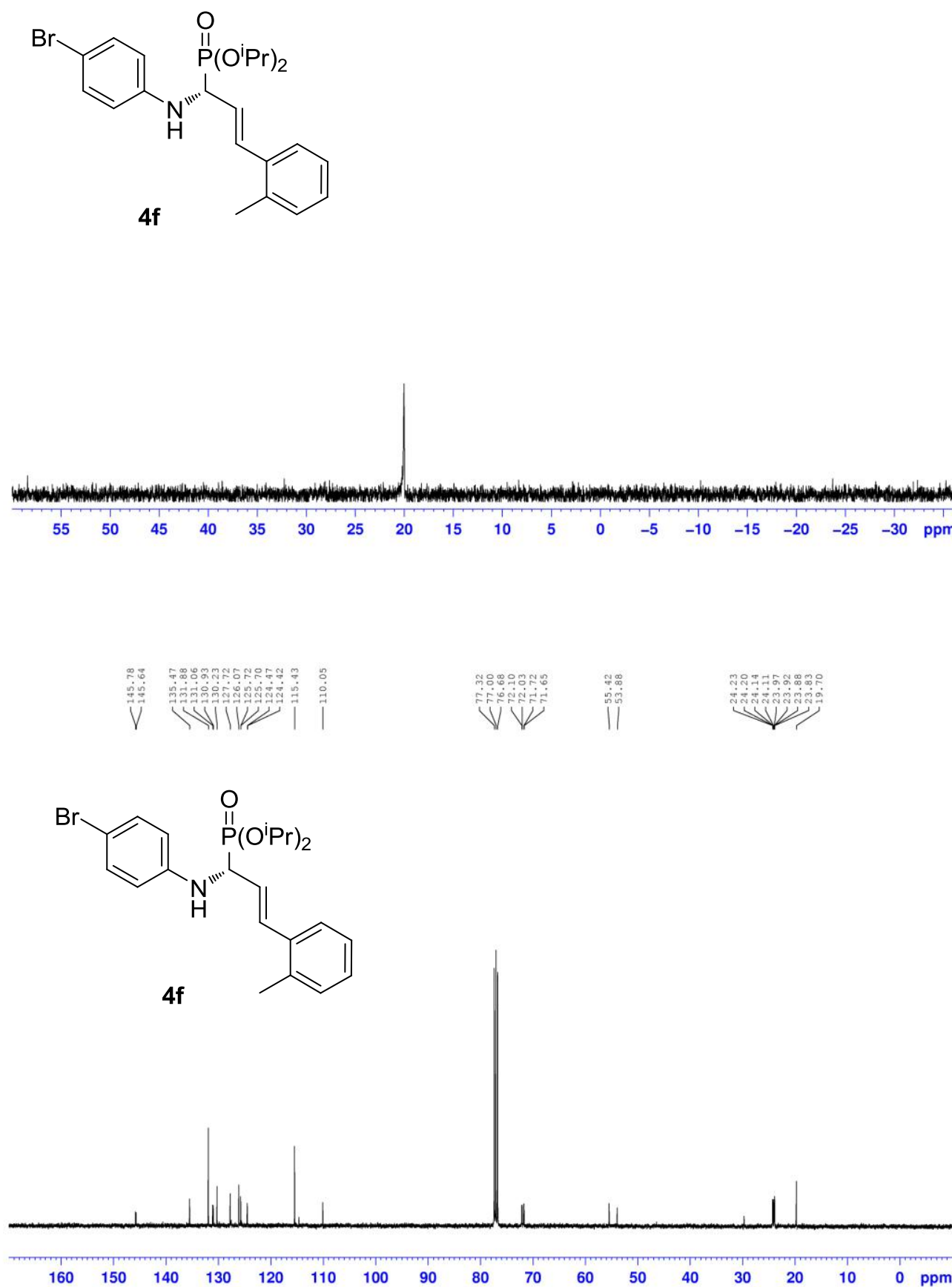
<smiles>O=[P+](O)C(/C=C/c1ccccc1F)Nc1ccc(Br)cc1</smiles>

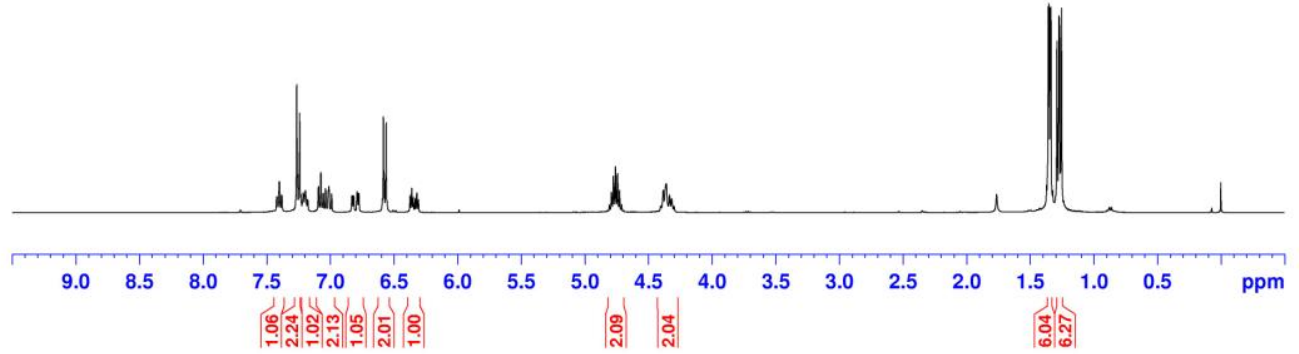<smiles>O=[PH](O)C(/C=C/c1ccccc1F)Nc1ccc(Br)cc1</smiles>

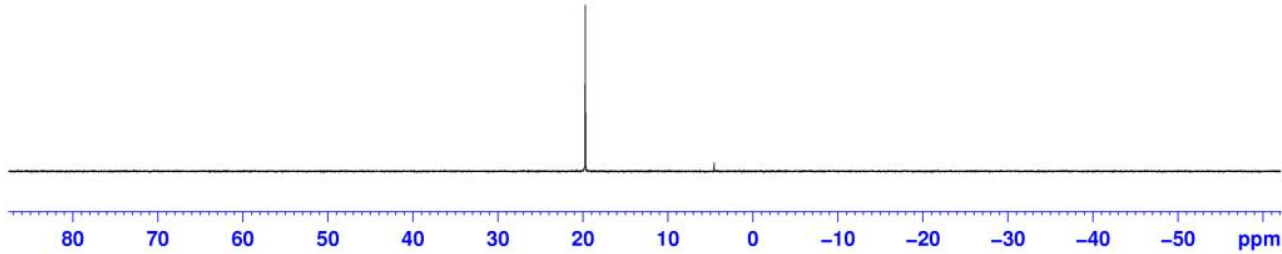


<smiles></smiles>
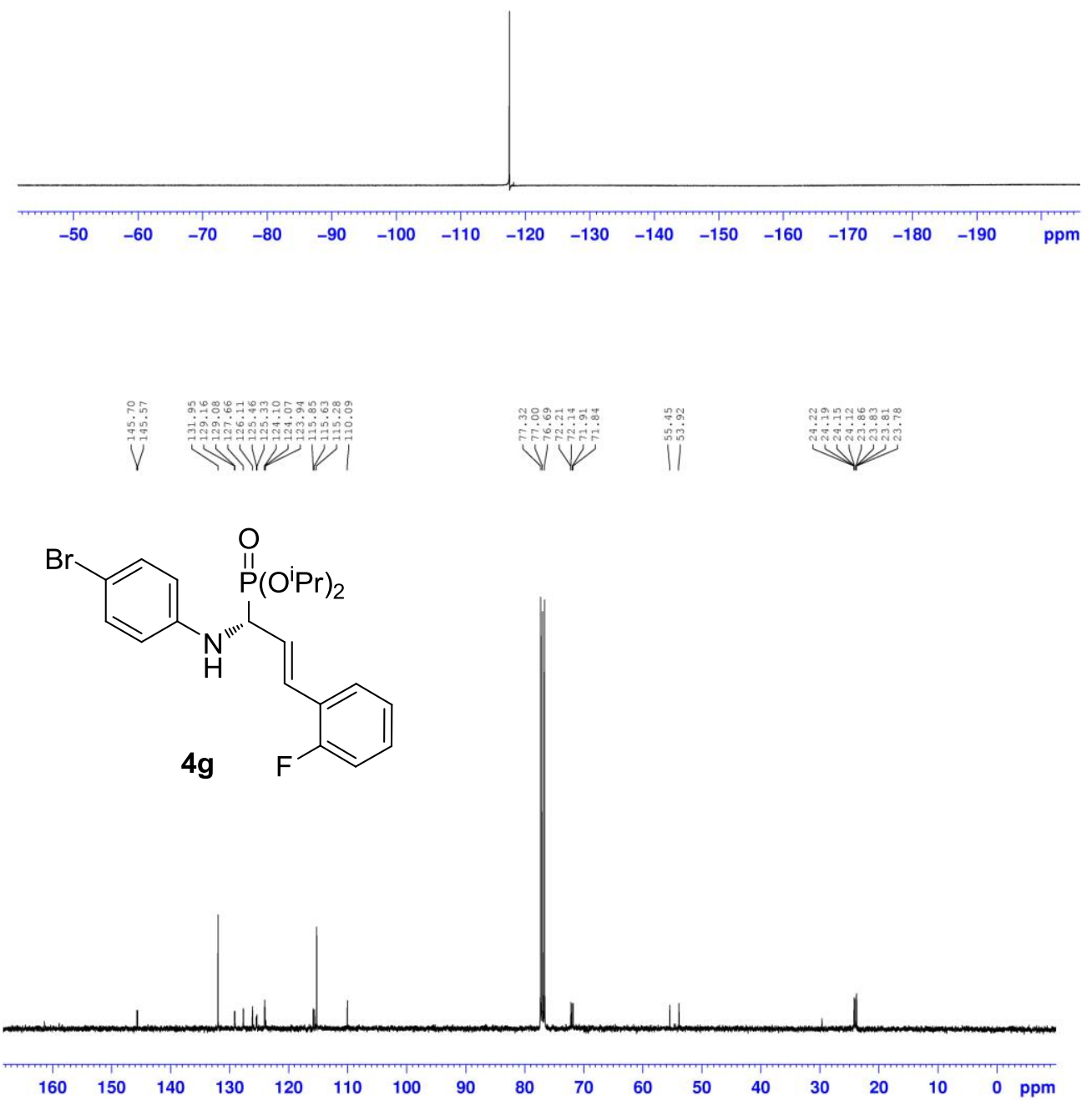

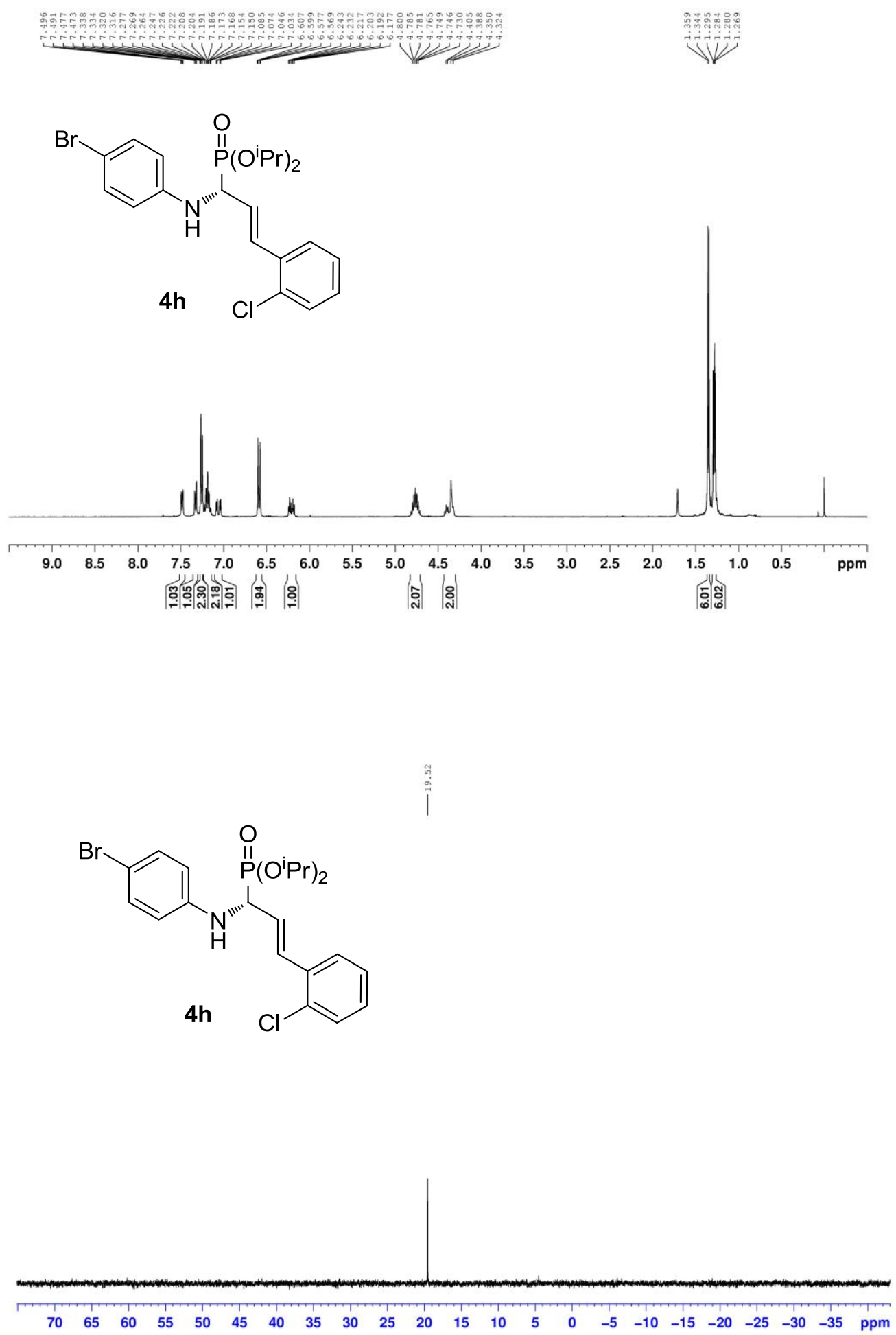


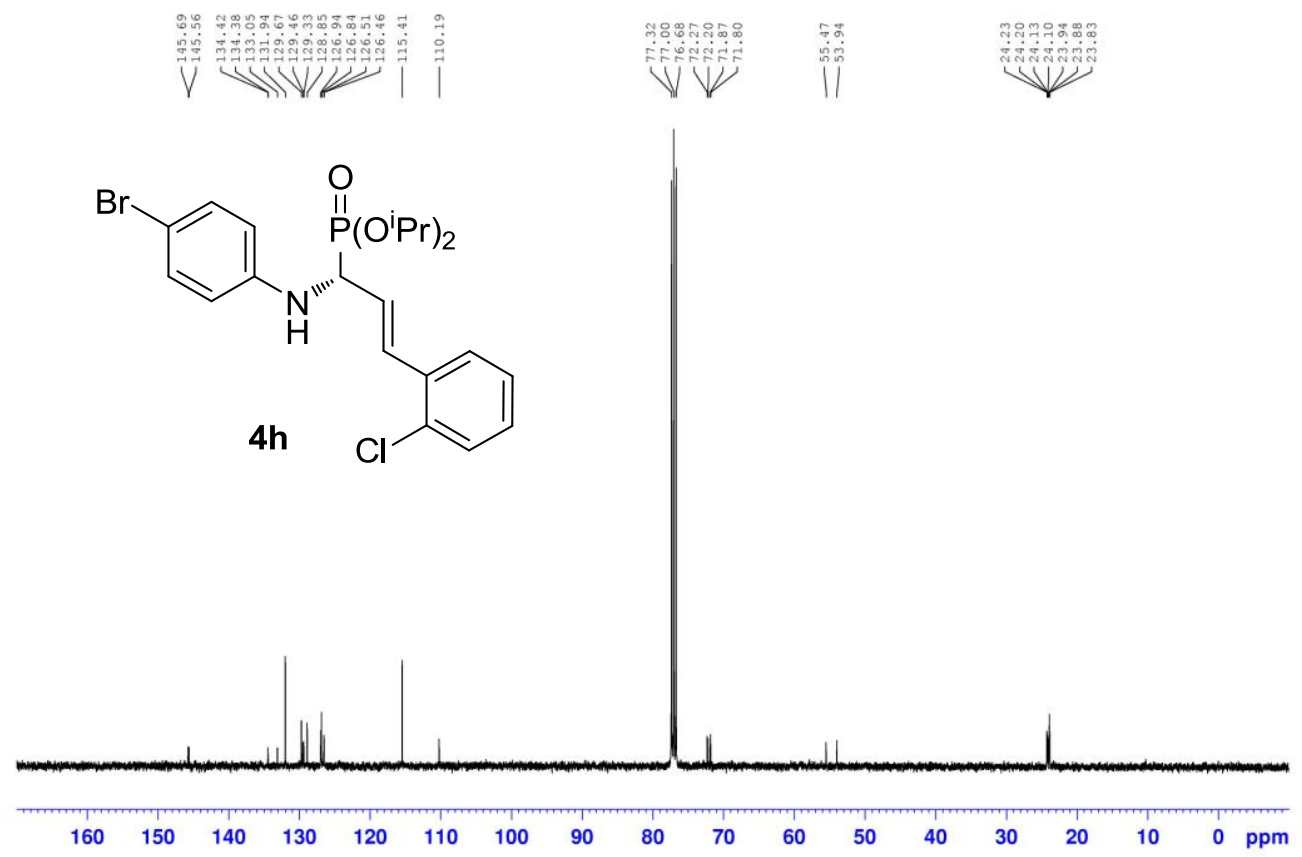

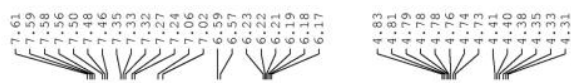
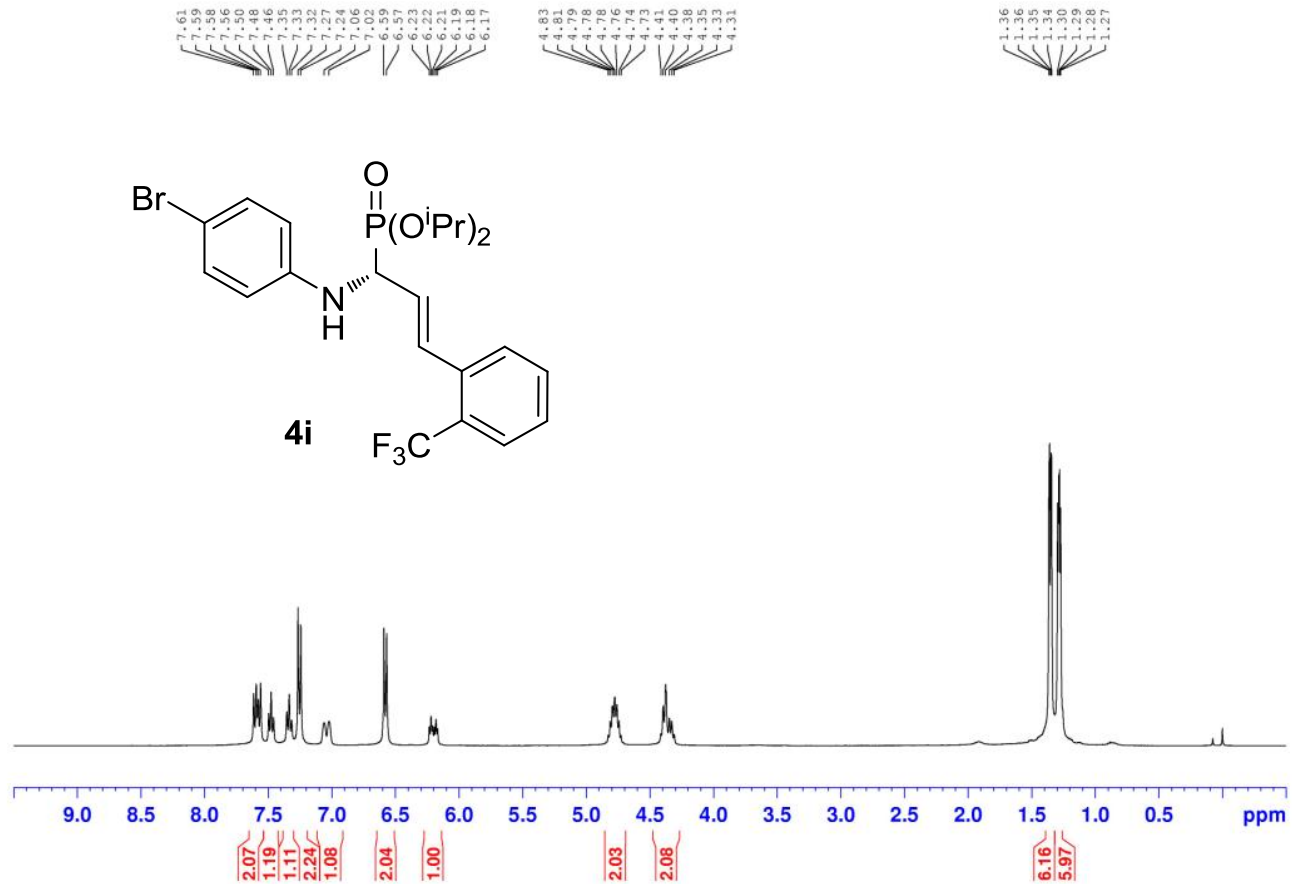

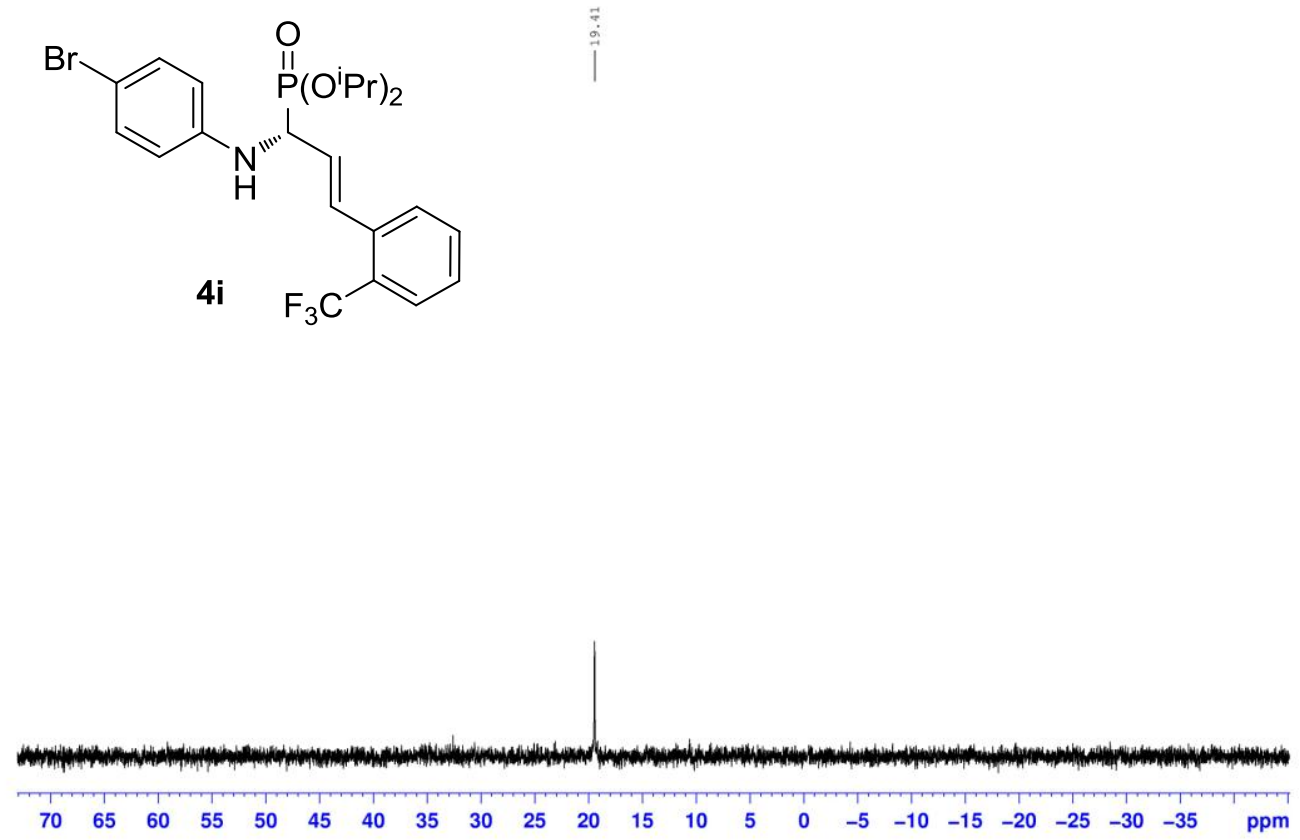<smiles>O=[PH](OCc1ccccc1)[C@H](/C=C/c1ccccc1C(F)(F)F)Nc1ccc(Br)cc1</smiles>

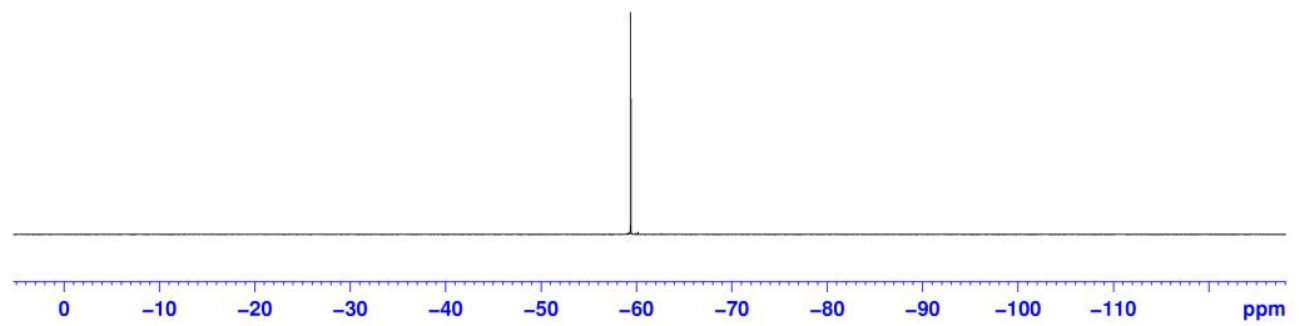



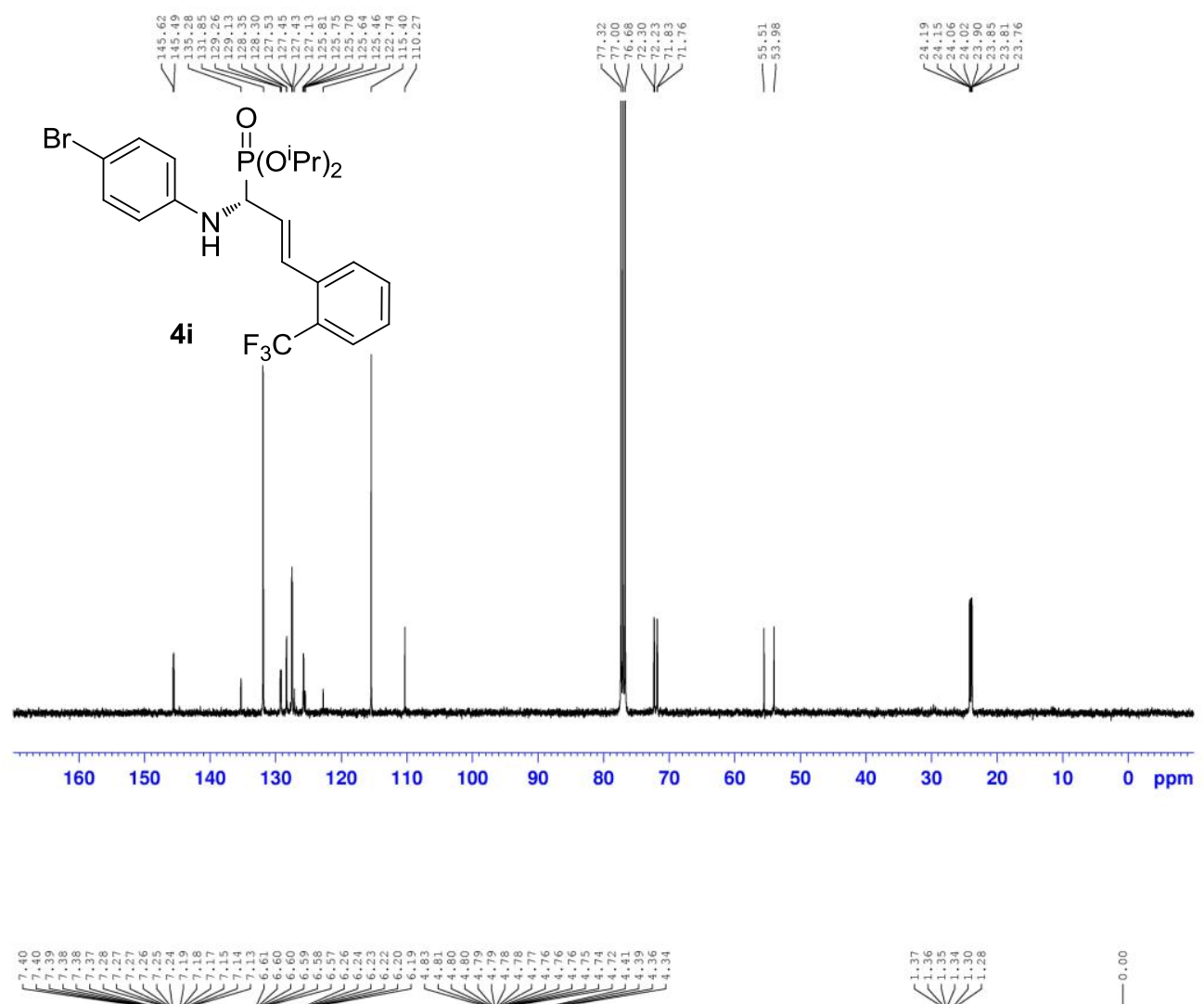<smiles>O=[N+]([O-])c1ccccc1/C=C/[C@H](Nc1ccc(Br)cc1)P(=O)(O)O</smiles>

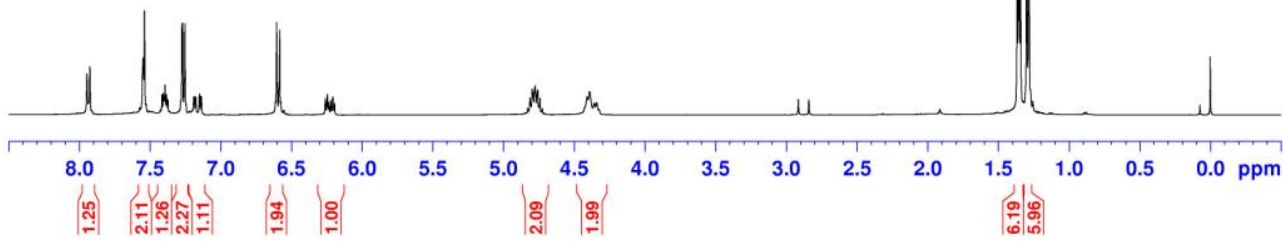



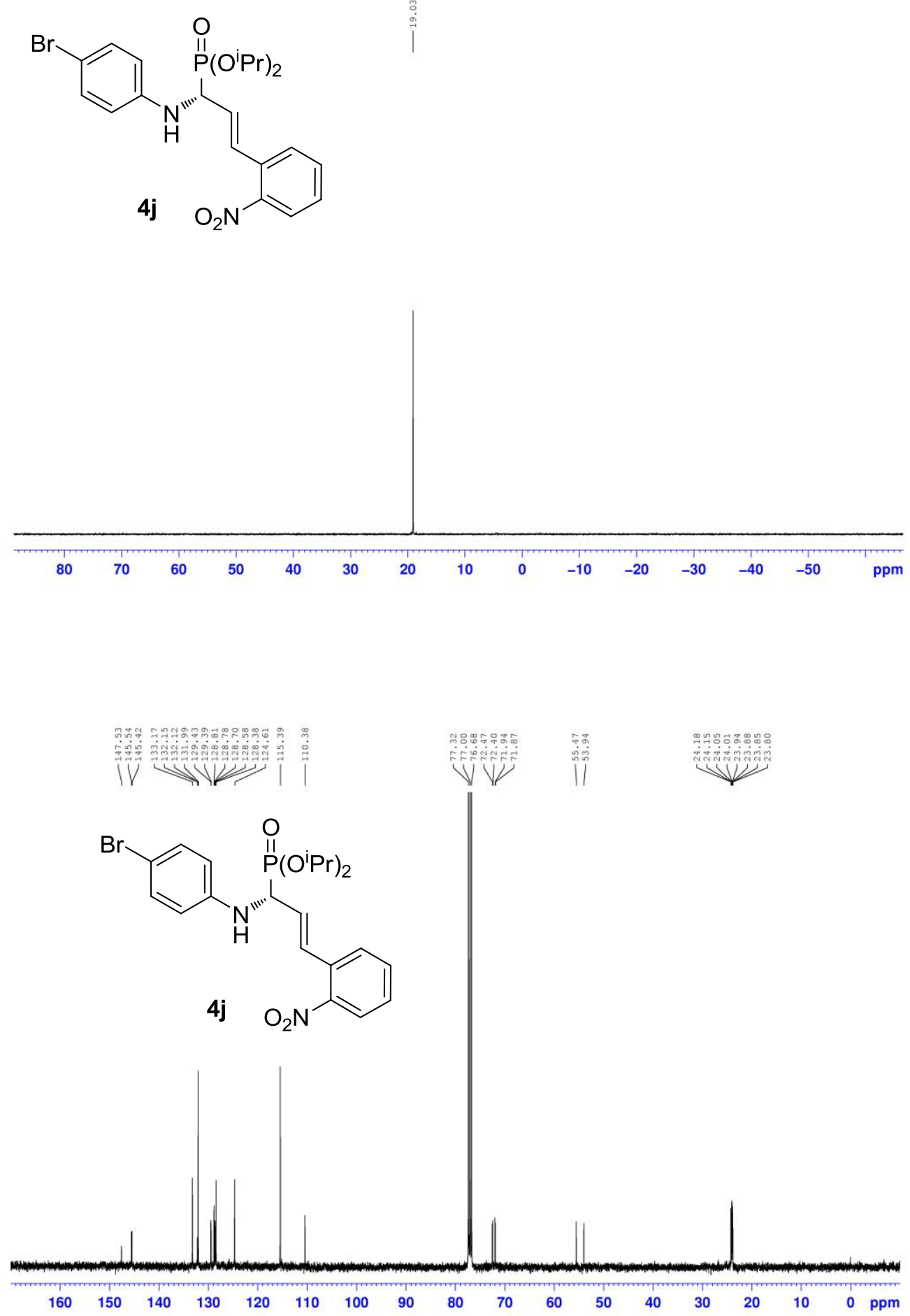

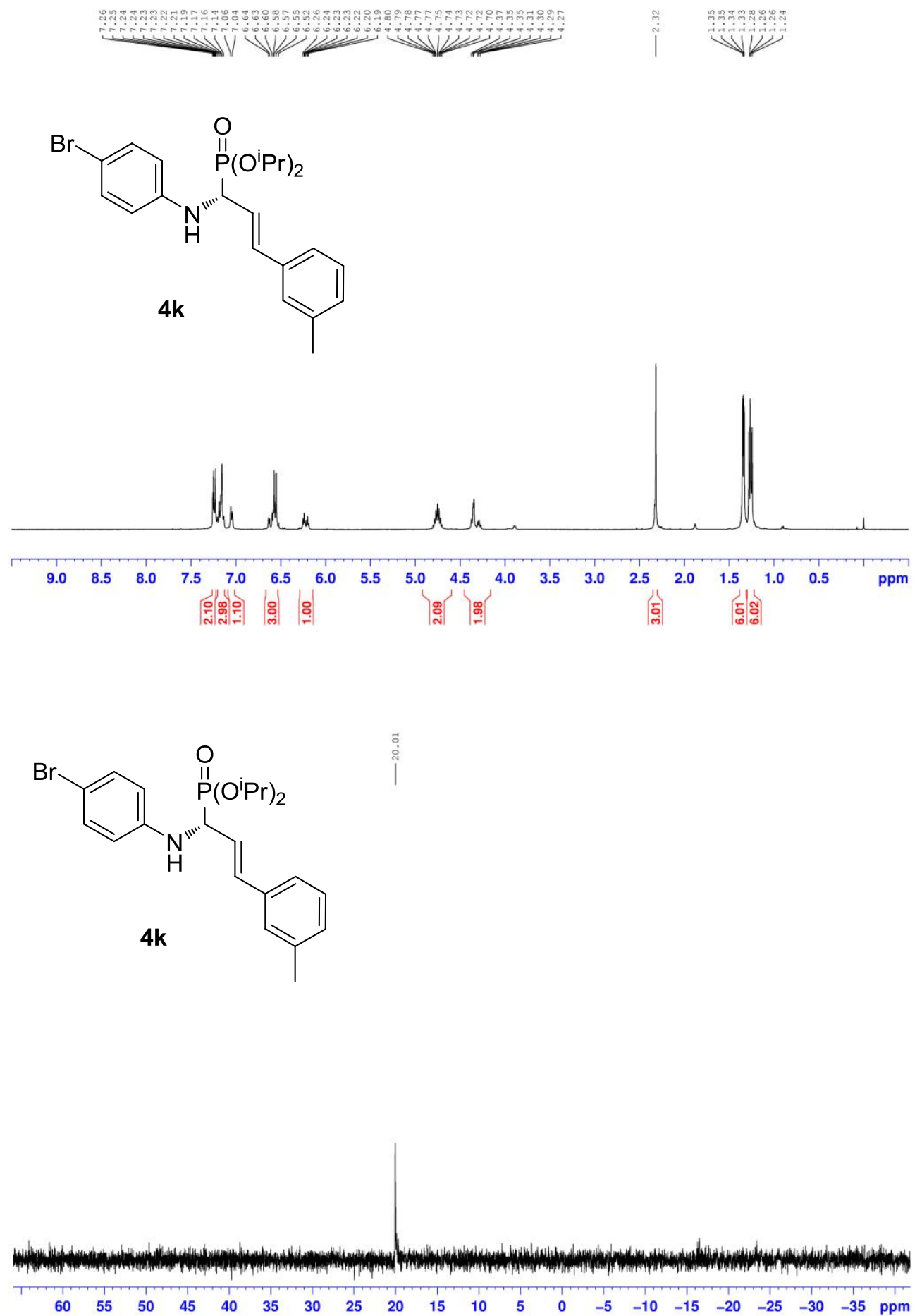

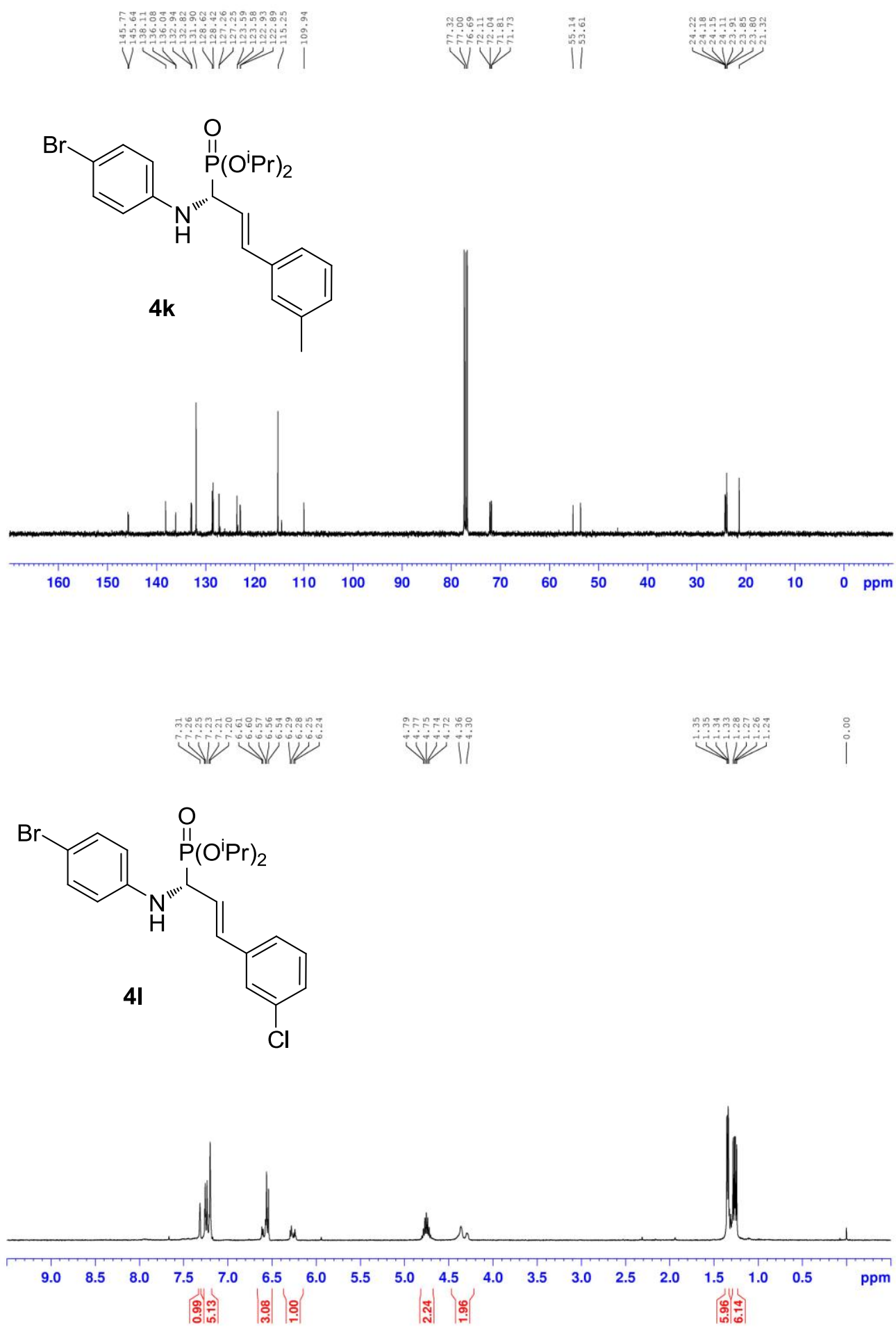
<smiles>O=[PH](OCc1ccccc1)[PH](=O)C(/C=C/c1cccc(Cl)c1)Nc1ccc(Br)cc1</smiles>
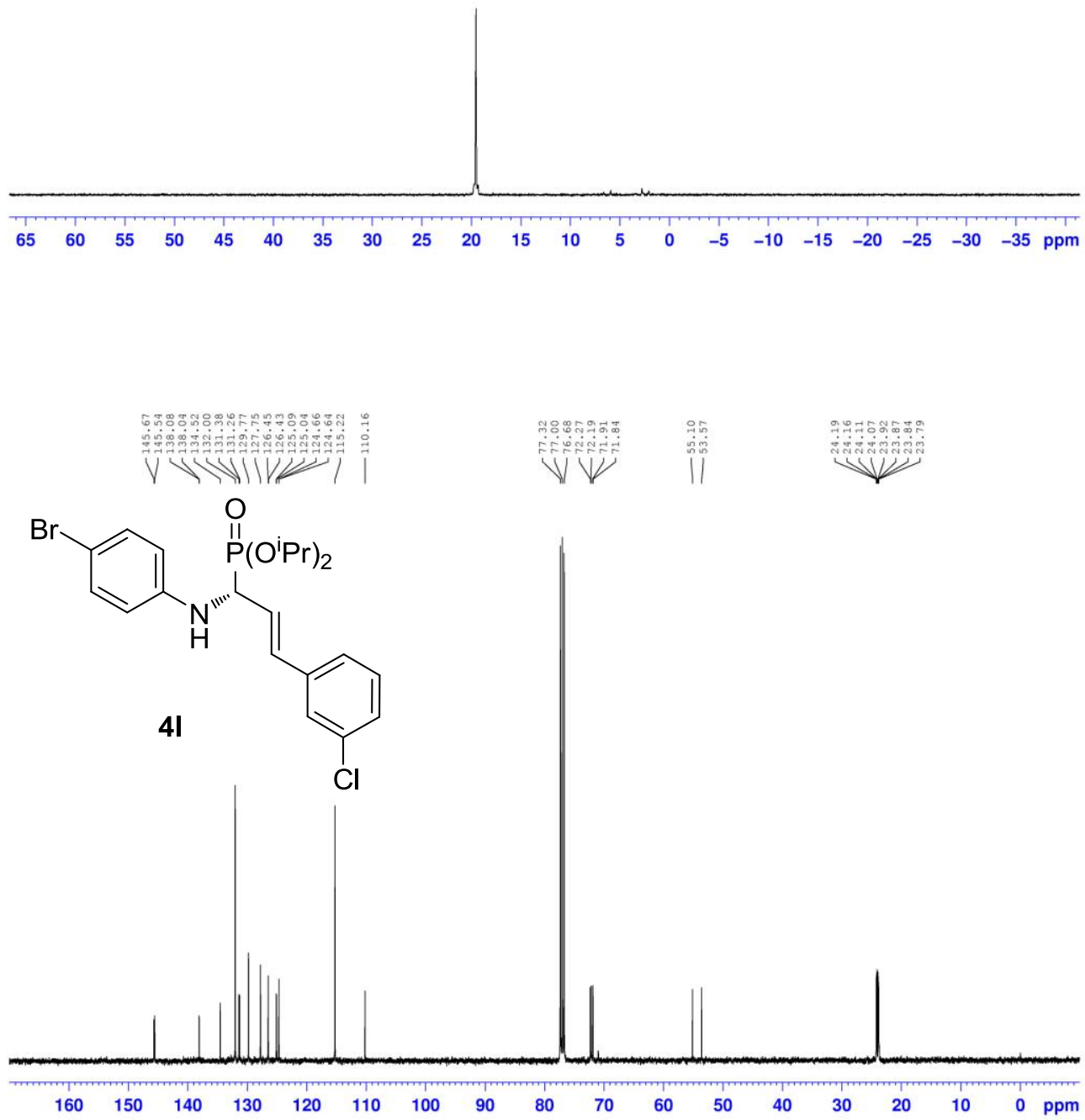
<smiles>Cc1ccc(/C=C/[C@H](Nc2ccc(Br)cc2)P(=O)(O)O)cc1</smiles>

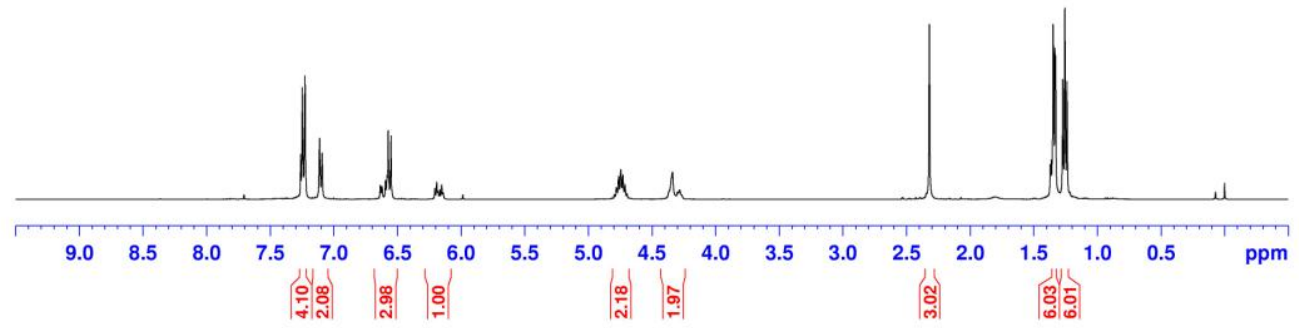<smiles>Cc1ccc(/C=C/[C@H](Nc2ccc(Br)cc2)P(=O)(O)O)cc1</smiles>

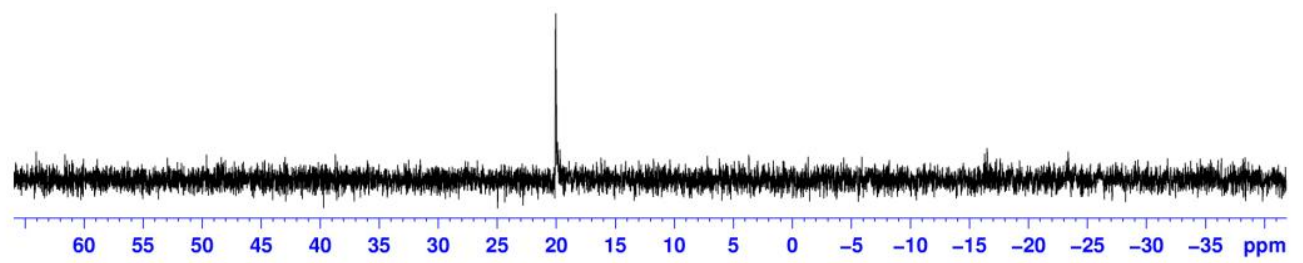




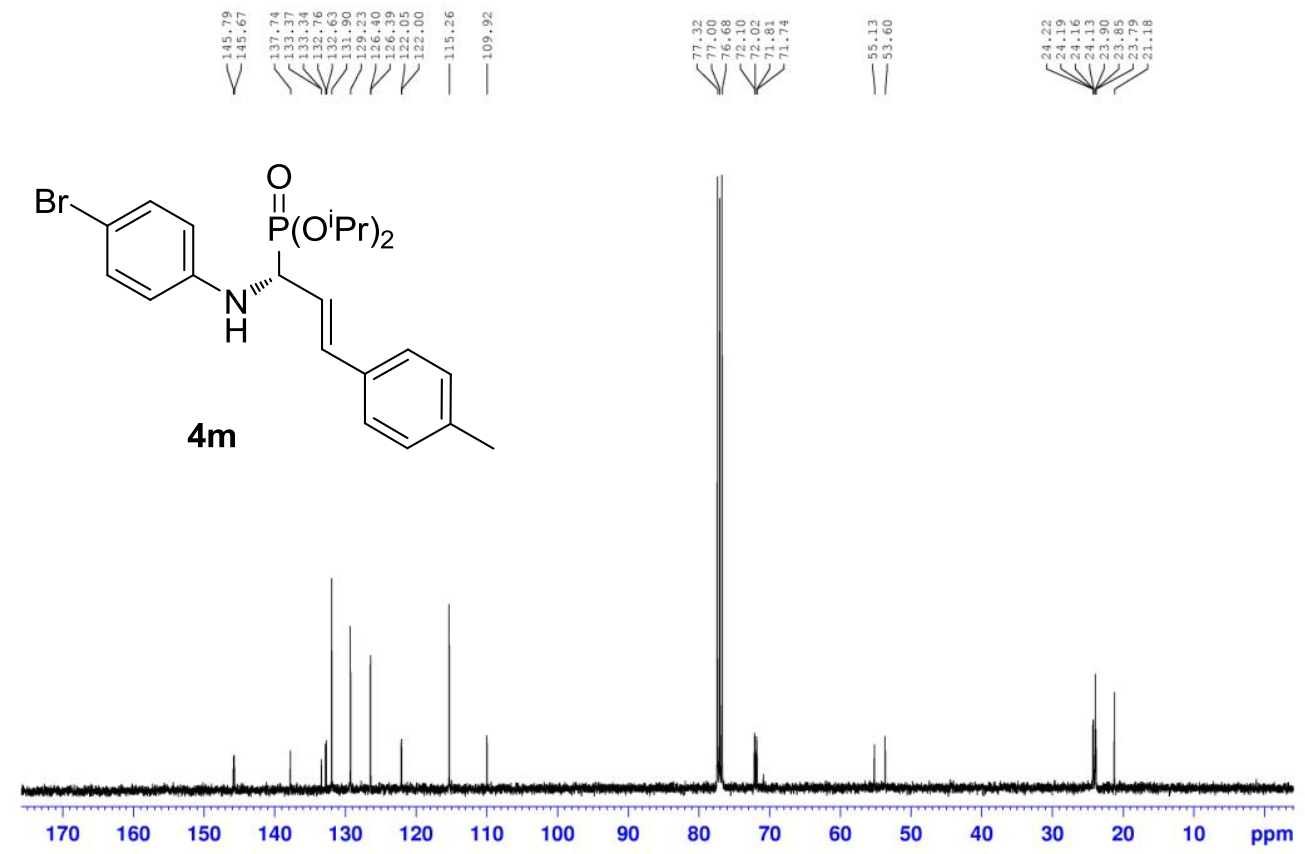

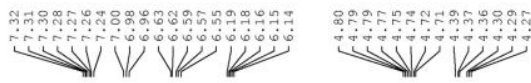
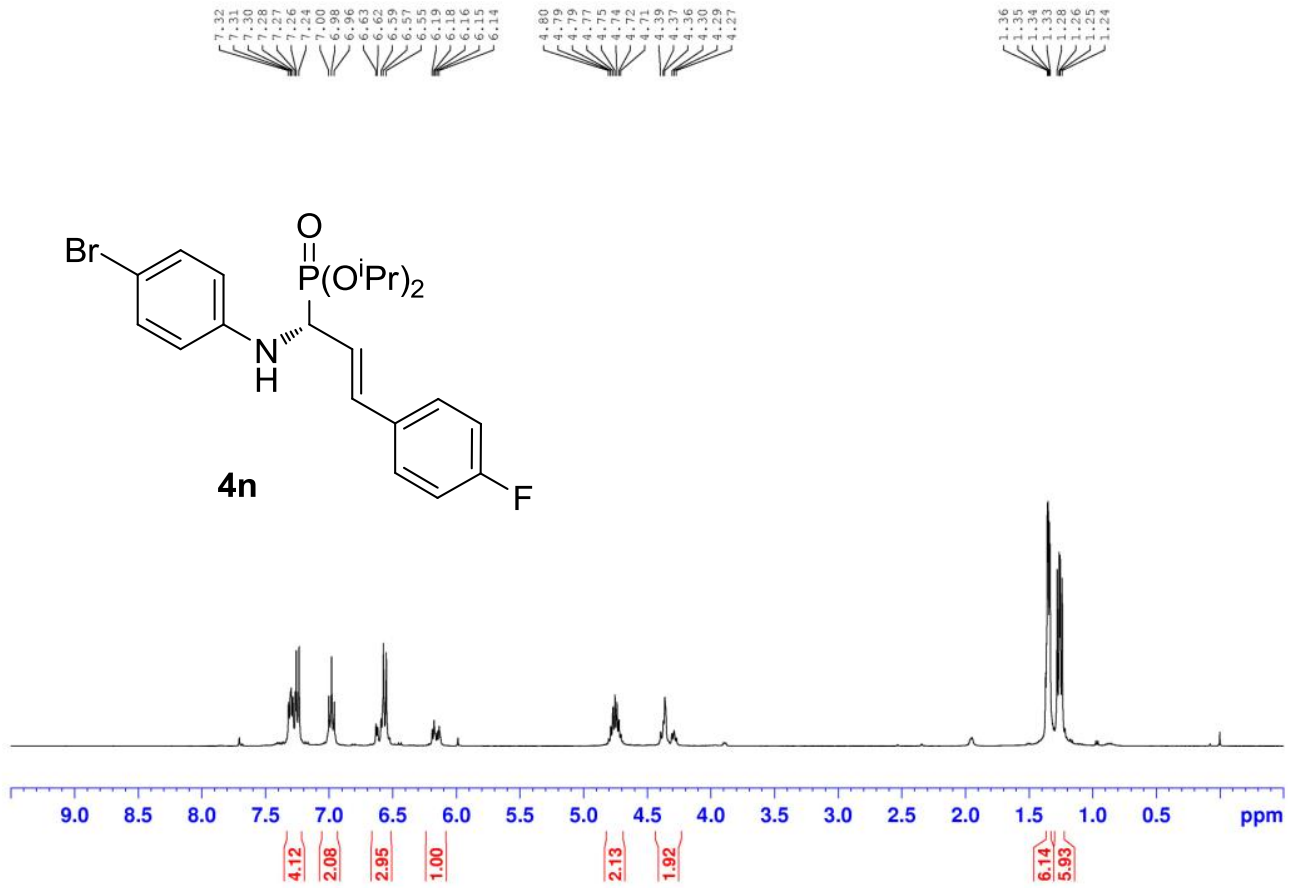
<smiles>O=[PH](OCc1ccccc1)[C@H](/C=C/c1ccc(F)cc1)Nc1ccc(Br)cc1</smiles>

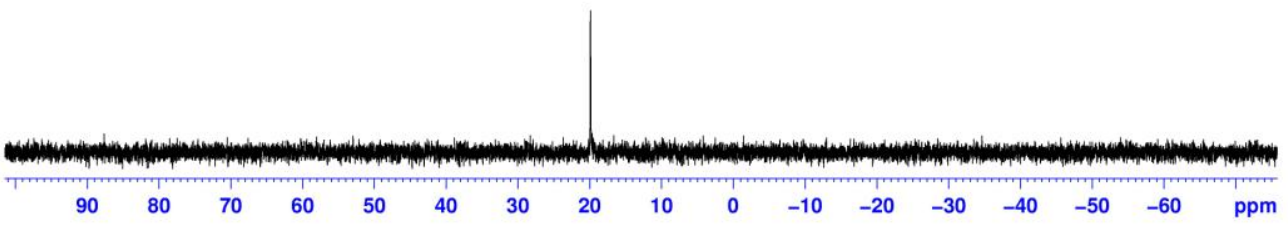<smiles>O=P[PH2+]C(/C=C/c1ccc(F)cc1)Nc1ccc(Br)cc1</smiles>

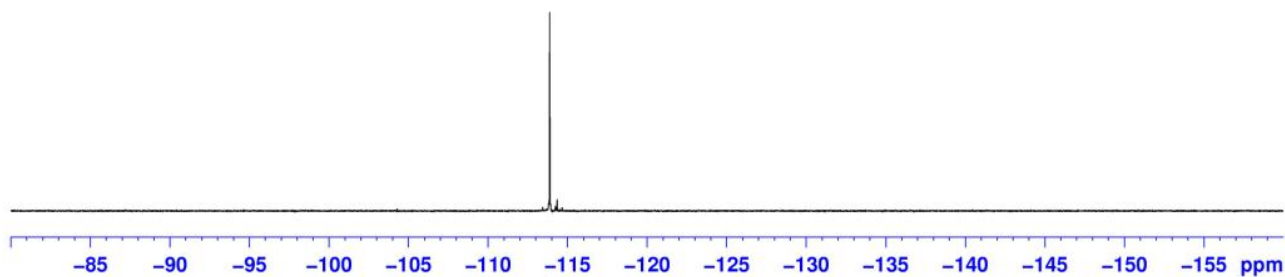



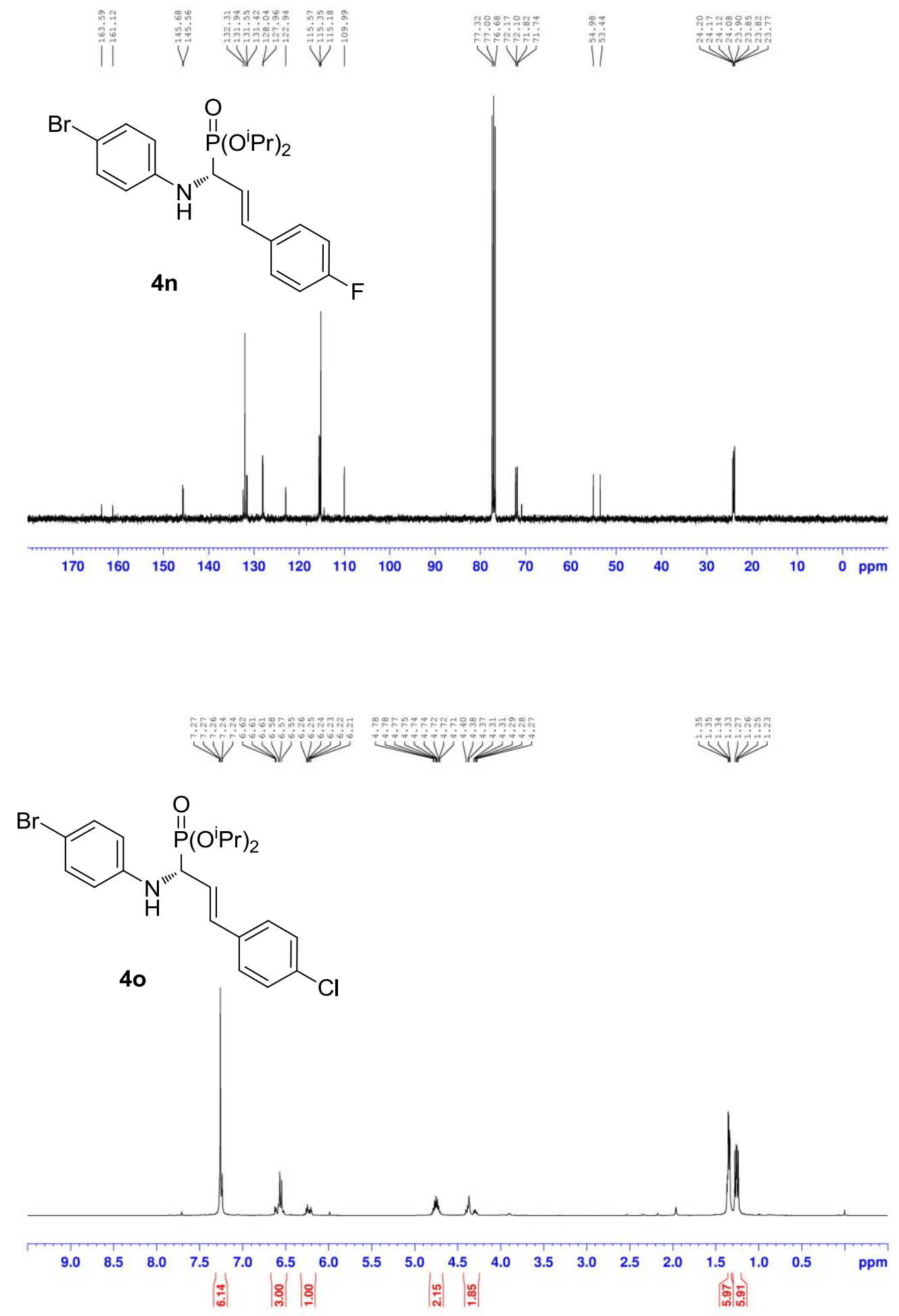

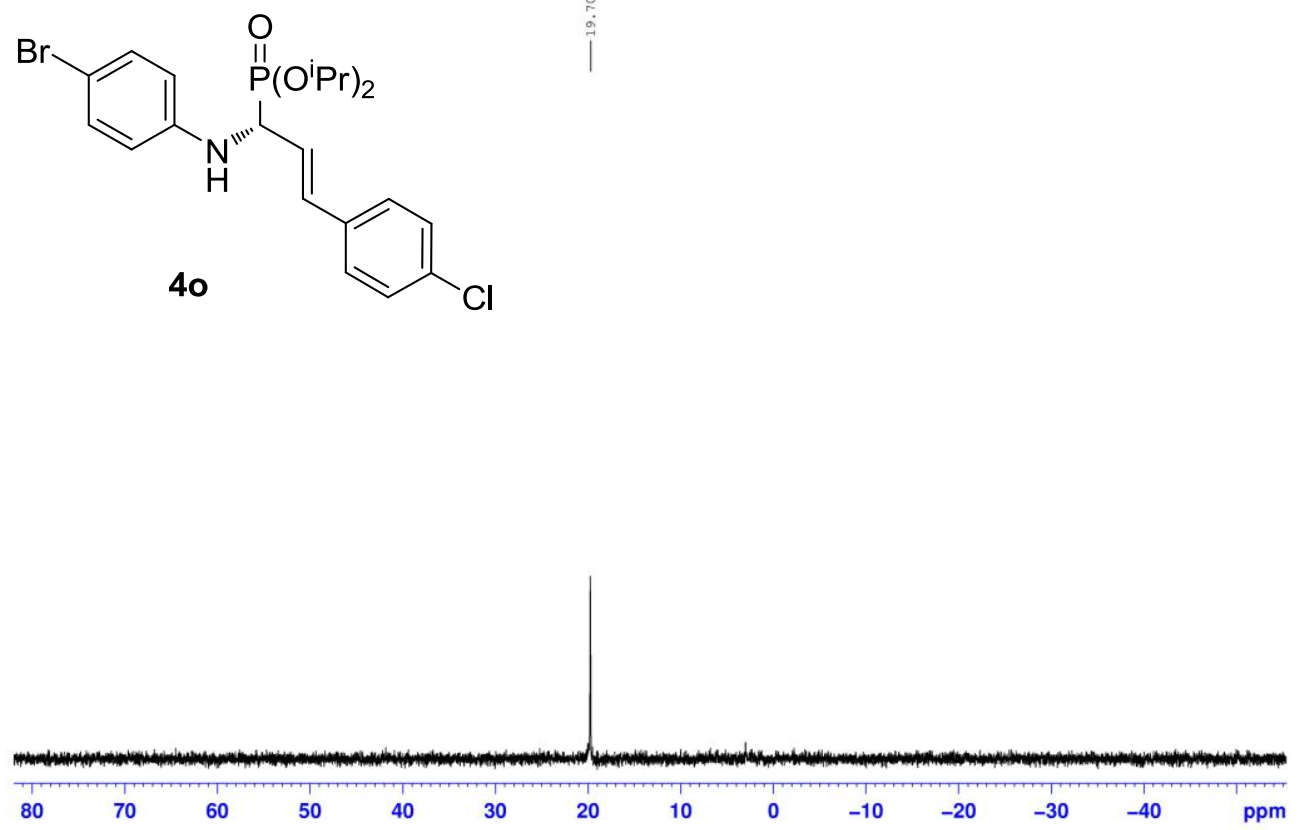

V
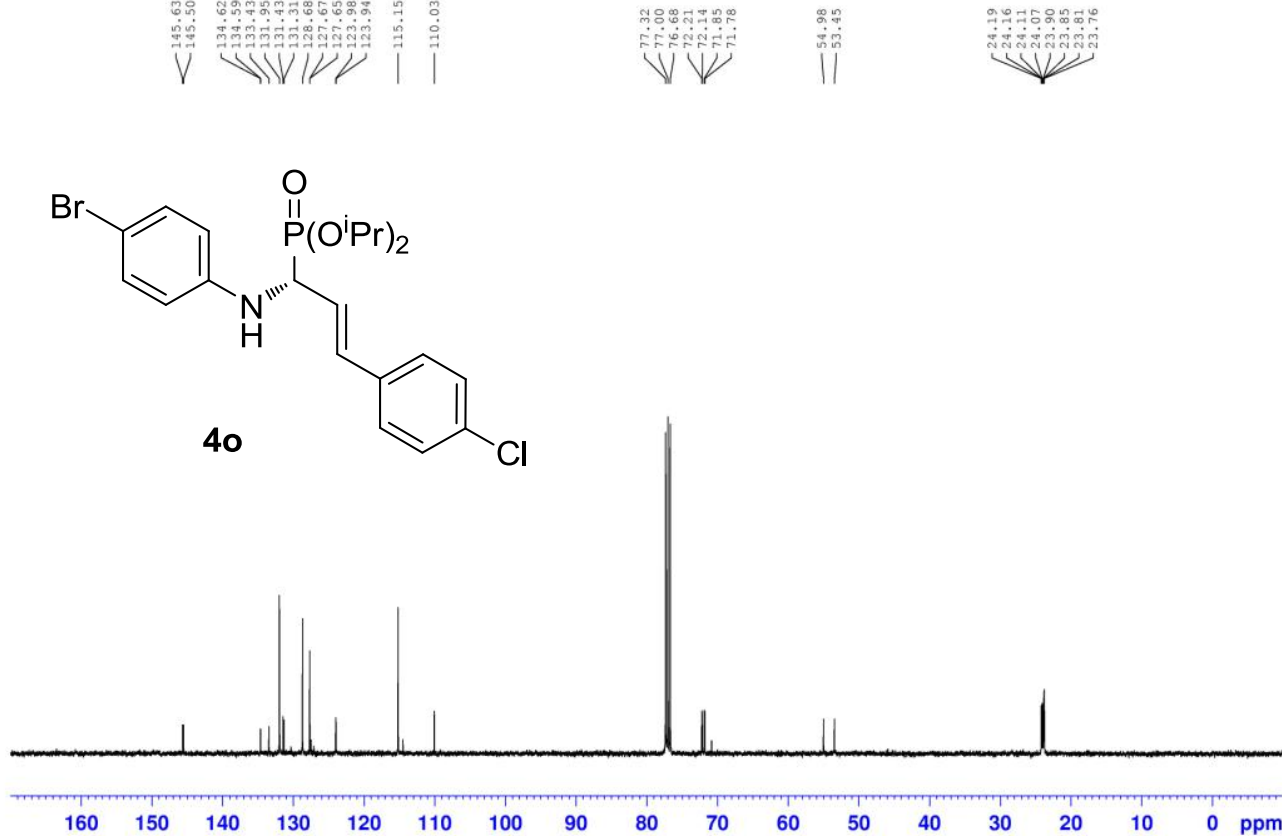

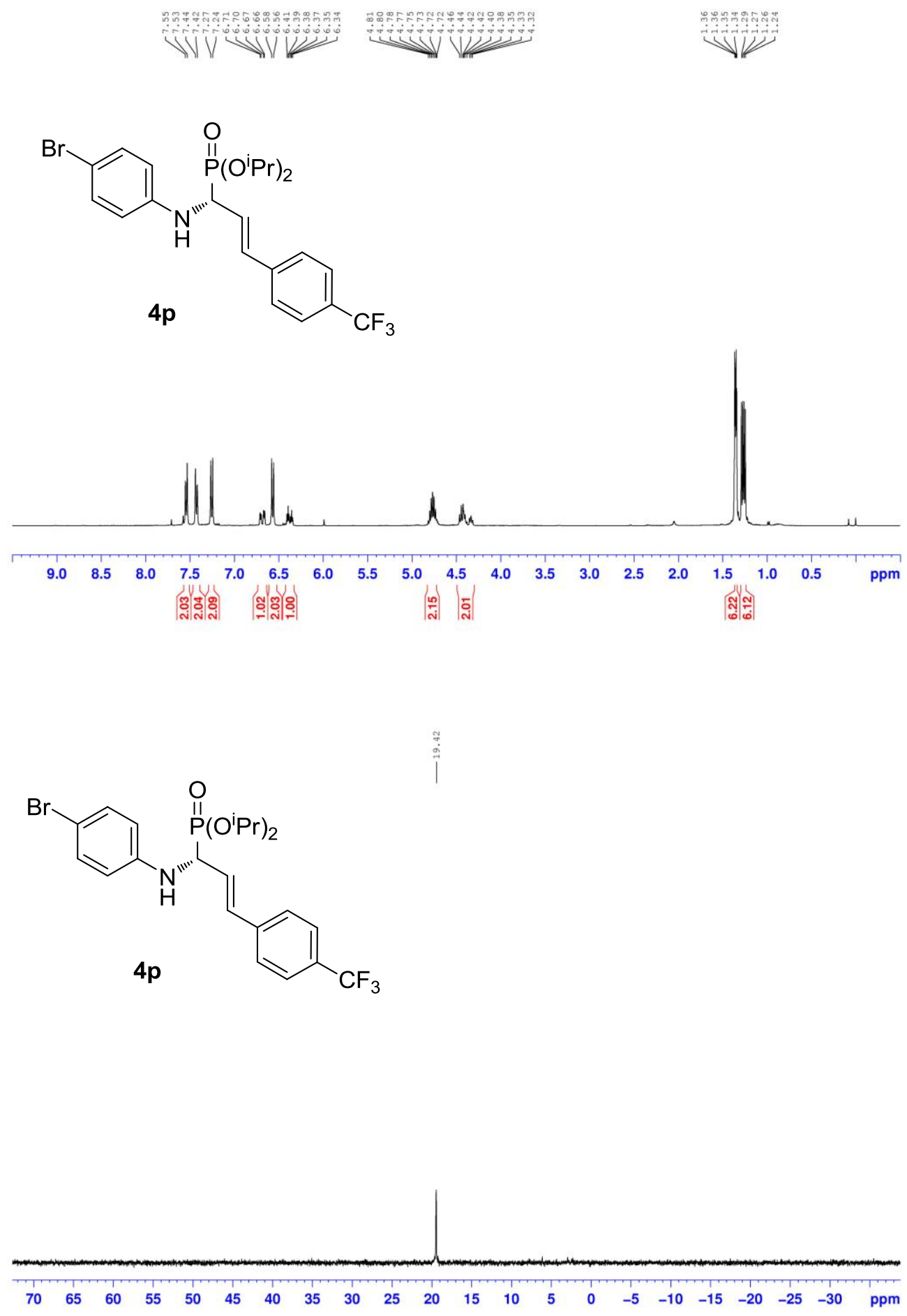

S-52 
<smiles>O=[PH+](O)C(/C=C/c1ccc(C(F)(F)F)cc1)[NH+](c1ccccc1)c1ccc(Br)cc1</smiles>
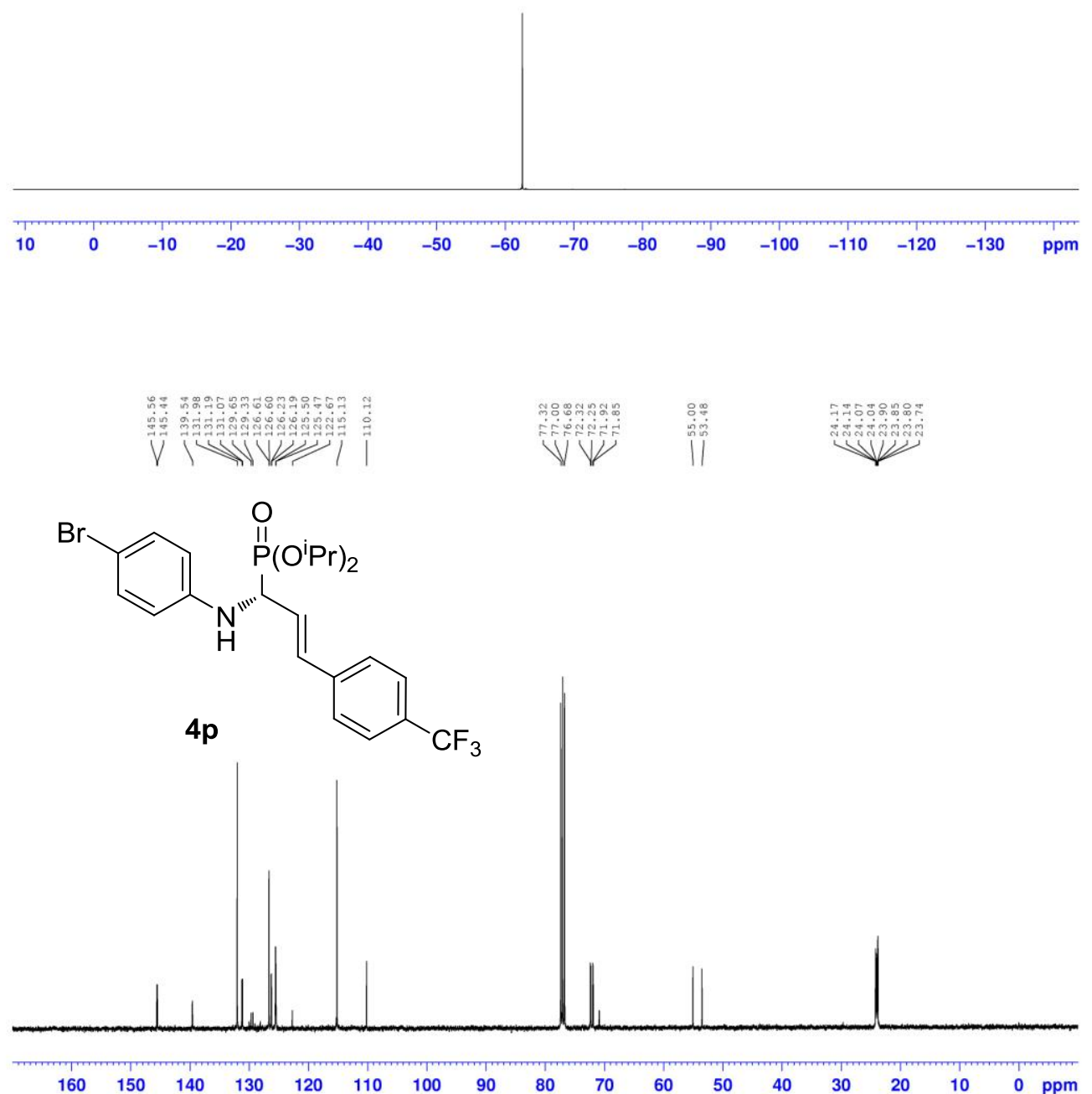
<smiles>O=[PH+](O)C(/C=C/c1ccc(Br)cc1F)[NH+]([O-])c1ccc(Br)cc1</smiles>

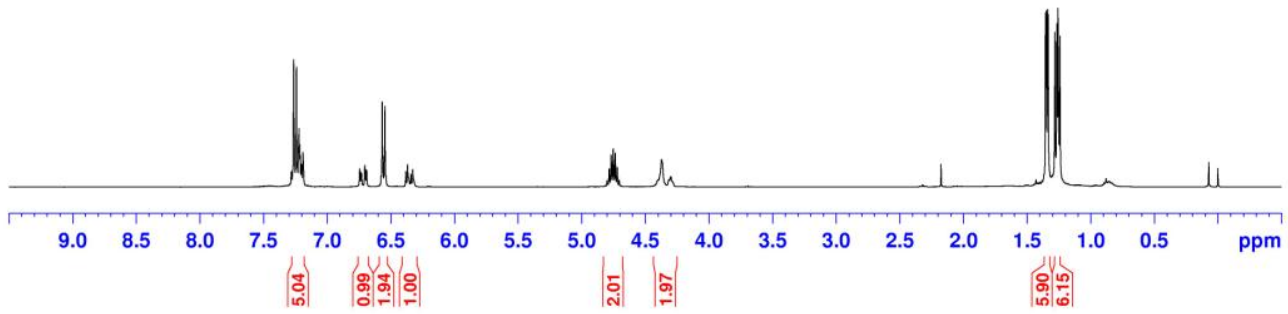<smiles>O=[PH](OCc1ccccc1)[C@H](/C=C/c1ccc(Br)cc1F)Nc1ccc(Br)cc1</smiles>

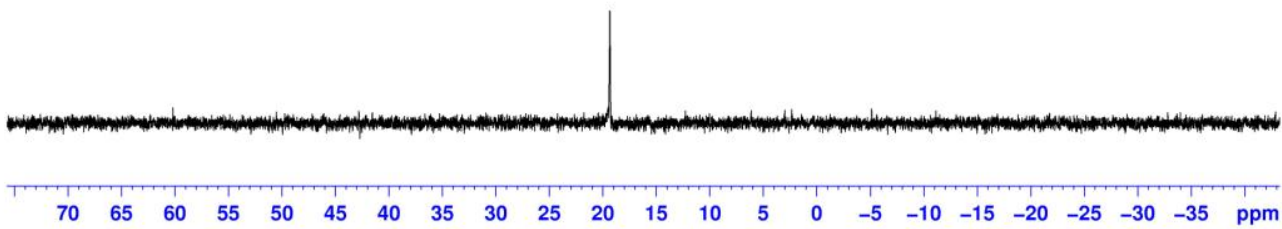


<smiles>O=[P+](O)C(/C=C/c1ccc(Br)cc1F)[NH+](c1ccccc1)c1ccc(Br)cc1</smiles>
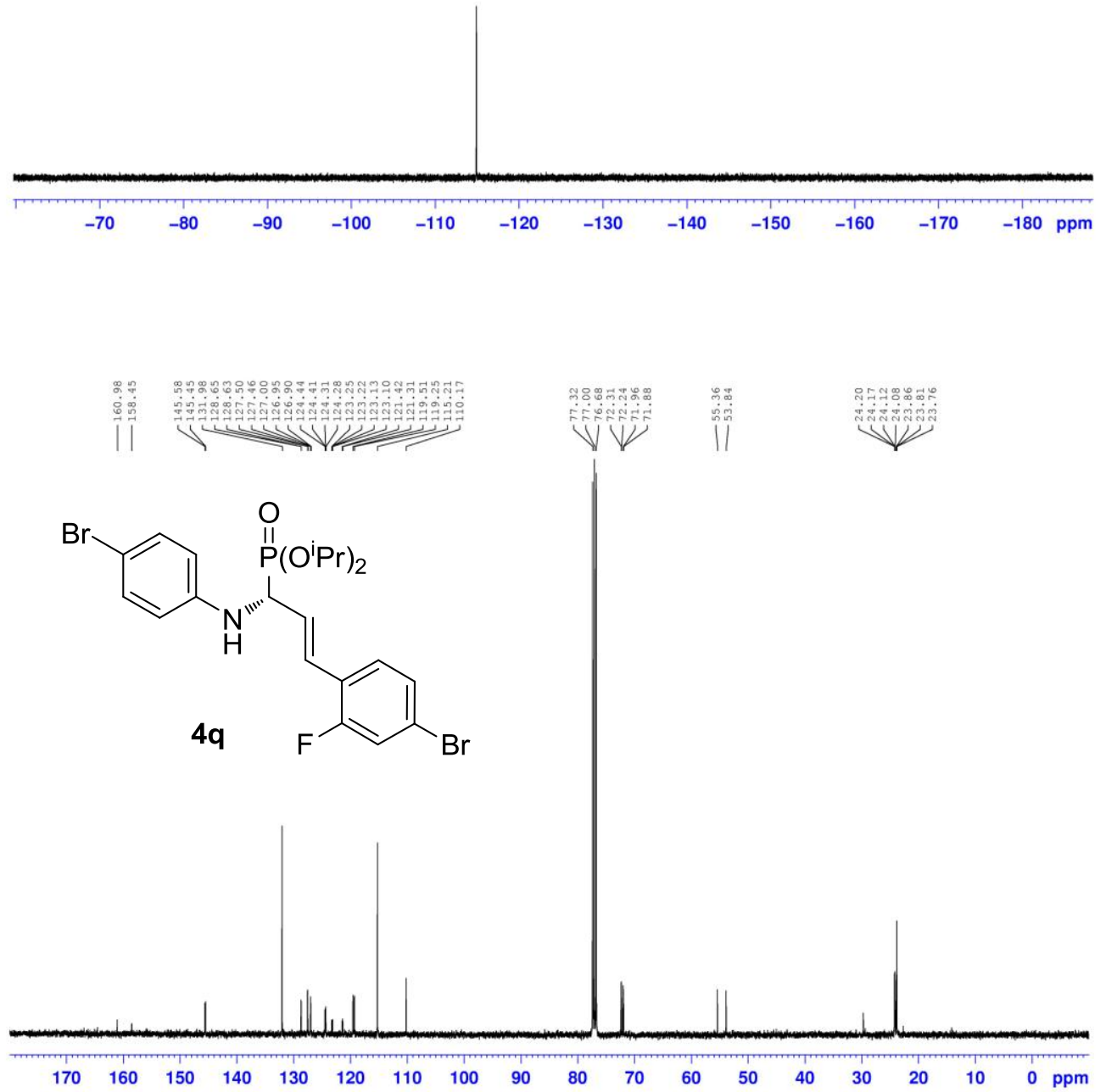
<smiles>O=P[C@@H](Nc1ccc(Br)cc1)C(/C=C/c1cc(Br)ccc1F)[PH+]=O</smiles>

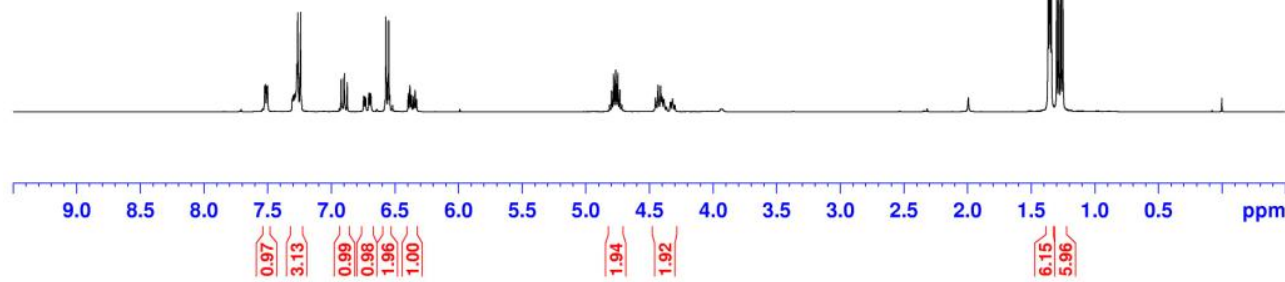<smiles>O=[PH+]C(/C=C/c1cc(Br)ccc1F)[NH+]([O+])c1ccc(Br)cc1</smiles>

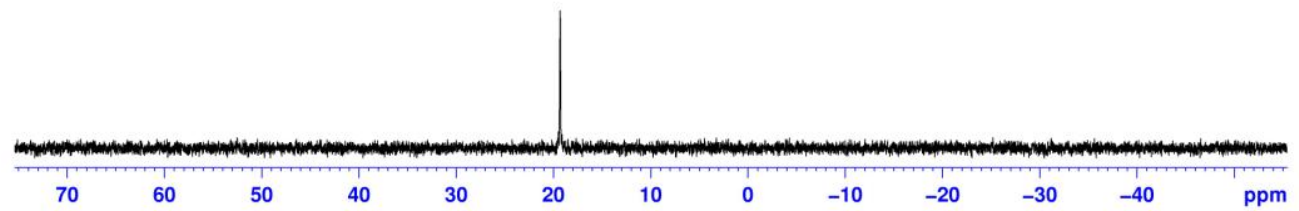


<smiles>O=[P+](O)C(/C=C/c1cc(Br)ccc1F)[NH+](c1ccccc1)c1ccc(Br)cc1</smiles>
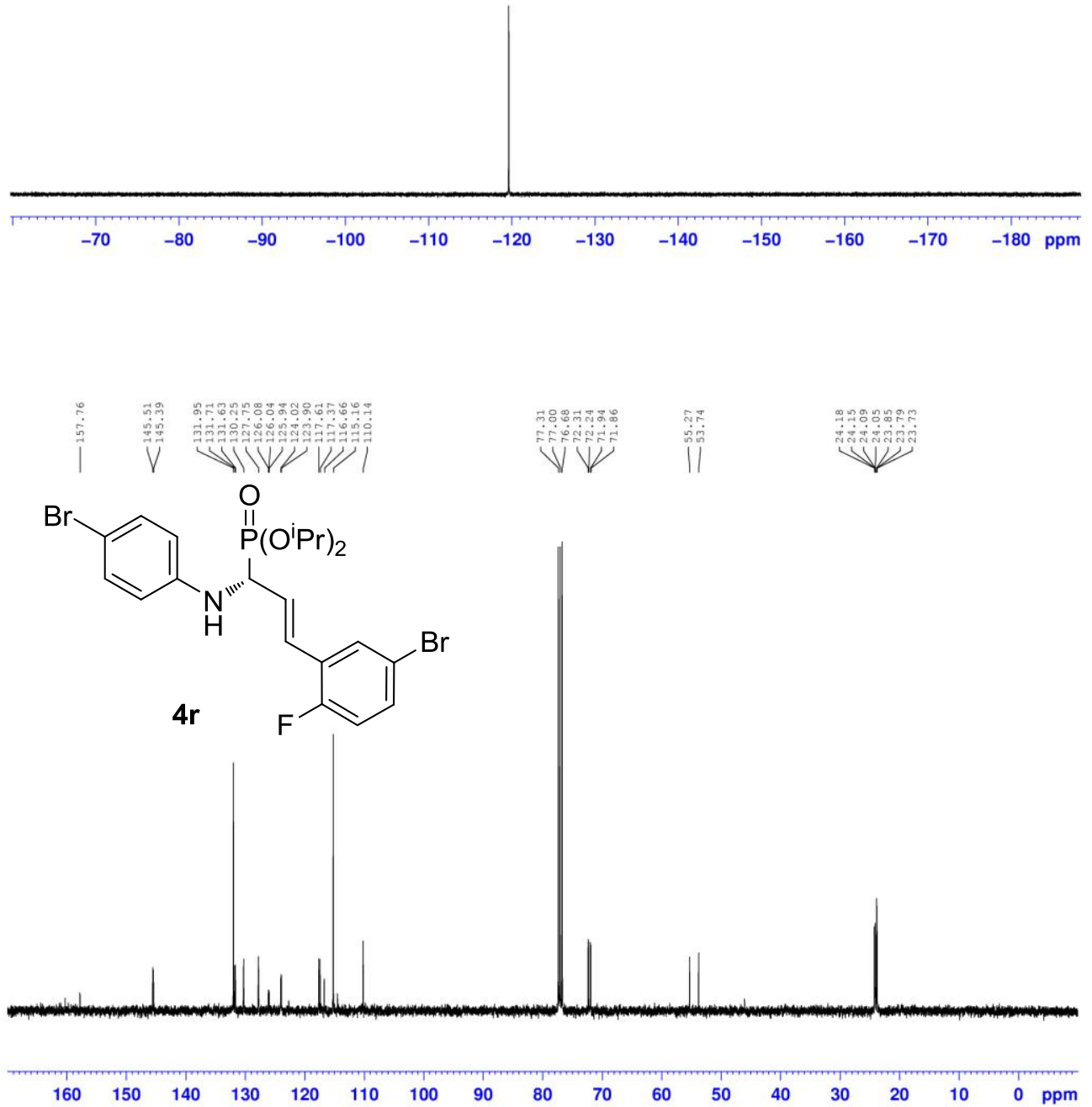
<smiles>CS(=O)(=O)OC(=O)[C@H](/C=C/c1ccc2ccccc2c1)Nc1ccc(Br)cc1</smiles>

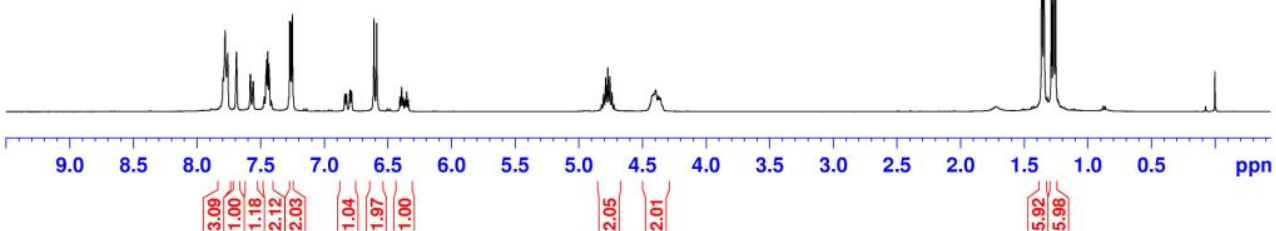<smiles>O=[PH+]([O-])C(/C=C/c1ccc2ccccc2c1)[NH+]([O-])c1ccc(Br)cc1</smiles>

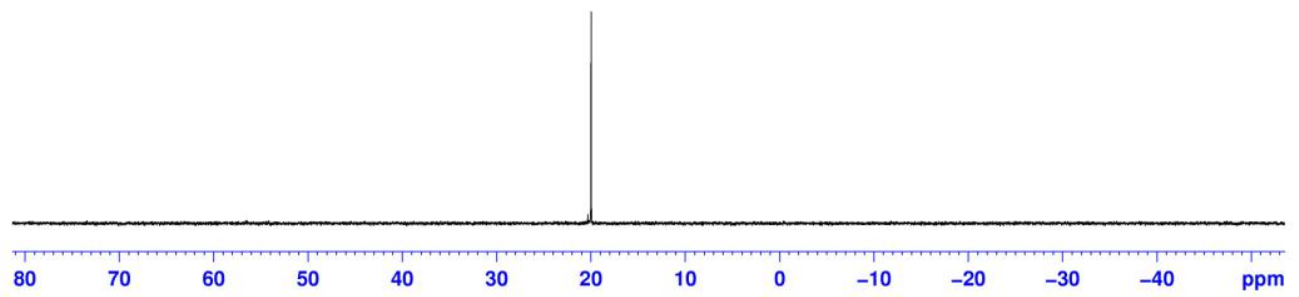




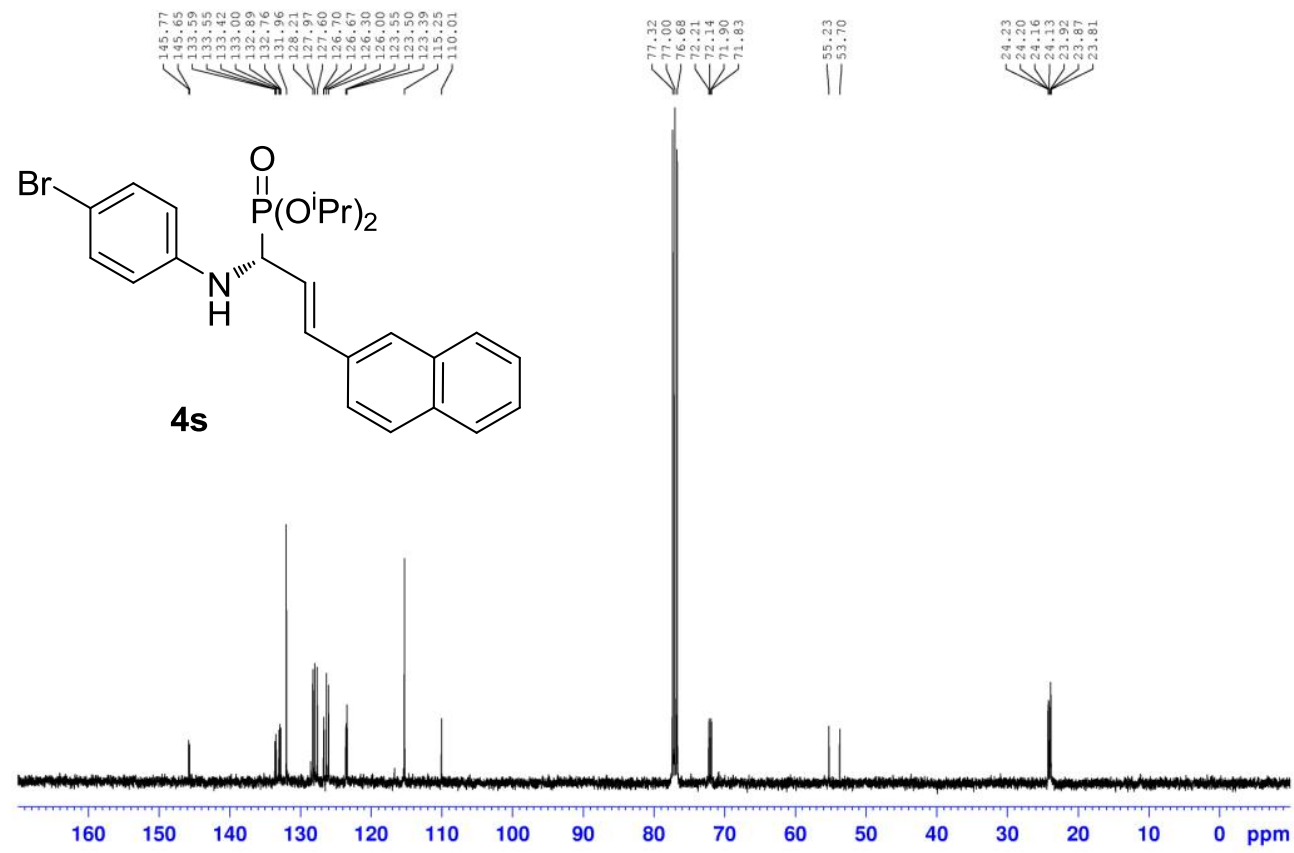

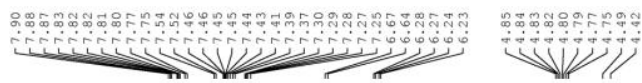
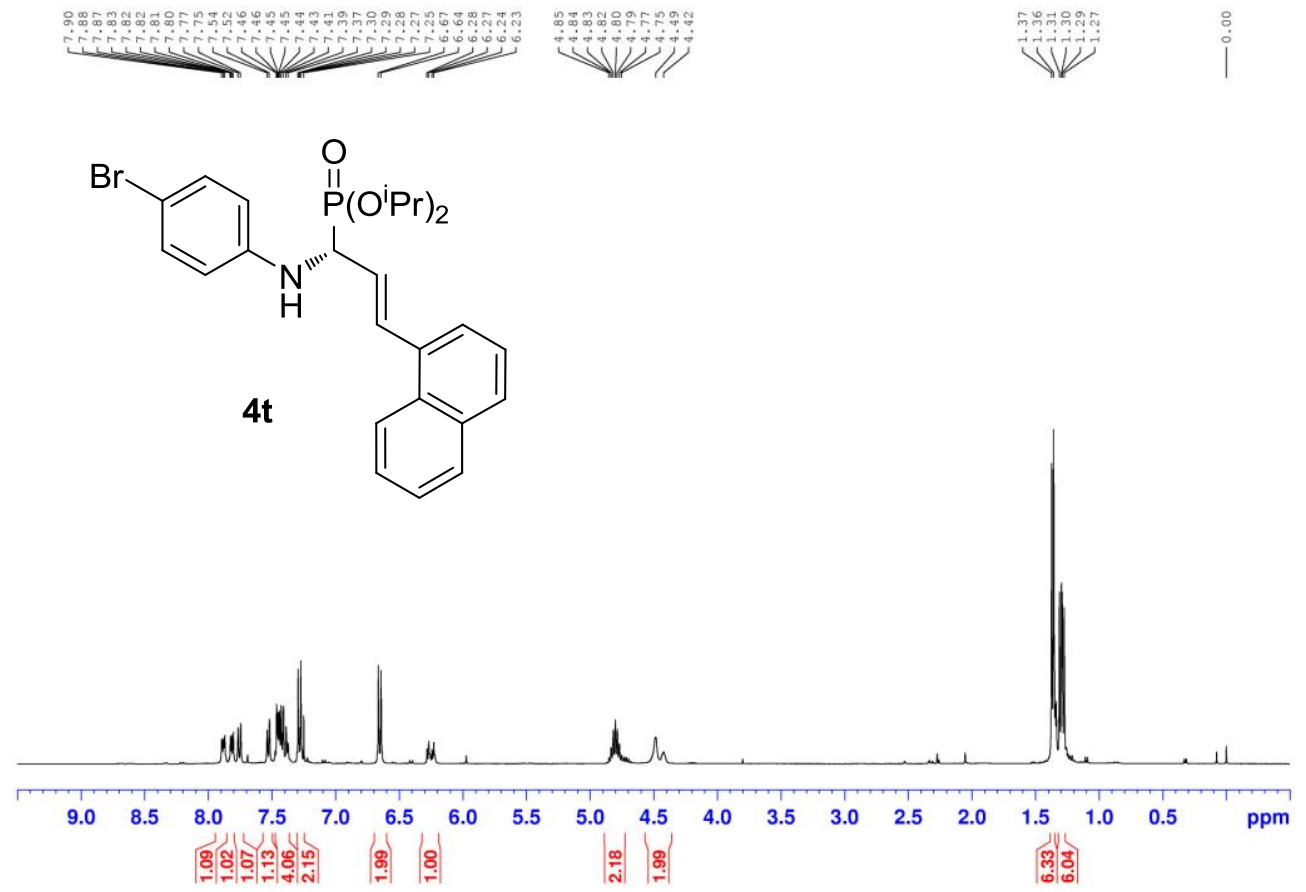
<smiles>O=P[PH2+]([OH2+])[C@H](/C=C/c1cccc2ccccc12)Nc1ccc(Br)cc1</smiles>
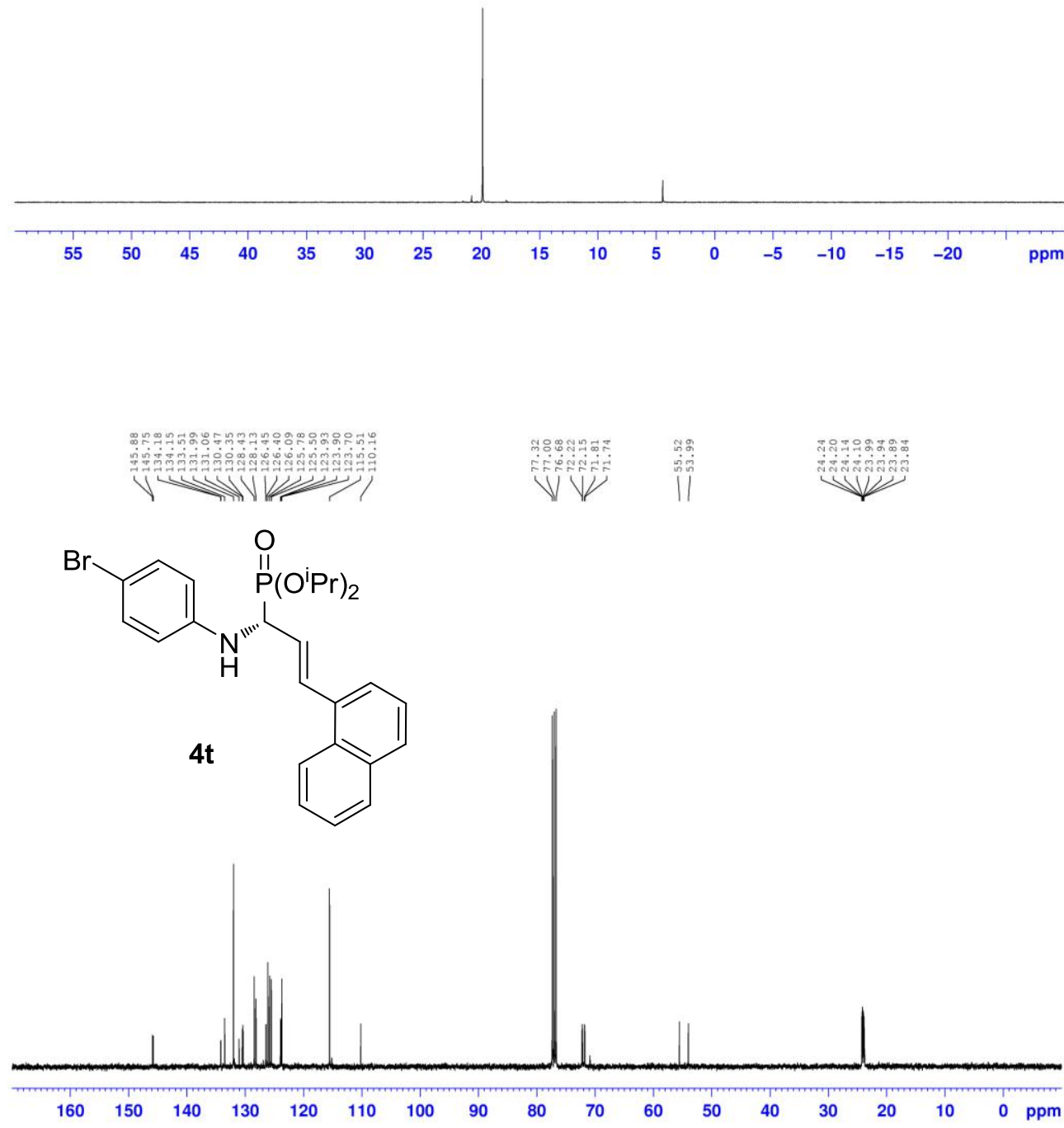


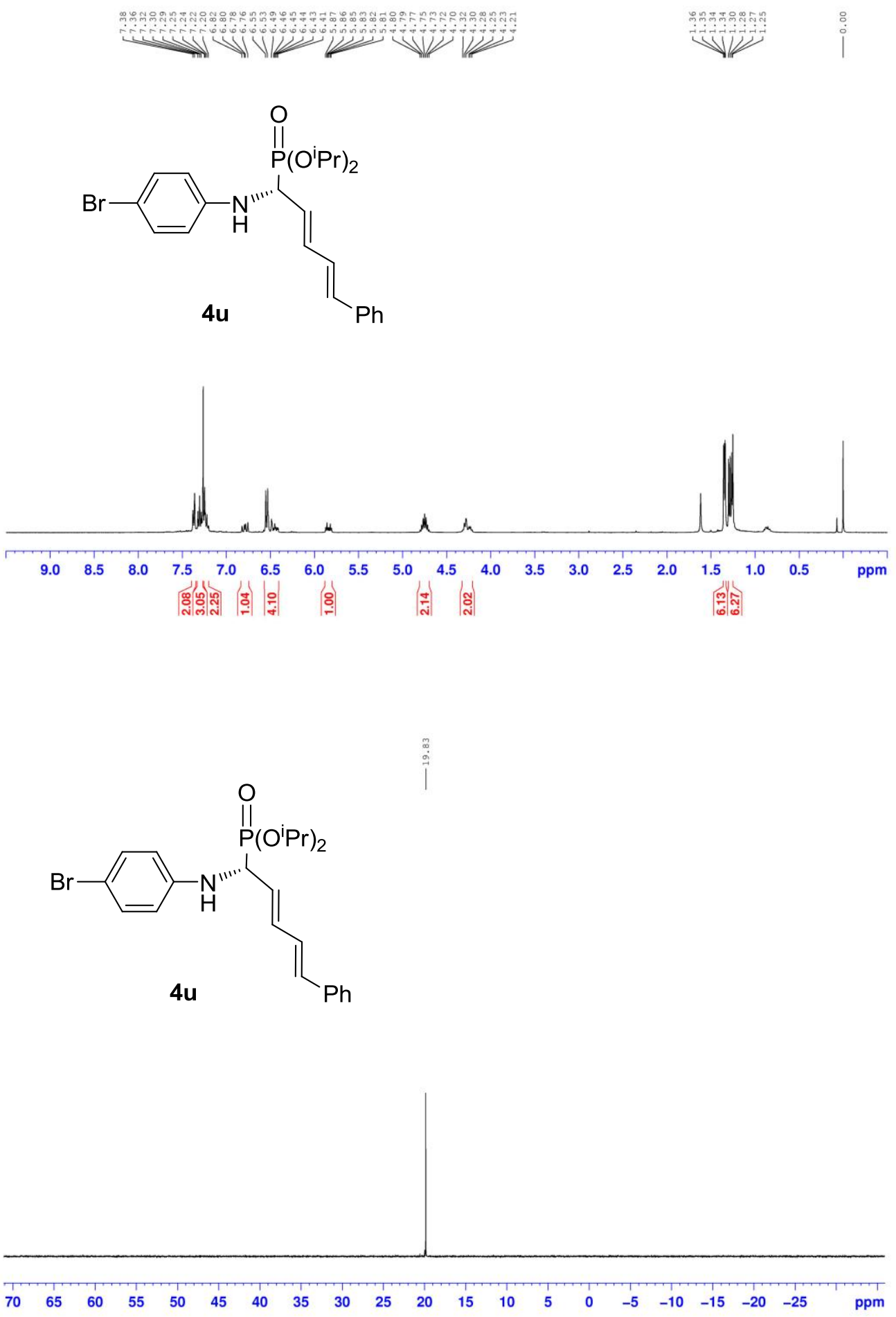



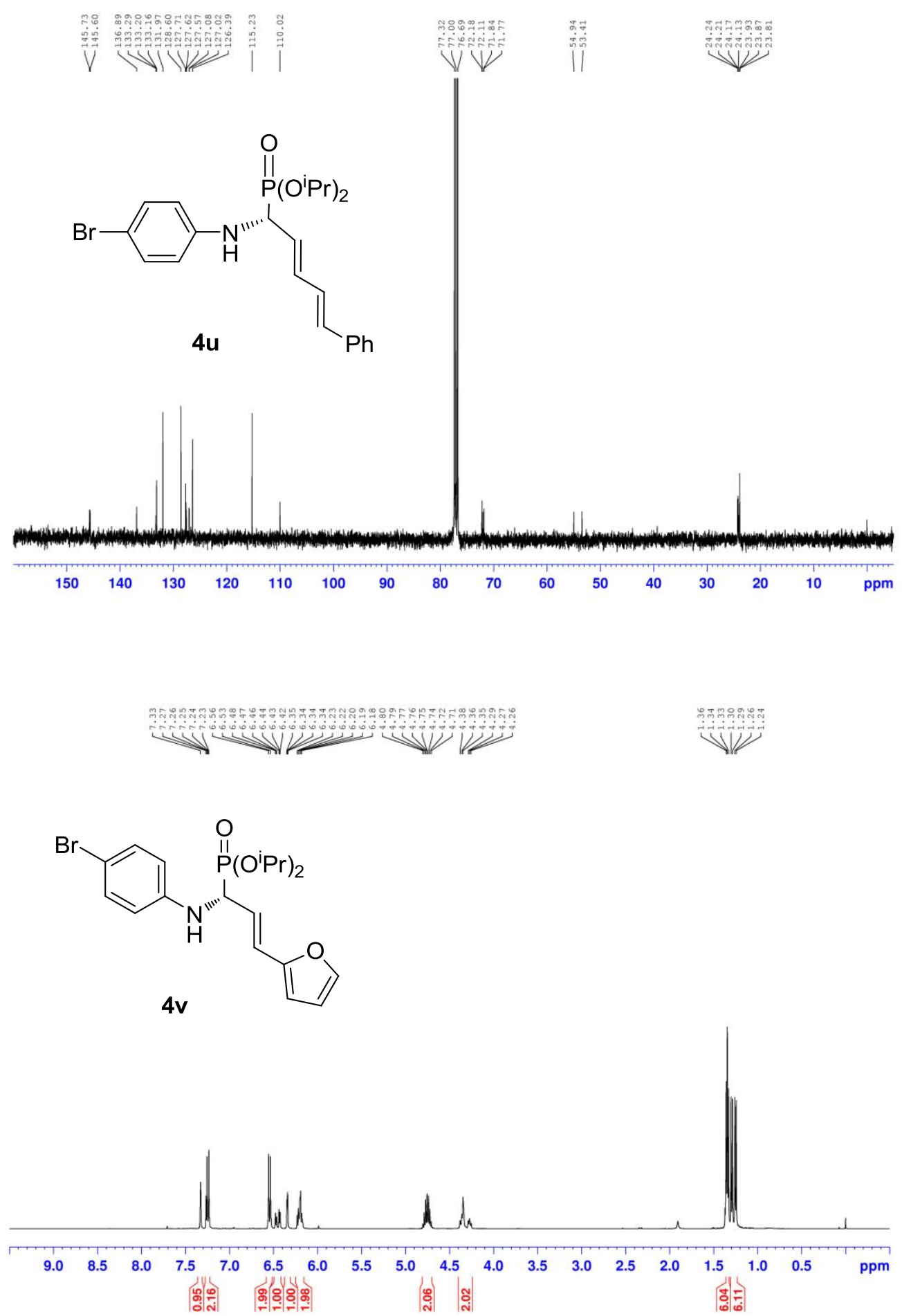

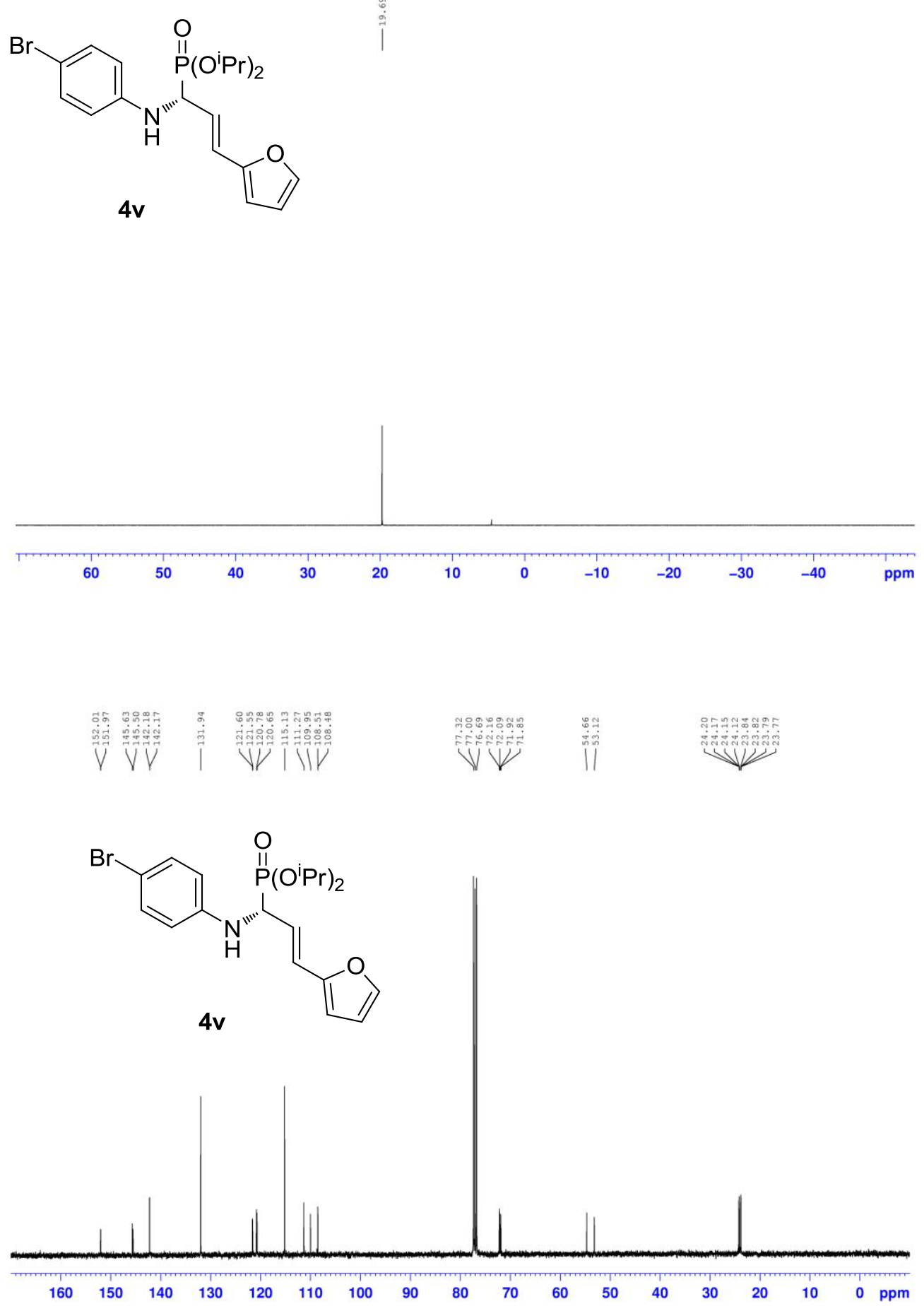

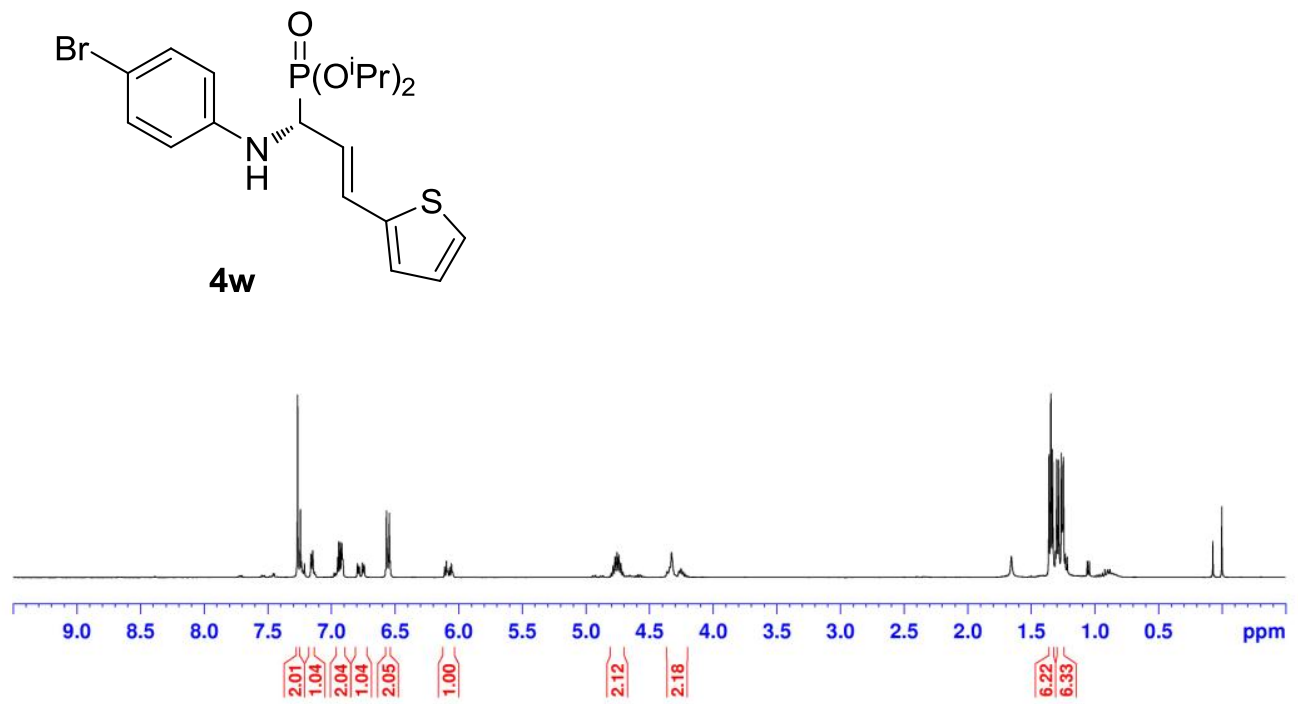

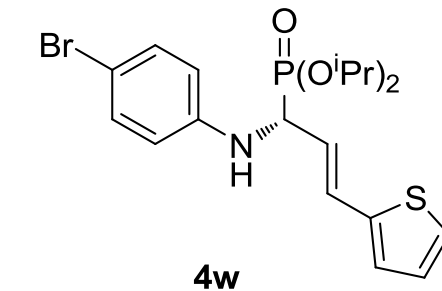

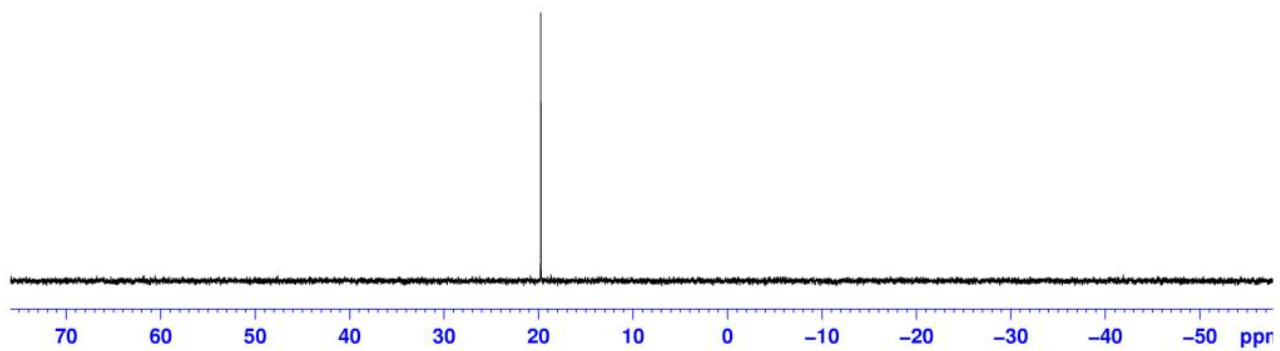



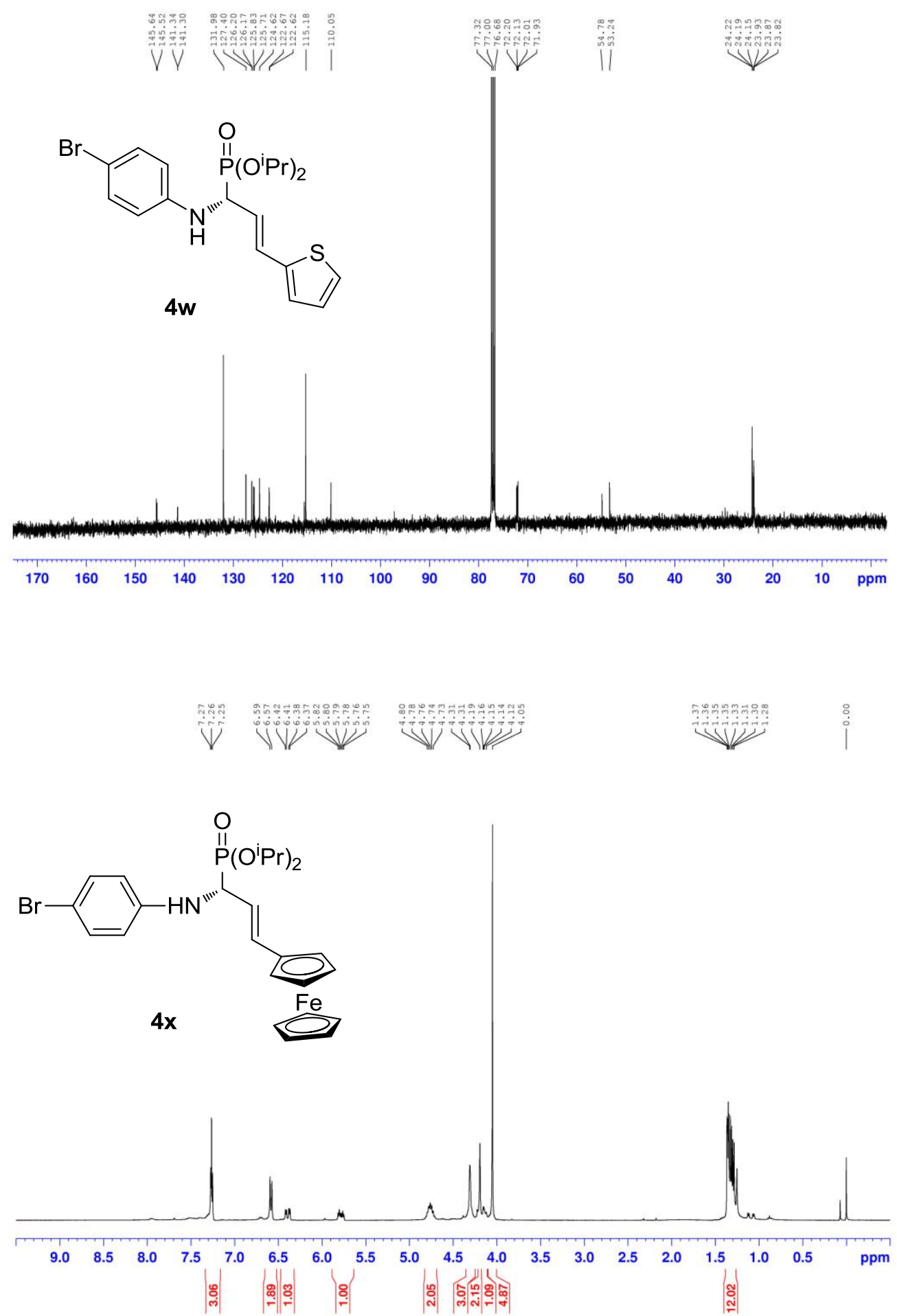

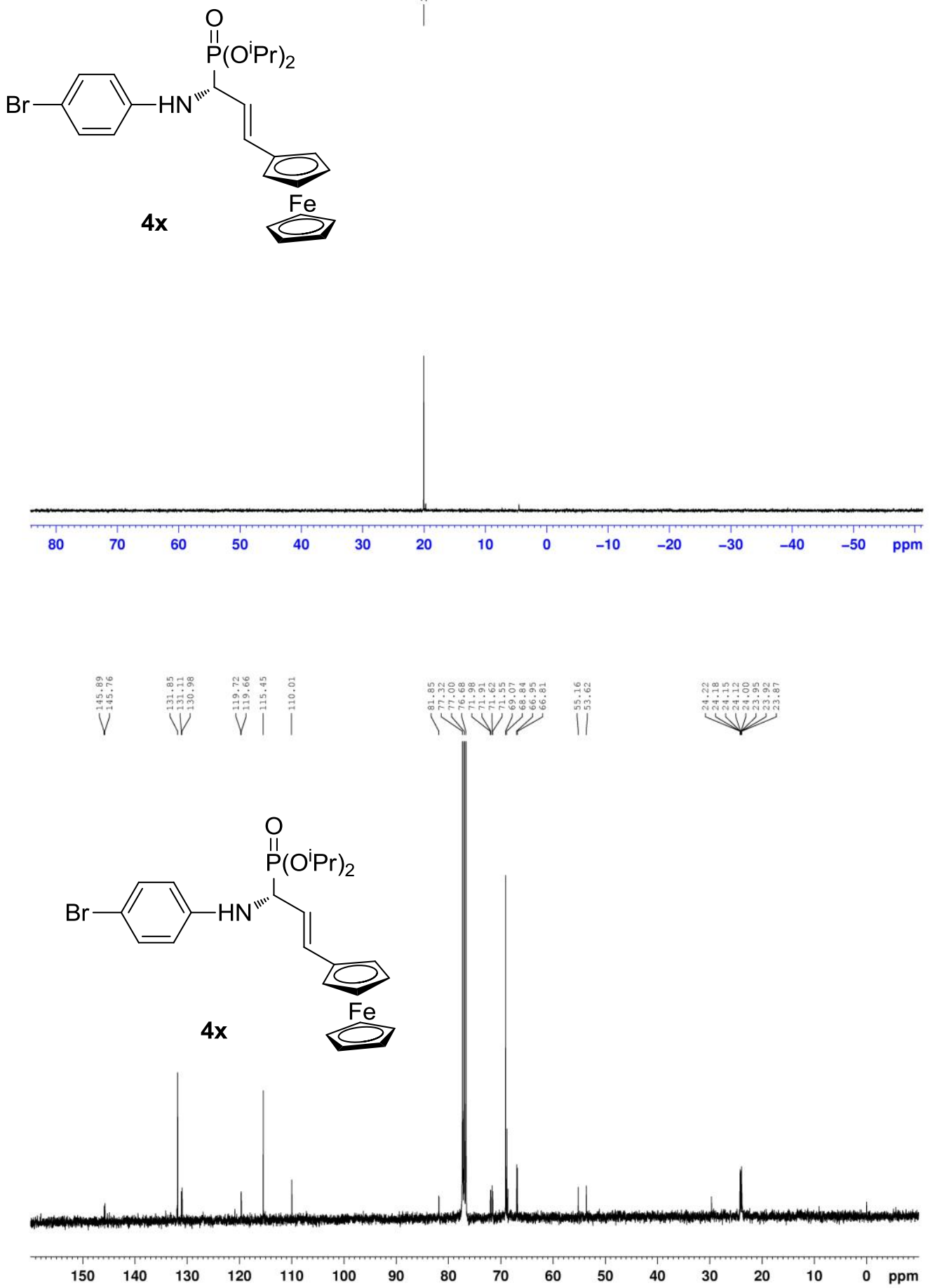
<smiles>N[C@@H](/C=C/c1ccccc1)P(=O)=O</smiles>

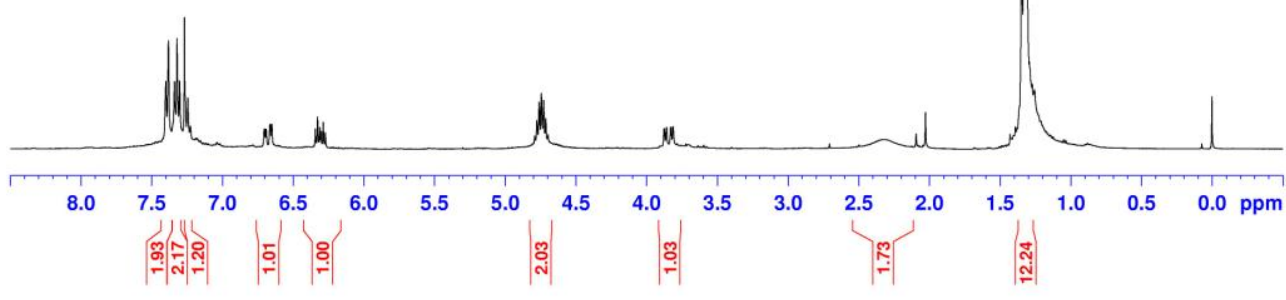<smiles>N[C@@H](/C=C/c1ccccc1)[P+](=O)O</smiles>

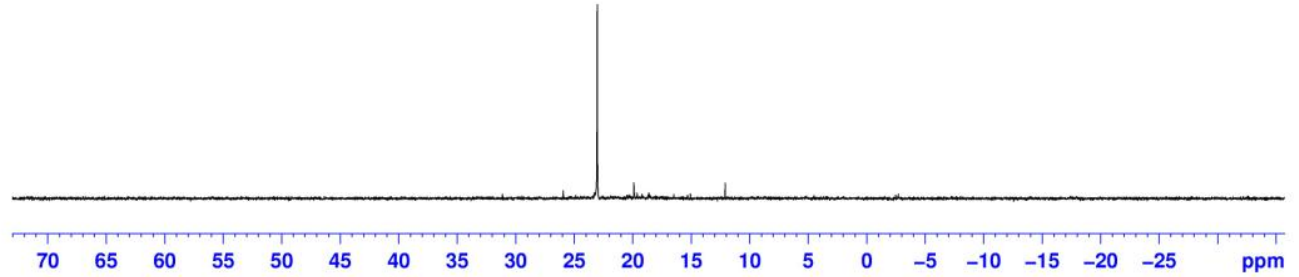




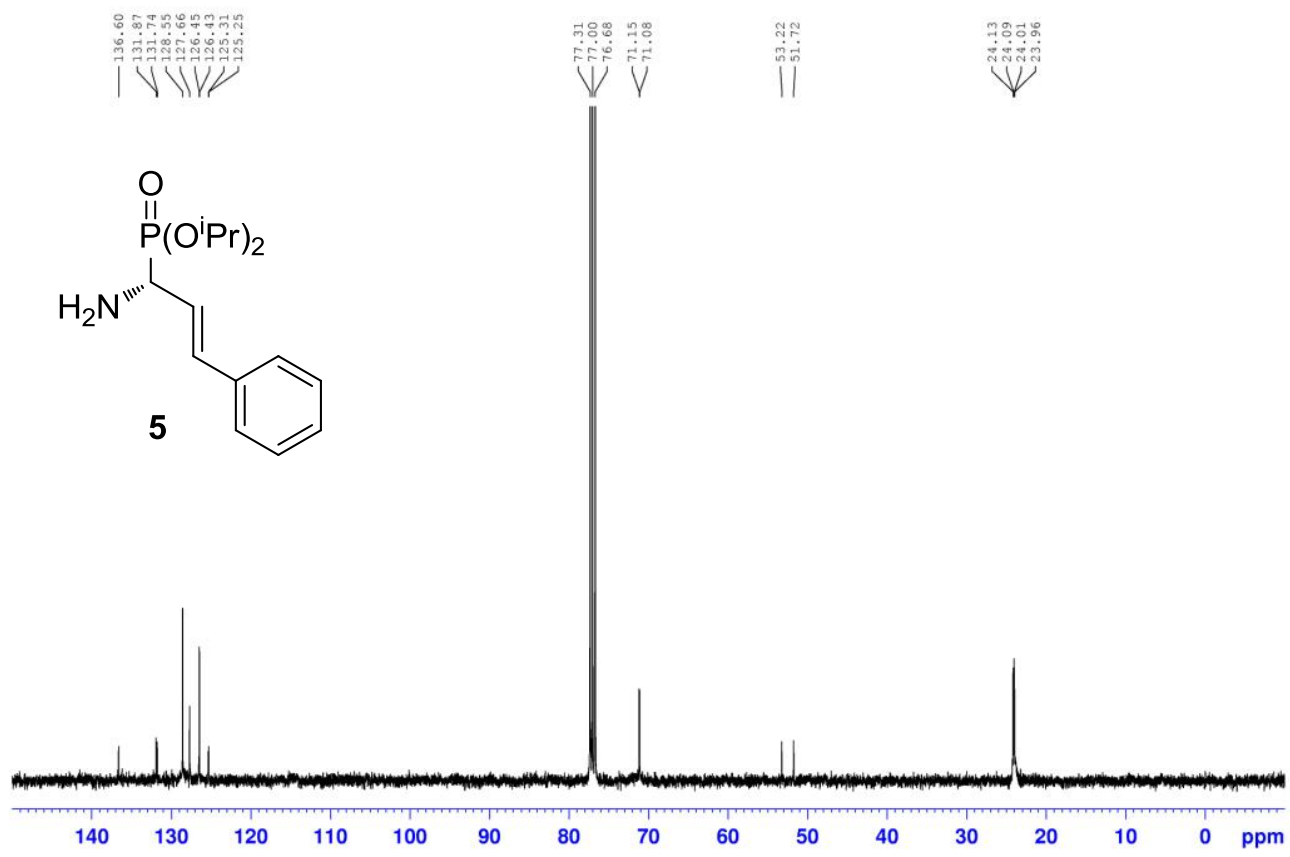

